\title{
The Chandra Deep Field North Survey. V. 1 Ms Source Catalogs
}

\author{
W.N. Brandt, ${ }^{1}$ D.M. Alexander, ${ }^{1}$ A.E. Hornschemeier, ${ }^{1}$ G.P. Garmire,${ }^{1}$ D.P. Schneider, ${ }^{1}$ \\ A.J. Barger,${ }^{2,3,4}$ F.E. Bauer, ${ }^{1}$ P.S. Broos,${ }^{1}$ L.L. Cowie,${ }^{2}$ L.K. Townsley, ${ }^{1}$ D.N. Burrows, ${ }^{1}$ \\ G. Chartas, ${ }^{1}$ E.D. Feigelson, ${ }^{1}$ R.E. Griffiths,${ }^{5}$ J.A. Nousek, ${ }^{1}$ and W.L.W. Sargent ${ }^{6}$
}

\begin{abstract}
An extremely deep X-ray survey $(\approx 1 \mathrm{Ms})$ of the Hubble Deep Field North and its environs $\left(\approx 450 \operatorname{arcmin}^{2}\right)$ has been performed with the Advanced CCD Imaging Spectrometer on board the Chandra X-ray Observatory. This is one of the two deepest X-ray surveys ever performed; for point sources near the aim point it reaches $0.5-2.0 \mathrm{keV}$ and 2-8 keV flux limits of $\approx 3 \times 10^{-17} \mathrm{erg} \mathrm{cm}^{-2} \mathrm{~s}^{-1}$ and $\approx 2 \times 10^{-16} \mathrm{erg} \mathrm{cm}^{-2} \mathrm{~s}^{-1}$, respectively. Here we provide source catalogs along with details of the observations, data reduction, and technical analysis. Observing conditions, such as background, were excellent for almost all of the exposure.

We have detected 370 distinct point sources: 360 in the $0.5-8.0 \mathrm{keV}$ band, 325 in the $0.5-2.0 \mathrm{keV}$ band, 265 in the $2-8 \mathrm{keV}$ band, and 145 in the $4-8 \mathrm{keV}$ band. Two new Chandra sources in the HDF-N itself are reported and discussed. Source positions are accurate to within $0.6-1.7^{\prime \prime}$ (at $\approx 90 \%$ confidence) depending mainly on the off-axis angle. We also detect two highly significant extended X-ray sources and several other likely extended X-ray sources.

We present basic number count results for sources located near the center of the field. Source densities of $7100_{-940}^{+1100} \mathrm{deg}^{-2}\left(\right.$ at $4.2 \times 10^{-17} \mathrm{erg} \mathrm{cm}^{-2} \mathrm{~s}^{-1}$ ) and $4200_{-580}^{+670} \mathrm{deg}^{-2}$ (at $3.8 \times 10^{-16} \mathrm{erg} \mathrm{cm}^{-2} \mathrm{~s}^{-1}$ ) are observed in the soft and hard bands, respectively.

Subject headings: diffuse radiation - surveys - cosmology: observations - galaxies: active - X-rays: galaxies - X-rays: general.

\footnotetext{
${ }^{1}$ Department of Astronomy \& Astrophysics, 525 Davey Laboratory, The Pennsylvania State University, University Park, PA 16802

${ }^{2}$ Institute for Astronomy, University of Hawaii, 2680 Woodlawn Drive, Honolulu, HI 96822

${ }^{3}$ Department of Astronomy, University of Wisconsin-Madison, 475 N. Charter Street, Madison, WI 53706

${ }^{4}$ Hubble Fellow and Chandra Fellow at Large

${ }^{5}$ Department of Physics, Carnegie Mellon University, Pittsburgh, PA 15213

${ }^{6}$ Palomar Observatory, California Institute of Technology, Pasadena, CA 91125
} 


\section{Introduction}

We have performed a deep X-ray survey $(\approx 1 \mathrm{Ms})$ of the Hubble Deep Field North (HDF-N; Williams et al. 1996, hereafter W96; Ferguson, Dickinson, \& Williams 2000) and its environs with the Chandra X-ray Observatory (hereafter Chandra): the Chandra Deep Field North (hereafter CDF-N) survey. This is one of the two deepest X-ray surveys ever conducted, the other being the Chandra Deep Field South survey (e.g., Tozzi et al. 2001; P. Rosati et al., in preparation). Near the aim point, the CDF-N observation reaches $0.5-2.0 \mathrm{keV}$ and $2-8 \mathrm{keV}$ limiting fluxes of $\approx 3 \times 10^{-17} \mathrm{erg} \mathrm{cm}^{-2} \mathrm{~s}^{-1}$ and $\approx 2 \times 10^{-16} \mathrm{erg} \mathrm{cm}^{-2} \mathrm{~s}^{-1}$, respectively; these flux limits are $\approx 40$ and $\approx 400$ times fainter than achieved by pre-Chandra missions. In Figure 1 we compare the 0.5-2.0 keV flux limit and solid angle of this survey to those of several other extragalactic X-ray surveys. This survey has both the sensitivity and positional accuracy needed to complement the deepest surveys at other wavelengths, and much of the $\approx 450 \operatorname{arcmin}^{2}$ area surveyed has extensive radio, submillimeter, infrared, and optical coverage (see Livio, Fall, \& Madau 1998 and Ferguson et al. 2000 for reviews).

The main goals of the CDF-N survey are (1) to understand the broad-band emission and nature of the sources producing the X-ray background in the $0.5-8.0 \mathrm{keV}$ band, and (2) to investigate the $\mathrm{X}$-ray emission properties of physically interesting sources identified at other wavelengths. Thus far, we have presented results for the HDF-N (Hornschemeier et al. 2000, hereafter Paper I; Brandt et al. 2001a, Paper IV) as well as for larger fields centered on the HDF-N (Hornschemeier et al. 2001, Paper II; Garmire et al. 2001, Paper III; Barger et al. 2001). Due to the fact that the Chandra observations were performed in an incremental manner from 1999 November to 2001 March, the studies above were all performed with less than the full $\approx 1$ Ms exposure.

In this paper, we provide source catalogs derived from the $\approx 1 \mathrm{Ms}$ data set along with details of the observations, data reduction, and technical analysis. Our intention is to provide this information to the community in as timely a manner as possible. We have deliberately avoided follow-up investigations and detailed scientific interpretation in this paper; these will be presented in subsequent papers (and many such results have been presented in the papers cited above). For example, companion papers by Alexander et al. (2001, Paper VI), Brandt et al. (2001b, Paper VII), A.J. Barger et al., in preparation, and F.E. Bauer et al., in preparation present results on

optically faint X-ray sources, X-ray emission from Lyman break galaxies, optical follow-up imaging and spectroscopy, and extended X-ray sources.

In $\S 2$ we describe the observations and data reduction. In $\S 3$ we describe the data analysis and results, with emphasis on the detection of point sources (§3.2) and extended sources ( $\S 3.3)$. We also present basic number count results for point sources in $\S 3.2$. Our conclusions and summary are presented in $\S 4$.

The Galactic column density along this line of sight is $(1.6 \pm 0.4) \times 10^{20} \mathrm{~cm}^{-2}$ (Stark et al. 1992). $H_{0}=70 \mathrm{~km} \mathrm{~s}^{-1} \mathrm{Mpc}^{-1}, \Omega_{\mathrm{M}}=1 / 3$, and $\Omega_{\Lambda}=2 / 3$ are adopted throughout this paper. Coordinates throughout this paper are J2000. The HDF-N itself is centered at $\alpha_{2000}=12^{\mathrm{h}} 36^{\mathrm{m}}$ 
$49^{\text {s }} 4, \delta_{2000}=+62^{\circ} 12^{\prime} 58^{\prime \prime}$, corresponding to $l=125.888, b=54^{\circ} 828$ (W96).

\section{Observations and Data Reduction}

\subsection{Instrumentation}

All observations of the CDF-N field were performed with the Advanced CCD Imaging Spectrometer (ACIS; G.P. Garmire et al., in preparation) on Chandra (Weisskopf et al. 2000). ${ }^{1}$ ACIS consists of ten CCDs designed for efficient X-ray detection and spectroscopy. Four of the CCDs (ACIS-I; CCDs I0-I3) are arranged in a $2 \times 2$ array with each CCD tipped slightly to approximate the curved focal surface of the Chandra High Resolution Mirror Assembly (HRMA). The remaining six CCDs (ACIS-S; CCDs S0-S5) are set in a linear array and are tipped to approximate the Rowland circle of the objective gratings that can be inserted behind the HRMA. The CCD which lies on-axis in ACIS-I is I3, and the full ACIS-I field of view is $16.9 \times 16^{\prime} .9$.

The ACIS pixel size is $\approx 0$ "' 492 . The $95 \%$ encircled-energy radius for an energy of $1.5 \mathrm{keV}$ at the aim point is $\approx 1^{\prime \prime} .8$. This radius increases to $\approx 2$.' 7 when $4^{\prime}$ from the aim point and $\approx 7^{\prime \prime} .5$ when $8^{\prime}$ from the aim point (Feigelson, Broos, \& Gaffney 2000; Jerius et al. 2000; M. Karovska and P. Zhao 2001, private communication). ${ }^{2}$ Note that at higher energies the aim-point PSF is significantly broader than at $1.5 \mathrm{keV}$.

\subsection{Observational Parameters and Conditions}

The CDF-N was observed in twelve separate observations as detailed in Table 1 . The total exposure time was $975.3 \mathrm{ks}$. The HDF-N was placed near the aim point of the ACIS-I array during all observations, and care was taken to keep the HDF-N away from the gaps between the CCDs. The focal-plane temperature, which governs several characteristics of the CCD behavior, in particular the Charge Transfer Inefficiency (CTI), was $-110^{\circ} \mathrm{C}$ during the first three observations and $-120^{\circ} \mathrm{C}$ during the others. When observing with ACIS-I, two CCDs from ACIS-S, typically S2 and S3, can be operated. CCD S3 was turned off during the HDF-N observations due to the higher background level of this device; this property could cause telemetry saturation during background flares. CCD S2, however, was operated.

The region covered by the Chandra observations is considerably larger than $16.9 \times 16$ '.9 due to the different observation pointings and roll angles (see Table 1). These variations were necessary to satisfy the roll constraints of Chandra while keeping the HDF-N itself near the aim point and away

\footnotetext{
${ }^{1}$ For additional information on the ACIS and Chandra see the Chandra Proposers' Observatory Guide at http://asc.harvard.edu/udocs/docs.

${ }^{2}$ Feigelson et al. (2000) is available at http://www.astro.psu.edu/xray/acis/memos/memoindex.html.
} 
from the gaps between the CCDs. The average aim point, weighted by exposure time, is $\alpha_{2000}=12^{\mathrm{h}}$ $36^{\mathrm{m}} 48^{\mathrm{s}} .1, \delta_{2000}=+62^{\circ} 13^{\prime} 53^{\prime \prime}$. The aim points of the individual observations are separated from the average aim point by $1^{\prime} \cdot 6-3 ! 1$; most are within 2 '. 1 of the average aim point. Due to the large off-axis angle of CCD S2 during these observations, it has low sensitivity. Therefore, we only include data from S2 when these data overlap part of the sky also covered by ACIS-I in other observations.

Background light curves have been inspected for all of the observations. All but one are free from strong flaring due to "space weather" and are stable to within $\approx 20 \%$. The only observation with substantial flaring is 2344 ; during $\approx 30 \mathrm{ks}$ of this observation the background was $\approx 2$ times higher than nominal. However, the data quality during the flaring was still sufficiently high to provide useful scientific information so these data were not excluded; the exclusion of these data would have little impact on the analysis or results presented here.

\subsection{Data Reduction}

The versions of the Chandra X-ray Center (hereafter CXC) pipeline software used for basic processing of the data are listed in Table 1. In the reduction and analysis below, Chandra Interactive Analysis of Observations (CIAO) Version 2 tools were used whenever possible. ${ }^{3}$ Tools for ACIS Realtime Analysis (TARA; Broos et al. 2000) and custom software were also used. ${ }^{4}$

All data were corrected for the radiation damage sustained by the CCDs during the first few months of Chandra operations using the procedure of Townsley et al. (2000). ${ }^{5}$ This procedure partially corrects for the positionally dependent grade distribution due to inefficient charge transfer in the radiation-damaged CCDs. It also partially corrects for quantum efficiency losses, which are most significant in data acquired at $-110^{\circ} \mathrm{C}$ (see Townsley et al. 2000 and Paper II for discussion of the remaining small quantum efficiency losses incurred).

We have removed bad columns, bad pixels, and cosmic ray afterglows as flagged by the CXC using the "status" information in the event files. ${ }^{6}$ We have only used data taken during times within the CXC-generated good-time intervals. The standard pixel randomization was removed as part of the aspect correction procedure described in $\S 3.1 .^{7}$

\footnotetext{
${ }^{3}$ See http://asc.harvard.edu/ciao/.

${ }^{4}$ TARA is available at http://www.astro.psu.edu/xray/docs.

${ }^{5}$ The software associated with the correction method of Townsley et al. (2000) is available at http://www.astro.psu.edu/users/townsley/cti/.

${ }^{6}$ Cosmic ray afterglows occur in front-side illuminated ACIS CCDs when charge from an incident cosmic ray is released slowly, causing a series of spurious events in the same CCD pixel over several sequential frames. These events can resemble a real cosmic X-ray source. See http://cxc.harvard.edu/ciao/advanced_documents.html for information on cosmic ray afterglows.

${ }^{7}$ See http://asc.harvard.edu/cal/Hrma/hrma/misc/oac/dd_psf/dd_randomiz.html.
} 


\section{Data Analysis and Results}

\subsection{Image and Exposure Map Creation}

In this paper we report on the emission detected in four standard X-ray bands: $0.5-8.0 \mathrm{keV}$ (full band), 0.5-2.0 keV (soft band), 2-8 keV (hard band) and 4-8 keV (ultrahard band). We have adopted $8 \mathrm{keV}$ (rather than the often-used $10 \mathrm{keV}$ ) as the full-band, hard-band, and ultrahardband maximum energy because from $8-10 \mathrm{keV}$ the effective area of the HRMA is steeply decreasing with energy while the background is increasing. ${ }^{8}$ Inspection and searching of the data revealed no significant sources in the $8-10 \mathrm{keV}$ band. We have employed the two grade sets defined in Table 2. As described in Paper IV, the use of the "restricted ACIS grade set" in addition to the "standard $A S C A$ grade set" improves our ability to detect faint sources in some cases. All photometry below, however, is reported using the standard $A S C A$ grade set.

We have registered the data sets following $§ 3.1$ of Paper II. Briefly, we registered all the data sets to the coordinate frame of observation 966 using 11-17 bright X-ray sources detected in the individual observations within $\approx 6^{\prime}$ of the aim point; registration is accurate to within $\approx 0^{\prime \prime} 4$. Absolute X-ray source positions were obtained by matching 72 sources from the registered total data set to $1.4 \mathrm{GHz}$ radio sources detected by Richards (2000); these $1.4 \mathrm{GHz}$ sources have accurate ( $\lesssim 0^{\prime \prime} \cdot 3$ ) positions, and the $1.4 \mathrm{GHz}$ coverage encompasses the entire Chandra field. Comparison with these sources allowed us to remove shift, rotation, and plate-scale effects. Chandra positions used in the data set registration and absolute astrometry determination were found using the wavelet-based source detection algorithm WAVDETECT (Dobrzycki et al. 1999; Freeman et al. 2001) following $\S 3.2 .1$.

Figure 2 shows the accuracy of our astrometric solution by matching the full-band Chandra sources presented in $\S 3.2 .1$ with $1.4 \mathrm{GHz}$ sources from Richards (2000). There are $2411.4 \mathrm{GHz}$ sources within the Chandra field. In the matching, we consider only the 74 Chandra sources that match with $1.4 \mathrm{GHz}$ sources to within 2.5 (the 72 sources used to determine the absolute astrometry in the previous paragraph excluded the two outliers in Figure 2 with offsets $>1$ 1'5). The vast majority of these 74 matches are expected to be correct, but $\approx 2$ are expected to be false matches. We also note that in some cases the X-ray source may be offset from the radio source even though both are associated with the same galaxy (e.g., a galaxy with a radio-emitting nuclear starburst that also has an off-nuclear "super-Eddington" X-ray binary). Figure 2 shows that the X-ray positions are usually good to within $\approx 00^{\prime \prime} 6$ for off-axis angles $<5^{\prime}$. At larger off-axis angles, where the HRMA point spread function (PSF) rapidly broadens and becomes complex, the positional accuracy, as expected, degrades. Most sources have positions good to within $1^{\prime \prime}$, but positional offsets up to $\approx 1^{\prime \prime} .5-22^{\prime \prime} 0$ are possible. We do not find any systematic errors in our

\footnotetext{
${ }^{8}$ For further information on the HRMA performance and Chandra background see Chapters 4 and 6 of the Chandra Proposers' Observatory Guide at http://asc.harvard.edu/udocs/docs. Also see the memos on Chandra background at http://asc.harvard.edu/cal/Links/Acis/acis/WWWacis_cal.html.
} 
astrometric solution larger than $\approx 0$ '.25 in size.

In Figures 3 and 4 we show raw and adaptively smoothed images of our field in each of the four standard X-ray bands. These images have been made using the standard ASCA grade set. The adaptively smoothed images have been corrected for spatial variations of the effective exposure time using the exposure maps described below. The exposure maps were adaptively smoothed using the same "scale maps" as for the images themselves, and we excluded regions where the adaptively smoothed effective exposure time was less than $50 \mathrm{ks}$. The adaptively smoothed images were not used for source detection, but they do show many of the detected X-ray sources more clearly than the raw images. Note that some of the sources discussed below are not visible in the adaptively smoothed images; these sources fall below the significance level of the adaptive smoothing used to make the images. In Figure 5 we show a color composite of the adaptively smoothed soft-band, hard-band and ultrahard-band images. Soft sources appear red, moderately hard sources appear green, and the hardest sources appear blue. In Figure 6 we show an adaptively smoothed full-band image of the HDF-N itself and its immediate environs; this image will be discussed further in §3.2.3.

We have made maps of effective exposure time, defined as the equivalent amount of exposure time for a source located at the aim point, following the basic procedure described in $§ 3.2$ of Paper II (see Figure 7). These "exposure maps" take into account the effects of vignetting, gaps between the CCDs, bad column filtering, and bad pixel filtering. One exposure map has been created for each of the standard bands, and the maps were sampled every fourth pixel in both right ascension and declination. In creating these, we have assumed a typical power-law spectrum with a photon index of $\Gamma=1.4$ (this is the slope of the X-ray background in the Chandra band). In Figure 8 we show a cumulative plot of the survey solid angle as a function of effective exposure in the full band.

\subsection{Point-Source Detection and Results}

\subsubsection{Point-Source Detection and Parameterization}

Point-source detection in each standard band was performed with WAVDETECT. WAVDETECT

was run using a " $\sqrt{2}$ sequence" of wavelet scales; scales of $1, \sqrt{2}, 2,2 \sqrt{2}, 4,4 \sqrt{2}$, and 8 pixels were used. We found this choice of wavelet scales to give the best overall performance across the field, but we will also discuss the results for larger wavelet scales in $§ 3.2 .2$. In the WAVDETECT source detection the average aim point defined in $\S 2.2$ was used when calculating off-axis angles. Our key criterion for source detection is that a source must be found with a false-positive probability threshold of $1 \times 10^{-7}$ in at least one of the four standard bands using either the standard $A S C A$ or restricted ACIS grade sets. We have not run WAVDETECT with a low false-positive probability threshold and then performed ex post facto processing of the detected sources to attempt to separate true from spurious detections; such a process would be subjective and counter to the philosophy of WAVDETECT (Freeman et al. 2001; P.E. Freeman 2001, private communication). Our detection criterion is fairly conservative; fainter but real sources are undoubtedly present in the field. However, 
our work on the CDF-N data thus far shows that we have struck an appropriate balance between sensitivity and source reliability.

Conservatively treating the eight images searched as entirely independent, $\approx 5$ false sources are expected statistically for the case of a uniform background. In reality, the background is far from uniform due to the large variation of effective exposure time across the field (see Figure 7). In addition, the background increases near bright point sources due to the PSF "wings." It is difficult to quantify precisely the effects of a non-uniform background upon the performance of WAVDETECT. However, based upon the amount of area where background gradients are present and inspection of the sources detected in this area, we would not expect the number of false sources to be increased by more than a factor of $\sim 2-3$. Less than $\approx 4 \%$ of the sources discussed below should be false.

Extensive analysis of the WAVDETECT source positions revealed that a significant fraction of the sources at off-axis angles $\gtrsim 4^{\prime}$ suffered from centroiding errors of $0.2-2^{\prime \prime}$. These apparently arise as a result of both limitations in the WAVDETECT centroiding method as well as the fact that there is not a single Chandra PSF applicable for any given source (due to the different observation pointings and roll angles described in $\S 2.2$, the spatial profile of any given point source is the superposition of several Chandra PSFs). ${ }^{9}$ Testing and consultation with the CXC showed that the WAVDETECT centroiding could be significantly improved by running WAVDETECT without information about the Chandra PSF (P.E. Freeman and D.E. Harris 2001, private communication). Therefore, in as many cases as possible, we have replaced the original WAVDETECT source positions with those from WAVDETECT runs made excluding PSF information; note that these "no-PSF" runs were only used for positional replacement and not for source detection. Positional replacement was performed for $86 \%$ of the detected sources; for the remaining sources it was not possible due to either a nondetection or a multiple detection in the the no-PSF run. The average positional improvement was $\approx 0^{\prime \prime} .3$ but in some cases was as large as $\approx 1^{\prime \prime} .5$ (as determined by matching with Richards 2000 sources at $1.4 \mathrm{GHz}$ ).

WAVDETECT was also used to search the standard-band images for lower-significance, crossband counterparts to the highly significant sources already detected at the $1 \times 10^{-7}$ level in at least one of the four bands; in these runs we used a false-positive probability threshold of $1 \times 10^{-5}$. We found 110 cross-band counterparts in this manner. Since the spatial-matching requirement greatly reduces the number of pixels being searched, the statistically expected number of false cross-band matches is small $(\lesssim 0.5)$.

All of the standard-band source lists created in the source detection described above were merged to create the point-source catalog given as Table 3. For cross-band matching, a matching radius of $\leq 2.5$ was used for sources within $6^{\prime}$ of the average aim point. For larger off-axis angles, a matching radius of $\leq 44^{\prime \prime} 0$ was used. These matching radii were chosen based on inspection of histograms showing the number of matches obtained as a function of angular separation (see $\S 2$ of

\footnotetext{
${ }^{9}$ Some information on this issue is given as Case \#4460 of the CXC Helpdesk at http://cxc.harvard.edu/helpdesk/.
} 
Boller et al. 1998); with these radii the mismatch probability is $\approx 1 \%$ over the entire field.

Manual correction of the WAVDETECT results was required in several cases. For example, WAVDETECT detections of the brightest components of highly extended sources were removed since these sources will be discussed separately in §3.3. In four cases, we have removed sources whose centroids appear to lie outside the field of view; WAVDETECT had only detected the PSF wings of these sources, and their positions and count rates are not well defined. Manual separation of a few close doubles was required, and we have manually determined the position of each separated source. These sources suffer larger photometric errors due to the difficulty of the separation process. It was also necessary to perform manual photometry for two sources near the edge of the field of view and a few faint sources located close to much brighter sources. We have flagged all sources requiring manual correction in Column 16 of Table 3 (see below).

Below we explain the columns in Table 3.

- Column 1 gives the source number. Sources are listed in order of right ascension.

- Columns 2 and 3 give the right ascension and declination, respectively. These positions have been determined by WAVDETECT when possible. Whenever possible, we quote the position determined in the full band; when a source is not detected in the full band we use, in order of priority, the soft-band position, hard-band position, or ultrahard-band position. In addition, we adopt a soft-band position from a no-PSF run over a full-band position when full-band positional replacement from a no-PSF run was not possible (see above). The priority ordering of position choices above was designed to generally maximize the signal-to-noise of the data being used for positional determination. To avoid truncation error, we quote the positions to higher precision than in the International Astronomical Union approved names beginning with the acronym "CXO HDFN." 10

- Column 4 gives the positional error. Sources within $5^{\prime}$ of the average aim point have positional errors of $0^{\prime \prime} 6$. Sources farther than $5^{\prime}$ from the average aim point have positional errors given by the empirically determined equation:

$$
\Delta=0.6+\left(\frac{\theta-5^{\prime}}{8.75^{\prime}}\right) \operatorname{arcsec} \quad\left(\text { for } \theta>5^{\prime}\right)
$$

where $\Delta$ is the positional error in arcsec and $\theta$ is the off-axis angle in arcmin (compare with Figure 2). The positional error does not appear to be a strong function of the number of source counts (although a mild dependence is probably present); this is largely due to the relatively sharp core of the Chandra PSF. The stated positional errors are for $\approx 90 \%$ confidence, and the accuracy of our astrometric solution is discussed in $\S 3.1$.

\footnotetext{
${ }^{10}$ See http://cxc.harvard.edu/udocs/naming.html.
} 
- Columns 5-8 give the counts in the four standard bands; here and hereafter "FB" indicates full band, "SB" indicates soft band, "HB" indicates hard band, and "UHB" indicates ultrahard band. All values are for the standard $A S C A$ grade set, and they have not been corrected for vignetting. Source counts and $1 \sigma$ statistical errors (from Gehrels 1986) have been calculated using circular aperture photometry; extensive testing showed this method to be more reliable than the WAVDETECT photometry. The circular aperture was centered at the position given in columns 2 and 3 for all bands. A source-free local background has been subtracted, and unexposed regions were masked.

For sources with fewer than 1000 full-band counts, we have chosen the aperture radii based on the encircled-energy function of the Chandra PSF as determined using the CXC's MKPSF software (Feigelson et al. 2000; Jerius et al. 2000; M. Karovska and P. Zhao 2001, private communication). In the soft band where the image quality is the best, the aperture radius was set to the $95 \%$ encircled-energy radius of the PSF, and in the other bands the $90 \%$ encircled-energy radius of the PSF was used. Appropriate aperture corrections were applied to the source counts.

For sources with more than 1000 full-band counts, systematic errors in the aperture corrections often exceed the expected errors from photon statistics when the apertures described in the previous paragraph are used. Therefore, for such sources we used larger apertures to minimize the importance of the aperture corrections; this is appropriate since these bright sources dominate over the background. We set the aperture radii to be twice those used in the previous paragraph and manually inspected these sources to verify that the measurements were not contaminated by neighboring objects.

We have performed several consistency checks to verify the quality of the photometry. For example, we have checked that the sum of the counts measured in the soft and hard bands does not differ from the counts measured in the full band by an amount larger than that expected from measurement error. Systematic errors in our photometry are estimated to be $\lesssim 4 \%$. Photometry more accurate than this will require improved modeling of the Chandra PSF at large off-axis angles by the CXC (M. Karovska and P. Zhao 2001, private communication) as well as detailed treatment of the fact that there is not a single Chandra PSF applicable for any given source (due to the different observation pointings and roll angles described in $\S 2.2$ ). In addition, it will probably be necessary to make the aperture corrections for each source dependent upon its spectral shape.

We have verified that the cosmic ray afterglow removal procedure (see $\S 2.3$ ) has not led to significant systematic photometric errors. Due to the low count rates of our sources, incident $\mathrm{X}$-ray photons are almost never incorrectly flagged as afterglow events.

When a source is not detected in a given band, an upper limit is calculated. All upper limits are determined using circular apertures as above. When the number of counts in the aperture is $\leq 10$, the upper limit is calculated using the Bayesian method of Kraft, Burrows, \& Nousek (1991) for 95\% confidence. The uniform prior used by these authors results in 
fairly conservative upper limits (see Bickel 1992), and other reasonable choices of priors do not materially change our scientific results. For larger numbers of counts in the aperture, upper limits are calculated at the $3 \sigma$ level for Gaussian statistics.

- Column 9 gives the band ratio, defined as the ratio of counts between the hard and soft bands. Errors for this quantity are calculated following the "numerical method" described in $\S 1.7 .3$ of Lyons (1991); this avoids the failure of the standard approximate variance formula when the number of counts is small (see $\S 2.4 .5$ of Eadie et al. 1971). Note that the error distribution is not Gaussian when the number of counts is small. Quoted band ratios have been corrected for differential vignetting between the hard band and soft band using the appropriate exposure maps.

- Column 10 gives the effective photon index $(\Gamma)$ for a power-law model with the Galactic column density. This has been calculated based on the band ratio in column 9 whenever the number of counts is not "low." A source with a low number of counts is defined as being (1) detected in the soft band with $<30$ counts and not detected in the hard band, (2) detected in the hard band with $<15$ counts and not detected in the soft band, (3) detected in both the soft and hard bands, but with $<15$ counts in each, or (4) detected only in the full band. When the number of counts is low, the photon index is poorly constrained and set to $\Gamma=1.4$, a representative value for faint sources that should give reasonable fluxes.

- Column 11 gives the effective exposure time derived from the full-band exposure map (see $\S 3.1$ for details of the exposure maps).

- Columns 12-15 give observed-frame fluxes in the four standard bands; fluxes are in units of $10^{-15} \mathrm{erg}^{-2} \mathrm{~s}^{-1}$. They have been corrected for vignetting but are not corrected for absorption by the Galaxy or intrinsic to the source. For a power-law model with $\Gamma=1.4$, the soft-band and hard-band Galactic absorption corrections are $\approx 4.2 \%$ and $\approx 0.1 \%$, respectively. Fluxes have been computed using the counts in columns $5-8$, the appropriate exposure maps, and the spectral slopes given in column 10 .

- Column 16 gives notes on the sources. "H" and "C" denote objects lying in the HDF-N and the Caltech area, respectively (see Figure 3). "O" refers to objects that have large cross-band positional offsets $(>2$.'5). "NP" refers to objects where the source position could not be updated to that from a no-PSF run. "M" refers to sources where the photometry was performed manually. "S" refers to close double sources where manual separation was required. For further explanation of many of these notes, see the above text in this section on manual correction of the WAVDETECT results.

Some of the sources in Table 3 have been presented in Papers I-IV. The source properties in Table 3 supersede those presented in earlier papers.

Figures 9-12 display the basic properties of the sources in Table 3. In Figure 9 we plot the positions of the sources detected in the soft band and hard band; this format removes the illusory 
effect produced by the changing PSF size across the field of view. Figure 10 shows the distribution of full-band effective exposure time; this plot includes the ten sources that were not detected in the full band (all of the sources that were not detected in the full band were detected in the soft band, and the full-band and soft-band effective exposure times are similar). Figure 11 shows "postage-stamp" images in the full band for all detected sources. Figure 12 displays the band ratio as a function of soft-band count rate for the detected Chandra sources. This plot shows the same qualitative behavior as those from shallower surveys (e.g., Paper IV; Tozzi et al. 2001) and populates the faint flux region more densely. The sources generally become harder at low softband count rates, although there is substantial dispersion. At the lowest soft-band count rates, the source population is heterogeneous; highly absorbed AGN, high-redshift AGN, low-luminosity AGN, starburst galaxies, and normal galaxies all appear to make significant contributions (e.g., Paper II; Paper IV; Paper VI; Tozzi et al. 2001). Understanding in detail the nature and relative contributions of the source populations represented in Figure 12 will require extensive optical spectroscopic and multiwavelength follow-up studies.

We have inspected the sources in Table 3 for spatial extent, and they are generally consistent with being pointlike (see Figure 11). The constraints on spatial extent are 20-40\% worse than for a single ACIS-I observation because, due to the different observation pointings and roll angles described in $\S 2.2$, there is not a single Chandra PSF applicable for any given source. The constraints at off-axis angles of $\lesssim 5^{\prime}$ are also substantially tighter than those at larger off-axis angles. Spatially extended sources are discussed in $\S 3.3$.

In Table 4 we summarize the source detections in the four standard bands, and in Table 5 we summarize the number of sources detected in one band but not another.

Our faintest soft-band sources have $\approx 6$ counts (about one every 1.8 days), and our faintest hard-band sources have $\approx 10$ counts; these sources are detected near the aim point. For a $\Gamma=1.4$ power-law model with the Galactic column density, the corresponding $0.5-2.0 \mathrm{keV}$ and $2-8 \mathrm{keV}$ flux limits are $\approx 2.9 \times 10^{-17} \mathrm{erg} \mathrm{cm}^{-2} \mathrm{~s}^{-1}$ and $\approx 1.9 \times 10^{-16} \mathrm{erg} \mathrm{cm}^{-2} \mathrm{~s}^{-1}$, respectively. Of course, these flux limits vary across the field of view. Using the restricted ACIS grade set, the background level in our region of highest exposure (see Figures 7 and 8 ) is $3.6 \times 10^{-2}$ count pixel $^{-1}$ in the soft band and $1.1 \times 10^{-1}$ count pixel $^{-1}$ in the hard band. In both of these bands we are far from being background limited for point source detection near the aim point.

\subsubsection{Supplementary Point Sources at Large Off-Axis Angles}

Unfortunately, there is no single choice of wavelet scales in WAVDETECT that provides perfect performance across an entire Chandra field (Freeman et al. 2001; P.E. Freeman 2001, private communication). Compromises are required due to, for example, the dependence of the PSF size and shape on off-axis angle. In addition, due to the different observation pointings and roll angles described in $\S 2.2$, there is not a single Chandra PSF applicable for any given source in this particular 
field. The wavelet scales used in $§ 3.2 .1$ have been empirically found to provide very good performance across most of the field, but adding further large wavelet scales (e.g., $8 \sqrt{2}$ and 16 pixels) can improve the detection effectiveness at large off-axis angles where the PSF is broad. However, the addition of these scales can cause real sources at smaller off-axis angles to be missed due to the incorrect merging of two sources. In addition, the "rejection rule" of WAVDETECT, designed to suppress Poisson fluctuations on scales smaller than the PSF, has limitations that can cause incorrect source rejections when large wavelet scales are employed (see $§ 3.2 .3$ of Freeman et al. 2001; P.E. Freeman 2001, private communication). For example, incorrect source rejections may occur when only the relatively sharp core of the PSF is apparent above the background level.

To address this issue and provide the best possible performance at large off-axis angles, we have created a supplementary catalog of 11 additional sources found by WAVDETECT at large off-axis angles when wavelet scales of $8 \sqrt{2}$ and 16 pixels are added to those used in $\S 3.2 .1$ (see Table 6 ). We have followed the same basic methodology as was used when making the main catalog (see §3.2.1); we have again adopted a false-positive probability threshold of $1 \times 10^{-7}$ as the key criterion for source detection. The catalog columns are the same as for the main catalog. Because the resulting sources tend to be the weakest ones detectable at the largest off-axis angles, their properties are in general less well defined than those of the sources in the main catalog. We have manually inspected these sources to confirm their reality.

\subsubsection{Comparison with Previous Results for the HDF-N}

A comparison of the Chandra sources detected in the HDF-N here and in Paper IV gives good agreement. All but one of the 12 sources detected in Paper IV are detected here as well. The one source not detected here is CXOHDFN J123643.9+621249; this faint source is known to be variable (see Paper IV) and has dropped in flux, leading to a reduced signal-to-noise ratio in the longer observation. ${ }^{11}$ There is still a notable positive fluctuation at the location of CXOHDFN J123643.9+621249 (see Figure 6), and it is detected in the soft band if WAVDETECT is run with a false-positive probability threshold of $1 \times 10^{-6}$. One other small difference from Paper IV is that the position of CXOHDFN J123649.4+621347 has moved closer to the nucleus of its $z=0.089$ elliptical host galaxy (see $§ 3.1$ of Paper IV); it is no longer clear that this faint X-ray source lies outside the nucleus.

With the additional data presented here, two new HDF-N X-ray sources have been discovered: CXOHDFN J123656.6+621245 and CXOHDFN J123657.4+621210. Both sources appear to be associated with optically bright HDF-N galaxies, extending the trend noted in Paper IV. The first is W96 source 3-610.1 at $z=0.518$ (Cohen et al. 2000), one of the optically brightest $\left(V_{606}=21.30\right.$ )

\footnotetext{
${ }^{11}$ We note that there may be other faint, variable sources like CXOHDFN J123643.9+621249 that could be recovered by searching the data using many time segments. However, addressing this issue comprehensively is a challenging task and is beyond the scope of this paper.
} 
spirals in the HDF-N. The second is W96 source 3-965.0 at $z=0.665$ (Cohen et al. 2000), a bright $\left(V_{606}=22.23\right)$ elliptical near the edge of the HDF-N. The full-band X-ray luminosities of these galaxies of $\approx 1 \times 10^{41} \mathrm{erg} \mathrm{s}^{-1}$ and $\approx 3 \times 10^{41} \mathrm{erg} \mathrm{s}^{-1}$, respectively, can be explained via either low-luminosity AGN or stellar remnants (e.g., X-ray binaries). Both of these galaxies were included in the stacking analysis of Paper IV, and their individual detections with additional data support the validity of the stacking analysis.

We note that there are several other positive fluctuations in the HDF-N visible in Figure 6. While some of these align with bright HDF-N galaxies (e.g., the positive fluctuation near $\alpha_{2000}=12^{\mathrm{h}}$ $36^{\mathrm{m}} 52^{\mathrm{s}} 8, \delta_{2000}=+62^{\circ} 13^{\prime} 54^{\prime \prime}$ aligns with W96 source $2-736.0$, a $z=1.355$ irregular) and may well be real, they are not formally detected according to the criteria in $\S 3.2 .1$ and will not be discussed further here.

\subsubsection{Number Counts for the Point Sources}

We have calculated cumulative number counts, $N(>S)$, for the soft and hard bands using the data in Table 3 and the basic method described in Gioia et al. (1990). The results are shown in Figure 13, along with some results from ROSAT and ASCA. The CDF-N number counts do not extend to higher fluxes due to the limited number of bright sources available in our data.

We have been conservative in our source selection, taking several steps to prevent incompleteness (due to the varying sensitivity as a function of spatial position) from affecting the $N(>S)$ curves. To avoid incompleteness in the soft band, we have only used data which satisfy the softband requirements in Table 7 on (1) distance from the average aim point and (2) soft-band effective exposure time. The second requirement has the effect of removing areas affected by the gaps between the CCDs, where incompleteness is difficult to assess (see Figure 7). In Table 7 we also list the solid angle satisfying the above requirements. We have only extended the soft-band $N(>S)$ down to $4.2 \times 10^{-17} \mathrm{erg} \mathrm{cm}^{-2} \mathrm{~s}^{-1}$ since, even near the average aim point, incompleteness may affect the $N(>S)$ at fainter fluxes; sources below this flux typically have $\lesssim 8$ soft-band counts. At this flux limit, the source density is $7100_{-940}^{+1100} \mathrm{deg}^{-2}$. The Chandra and ROSAT constraints on the soft-band $N(>S)$ are consistent in the limited region of overlap. We have fit the CDF-N $N(>S)$ curve in the flux range $5 \times 10^{-17} \mathrm{erg} \mathrm{cm}^{-2} \mathrm{~s}^{-1}$ to $2 \times 10^{-15} \mathrm{erg} \mathrm{cm}^{-2} \mathrm{~s}^{-1}$ using a maximum likelihood technique (Murdoch, Crawford, \& Jauncey 1973). The best fit is

$$
N(>S)=3970\left(\frac{S}{1 \times 10^{-16}}\right)^{-0.67 \pm 0.14} .
$$

To avoid incompleteness in the hard band, we have only used data which satisfy the hardband requirements in Table 7 . We have only extended the hard-band $N(>S)$ down to $3.8 \times$ $10^{-16} \mathrm{erg}^{-2} \mathrm{~s}^{-1}$ since, even near the average aim point, incompleteness may affect the $N(>S)$ at fainter fluxes; sources below this flux typically have $\lesssim 12$ hard-band counts. At this flux limit, 
the source density is $4200_{-580}^{+670} \mathrm{deg}^{-2}$. There is very little overlap between the Chandra constraints on the hard-band $N(>S)$ and those from earlier missions. The best fit to the CDF-N $N(>S)$ curve in the flux range above $1.5 \times 10^{-15} \mathrm{erg}^{-2} \mathrm{~s}^{-1}$ is

$$
N(>S)=2820\left(\frac{S}{1 \times 10^{-15}}\right)^{-1.0 \pm 0.3}
$$

At fainter fluxes the number counts clearly flatten (see Figure 13b), and parameterization of them requires a detailed analysis of the differential counts (see Jauncey 1975 and references therein). This analysis, along with a more detailed study of the number counts in the CDF-N, will be presented in G.P. Garmire et al., in preparation.

We have also experimented with somewhat less conservative source-selection methods than those used above, and these give $N(>S)$ curves that are consistent with those presented above to within the expected statistical uncertainties. In the hard band, in fact, varying the source selection method gives almost identical results.

In Figure 14 we compare our $N(>S)$ curves with those from Mushotzky et al. (2000) and Tozzi et al. (2001). In the soft band, our $N(>S$ ) curve is above those of both Mushotzky et al. (2000) and Tozzi et al. (2001), even at quite bright X-ray fluxes. However, the results are not discrepant given the error bars, and any small differences could be due to field-to-field "cosmic variance." In the hard band, our $N(>S)$ curve is consistent with those of Mushotzky et al. (2000) and Tozzi et al. (2001) at fluxes above $3 \times 10^{-15} \mathrm{erg} \mathrm{cm}^{-2} \mathrm{~s}^{-1}$, and at fainter fluxes it is intermediate between the two curves.

\subsection{Extended-Source Detection and Results}

We have searched the standard-band images for extended X-ray sources using the CXC's Voronoi Tessellation and Percolation algorithm VTPDEtect (Ebeling \& Wiedenmann 1993; Dobrzycki et al. 1999). We have used a false-positive probability threshold of $1 \times 10^{-7}$, and we require at least 50 counts per source. Extended source detections were checked by inspection of adaptively smoothed images.

In Figure 15 we show the two most significant extended sources revealed by our source detection: CXOHDFN J123620.0+621554 and CXOHDFN J123756.0+621506. Both of these sources are detected most clearly in the soft-band image. We have checked for possible problems with these source detections and find none. The exposure map is relatively smooth near both of these sources, so background gradients should not have confused the extended source detection. In addition, both of these sources are visible in adaptively smoothed soft-band images made using only data with roll angles of $36.4-44.5^{\circ}$ or $134.3-143.8^{\circ}$ (see Table 1 ); this argues against an instrumental origin of these sources. CXOHDFN J123620.0+621554 has $274 \pm 37$ soft-band counts and no obvious 
optical counterpart in the I-band image of Barger et al. (1999), suggesting a fairly high redshift group or cluster. The counts for extended sources here and hereafter were determined with manual aperture photometry excluding point sources and regions of strongly varying background; for CXOHDFN J123620.0+621554 we used an elliptical aperture with semimajor axis $45^{\prime \prime}$, semiminor axis $25^{\prime \prime}$, and position angle $145^{\circ}$. CXOHDFN J123756.0+621506 has $303 \pm 26$ soft-band counts (in an elliptical aperture with semimajor axis $25^{\prime \prime}$, semiminor axis $18^{\prime \prime}$, and position angle $\left.55^{\circ}\right)$. In the $R$-band image of Liu et al. (1999), it coincides with a pair of optically bright galaxies that appear to be interacting; the X-ray source is likely to be a low-to-moderate redshift group.

One additional extended source detected by VTPDETECT with lower significance than the two above is CXOHDFN J123645.0+621142 (see Figure 16); we consider this source to be marginally detected. This source is notable because it is in the HDF-N itself near the $z=1.013$ Fanaroff-Riley I (FR I) radio galaxy VLA J123644.3+621133 and a number of other $z \approx 1.01$ objects (e.g., Richards et al. 1998). In Paper IV we detected the FR I (as CXOHDFN J123644.3+621132) and searched for cluster X-ray emission in its vicinity because FR I sources are often located in clusters of galaxies. No cluster emission was found, but with the additional data here it appears likely that such emission was present just below the detection threshold attainable in Paper IV. The FR I lies within the emission but appears offset from its (poorly defined) center. CXOHDFN J123645.0+621142 has $\approx 100$ soft-band counts (in a circular aperture with radius $30^{\prime \prime}$ ). Because there are substantially more counts in the putative cluster emission than from the FR I, we are confident that this emission is not merely due to counts from the FR I in the wings of the PSF.

Inspection of the raw and adaptively smoothed soft-band images revealed the likely presence of diffuse emission near the positions 123557.0+621551, 123704.6+621652, and 123721.2+621526. Possible groups or clusters are apparent in the $R$-band image of Liu et al. (1999) or the $I$-band image of Barger et al. (1999) near these positions. Optical spectroscopy is required for confirmation.

Dawson et al. (2001) have discovered a $z=0.85$ cluster centered near $\alpha_{2000}=12^{\mathrm{h}} 36^{\mathrm{m}} 39.6$, $\delta_{2000}=+62^{\circ} 15^{\prime} 54^{\prime \prime}$. Unfortunately, this position is near one of the strongest CCD gap features in the exposure map (see Figure 7). Inspection of the adaptively smoothed soft-band image for diffuse emission shows a possible $\approx 50$ event enhancement near cluster member F 36397+1547 (see Table 4 of Dawson et al. 2001), but this is not formally detected by VTPDETECT and could be due to a faint point source. We do clearly detect X-ray emission from cluster member F $36421+1545$; this source appears to be pointlike.

We have also inspected the Chandra images near the Wide Angle Tail (WAT) radio galaxy VLA J123725.7+621128 (Muxlow et al. 1999) since WATs are often found in clusters of galaxies. We find no hint of any extended X-ray emission centered near this source.

Extended sources will be discussed in further detail in F.E. Bauer et al., in preparation. 


\section{Conclusions and Summary}

These CDF-N observations will contribute greatly to the resolution of some of the outstanding questions in extragalactic X-ray astronomy. Near the aim point, they have reached soft-band and hard-band source densities of $7100_{-940}^{+1100} \mathrm{deg}^{-2}\left(\right.$ at $4.2 \times 10^{-17} \mathrm{erg} \mathrm{cm}^{-2} \mathrm{~s}^{-1}$ ) and $4200_{-580}^{+670} \mathrm{deg}^{-2}$ (at $3.8 \times 10^{-16} \mathrm{erg} \mathrm{cm}^{-2} \mathrm{~s}^{-1}$ ), respectively. This paper has described the details of the observations, data reduction, and technical analysis and presented $1 \mathrm{Ms}$ source catalogs. It will require many years of work to investigate all of the presented sources in detail. This survey should remain one of the deepest ever made from $0.5-8.0 \mathrm{keV}$ until missions such as the X-ray Evolving Universe Spectroscopy (XEUS) mission begin operation, ${ }^{12}$ and we hope to extend this survey to an exposure of 5-10 Ms given the continued operation of Chandra; we have been allocated an additional $1 \mathrm{Ms}$ in Chandra Cycle 3 (all data will become public immediately). The current $1 \mathrm{Ms}$ survey is far from the limit of Chandra's capability. The detector background is so low that, with appropriate grade screening, we will not fully enter the background-limited regime near the aim point for exposure times of $\lesssim 5 \mathrm{Ms}$ (for the full band; the situation is even better for the soft band due to its substantially lower background). A 5 Ms exposure would achieve soft-band and hard-band sensitivities of $\approx 6 \times 10^{-18} \mathrm{erg} \mathrm{cm}^{-2} \mathrm{~s}^{-1}$ and $\approx 4 \times 10^{-17} \mathrm{erg} \mathrm{cm}^{-2} \mathrm{~s}^{-1}$, respectively, as well as provide detailed spectral, variability, and morphological constraints on the sources in the present catalog. It would provide key information on the existence and nature of the sources to be targeted by future missions such as $X E U S$, laying the groundwork for these missions. XMM-Newton will perform deeper surveys in the $\approx 8-12 \mathrm{keV}$ band, but cross-correlation of the sources found by $X M M$ Newton with those presented here will still be important to refine the XMM-Newton positions and minimize source confusion.

The source catalogs and images shown in Figure 3 are available on the World Wide Web. ${ }^{13}$ We will continue to improve the source catalogs as better calibration information, analysis methods and software become available. For example, we plan to optimize the searching for variable sources and search more sensitively for X-ray sources that correlate with sources at other wavelengths. Improved searching for diffuse sources will be performed as the Chandra background becomes better understood.

This work would not have been possible without the enormous efforts of the entire Chandra and ACIS teams. We thank P.E. Freeman, J. Gaffney, D.E. Harris, M. Karovska, C. Liu, and P. Zhao for helpful discussions and sharing data. We gratefully acknowledge the financial support of NASA grant NAS 8-38252 (GPG, PI), NSF CAREER award AST-9983783 (WNB, DMA, FEB), NASA GSRP grant NGT 5-50247 and the Pennsylvania Space Grant Consortium (AEH), NSF grant AST-9900703 (DPS), NASA Hubble Fellowship grant HF-01117.01-A and NSF grant AST-0084847

\footnotetext{
${ }^{12}$ For details on XEUS see http://astro.estec.esa.nl/SA-general/Projects/XEUS/.

${ }^{13}$ See http://www.astro.psu.edu/users/niel/hdf/hdf-chandra.html.
} 
(AJB), and NSF grant AST-0084816 (LLC).

\section{REFERENCES}

Alexander, D.M., et al. 2001, AJ, in press (Paper VI; astro-ph/0107450)

Barger, A.J., Cowie, L.L., Trentham, N., Fulton, E., Hu, E., Songaila, A., \& Hall, D., 1999, AJ, 117,102

Barger, A.J., Cowie, L.L., Bautz, M.W., Brandt, W.N., Garmire, G.P., Hornschemeier, A.E., Ivison, R.J., Owen, F.N. 2001, AJ, submitted

Bickel, P.J. 1992, in Statistical Challenges in Modern Astronomy, eds. Feigelson, E.D., \& Babu, G.J. (Springer-Verlag, New York), p. 320

Boller, Th., Bertoldi, F., Dennefeld, M., \& Voges, W. 1998, A\&AS, 129, 87

Brandt, W.N., et al. 2001a, AJ, 122, 1 (Paper IV)

Brandt, W.N., Hornschemeier, A.E., Schneider, D.P., Alexander, D.M., Bauer, F.E., Garmire, G.P., \& Vignali, C. 2001b, ApJ, 558, L5 (Paper VII)

Broos, P., et al. 2000, User's Guide for the TARA Package. The Pennsylvania State University, University Park

Cohen, J.G., Hogg, D.W., Blandford, R., Cowie, L.L., Hu, E., Songaila, A., Shopbell, P., \& Richberg, K. 2000, ApJ, 538, 29

Dawson, S., Stern, D., Bunker, A.J., Spinrad, H., \& Dey, A. 2001, AJ, in press (astro-ph/0105043)

Dobrzycki, A., Ebeling, H., Glotfelty, K., Freeman, P., Damiani, F., Elvis, M., \& Calderwood, T. 1999, Chandra Detect 1.0 User Guide. Chandra X-ray Center, Cambridge

Eadie, W.T., Dryard, D., James, F.E., Roos, M., \& Sadoulet, B. 1971, Statistical Methods in Experimental Physics. North-Holland, Amsterdam

Ebeling, H. \& Wiedenmann, G. 1993, Phys. Rev. E, 47, 704

Ebeling, H., White, D.A., \& Rangarajan, F.V.N. 2001, MNRAS, submitted

Feigelson, E.D., Broos, P.S., \& Gaffney, J. 2000, Memo on the Optimal Extraction Radius for ACIS Point Sources. The Pennsylvania State University, University Park

Ferguson, H.C, Dickinson, M., \& Williams, R., 2000, ARA\&A, 38, 667

Forman, W., Jones, C., Cominsky, L., Julien, P., Murray, S., Peters, G., Tananbaum, H., \& Giacconi, R. 1978, ApJS, 38, 357

Freeman, P.E., Kashyap, V., Rosner, R., \& Lamb, D.Q. 2001, ApJ, submitted

Garmire, G.P., et al. 2001, ApJ, submitted (Paper III)

Gehrels, N. 1986, ApJ, 303, 336 
Gendreau, K.C., Barcons, X., \& Fabian, A.C. 1998, MNRAS, 297, 41

Gioia, I., Maccacaro, T., Schild, R., Wolter, A., Stocke, J., Morris, S., \& Henry, J.P. 1990, ApJS, 72,567

Hasinger, G., Burg, R., Giacconi, R., Hartner, G., Schmidt, M., Trümper, J., \& Zamorani, G. 1993, A\&A, 275, 1

Hasinger, G., et al. 1998, A\&A, 340, L27

Hasinger, G., et al. 2001, A\&A, 365, L45

Hogg, D.W., et al. 2000, ApJS, 127, 1

Hornschemeier, A.E., et al. 2000, ApJ, 541, 49 (Paper I)

Hornschemeier, A.E., et al. 2001, ApJ, 554, 742 (Paper II)

Jauncey, D.L. 1975, ARAA, 13, 23

Jerius, D., Donnelly, R.H., Tibbetts, M.S., Edgar, R.J., Gaetz, T.J., Schwartz, D.A., Van Speybroeck, L.P., \& Zhao, P. 2000, Proc. SPIE, 4012, 17

Kraft, R.P., Burrows, D.N. \& Nousek, J.A. 1991, ApJ, 374, 344

Lehmann, I., et al. 2001, A\&A, 371, 833

Liu, C.T., Petry, C.E., Impey, C.D., \& Foltz, C.B. 1999, AJ, 118, 1912

Livio, M., Fall, S.M., \& Madau, P. 1998, The Hubble Deep Field. Cambridge University Press, Cambridge

Lyons, L. 1991, Data Analysis for Physical Science Students. Cambridge University Press, Cambridge

Mason, K.O., et al. 2000, MNRAS, 311, 456

Murdoch, H.S, Crawford, D.F., \& Jauncey, D.L. 1973, ApJ, 183, 1

Mushotzky, R.F., Cowie, L.L., Barger, A.J., \& Arnaud, K.A. 2000, Nature, 404, 459

Muxlow, T.W.B., Wilkinson, P.N., Richards, A.M.S., Kellermann, K.I., Richards, E.A., \& Garrett, M.A. 1999, New Astronomy Reviews, 43, 623

Ogasaka, Y., et al. 1998, Astr Nach, 319, 43

Richards, E.A. 2000, ApJ, 533, 611

Stark, A.A., Gammie, C.F., Wilson, R.W., Bally, J., Linke, R.A., Heiles, C., \& Hurwitz, M. 1992, ApJS, 79, 77

Townsley, L.K., Broos, P.S., Garmire, G.P., \& Nousek, J.A. 2000, ApJ, 534, L139

Tozzi, P., et al. 2001, ApJ, in press (astro-ph/0103014)

Ueda, Y., et al. 1998, Nature, 391, 866

Voges, W., et al. 1999, A\&A, 349, 389 
Watson, M.G., et al. 2001, A\&A, 365, L51

Weisskopf, M.C., Tananbaum, H.D., Van Speybroeck, L.P., \& O’Dell, S.L. 2000, Proc. SPIE, 4012, 2

Wilkes, B.J., et al. 2001, in Proceedings of the New Era of Wide Field Astronomy, eds. Clowes, R.G., Adamson, A.J., \& Bromage, G.E. (ASP Press, San Francisco), in press (astro-ph/0011377)

Williams, R.E., et al. 1996, AJ, 112, 1335 (W96) 
Table 1. Journal of Chandra Observations

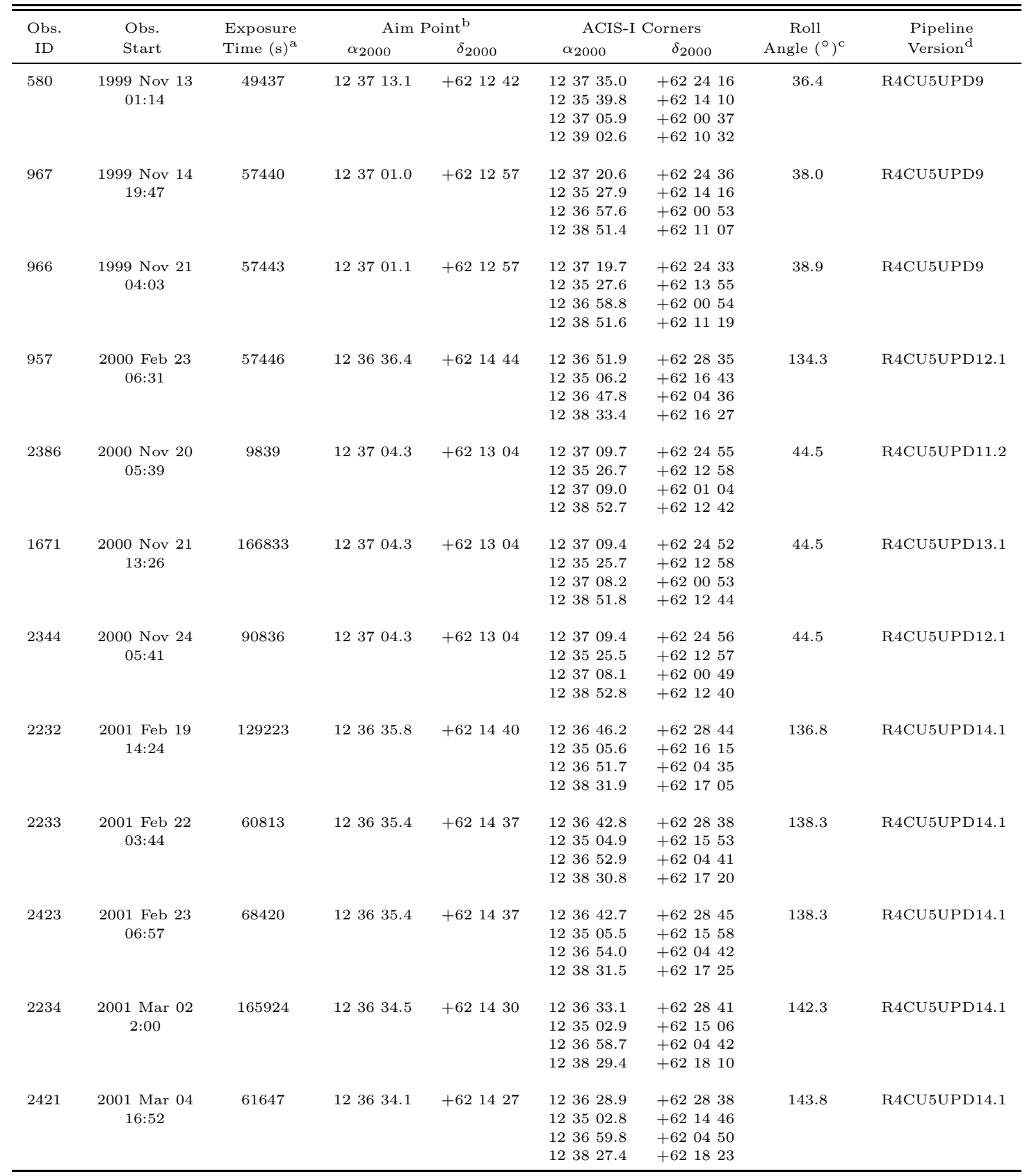

Note. - The focal-plane temperature was $-110^{\circ} \mathrm{C}$ during the first three observations and $-120^{\circ} \mathrm{C}$ during the others.

${ }^{a}$ All observations were continuous. These times have been corrected for lost exposure due to the $0.041 \mathrm{~s}$ read-out time per CCD frame, and we have also removed short time intervals with bad satellite aspect.

${ }^{\mathrm{b}}$ The average aim point, weighted by exposure time, is $\alpha_{2000}=12^{\mathrm{h}} 36^{\mathrm{m}} 48^{\mathrm{s}} .1, \delta_{2000}=+62^{\circ} 13^{\prime} 53^{\prime \prime}$.

${ }^{c}$ Roll angle describes the orientation of the Chandra instruments on the sky. The angle is between $0-360^{\circ}$, and it increases to the West of North (opposite to the sense of traditional position angle).

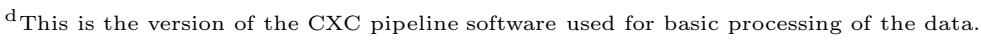


Table 2. Grade Sets

\begin{tabular}{lll}
\hline \hline \multicolumn{1}{c}{ Name } & \multicolumn{1}{c}{ Band } & \multicolumn{1}{c}{ Grades } \\
\hline $\begin{array}{l}\text { Standard } A S C A \\
\text { grade set }\end{array}$ & Full $(0.5-8.0 \mathrm{keV})$ & ASCA grades $0,2,3,4,6$ \\
& Soft $(0.5-2.0 \mathrm{keV})$ & $A S C A$ grades $0,2,3,4,6$ \\
& Hard $(2-8 \mathrm{keV})$ & $A S C A$ grades $0,2,3,4,6$ \\
& Ultrahard $(4-8 \mathrm{keV})$ & $A S C A$ grades $0,2,3,4,6$ \\
Restricted ACIS & Full $(0.5-8.0 \mathrm{keV})$ & ACIS grades 0, 2, 8, 16, 64 \\
grade set & Soft $(0.5-2.0 \mathrm{keV})$ & ACIS grades 0, 64 \\
& Hard $(2-8 \mathrm{keV})$ & ACIS grades 0, 2, 8, 16 \\
& Ultrahard $(4-8 \mathrm{keV})$ & ACIS grades 0, 2, 8, 16 \\
\hline
\end{tabular}

Table 3. For this table please see the World Wide Web site listed in Footnote 13. = 
Table 4. Summary of Chandra Source Detections

\begin{tabular}{lccccc}
\hline \hline $\begin{array}{c}\text { Energy } \\
\text { Band }\end{array}$ & $\begin{array}{c}\text { Number of } \\
\text { Sources }^{\mathrm{a}}\end{array}$ & \multicolumn{4}{c}{ Detected Counts Per Source } \\
\hline Full & 360 & 9100.7 & 7.8 & 75.8 & 289.0 \\
Soft & 325 & 6633.0 & 5.5 & 44.6 & 211.5 \\
Hard & 265 & 2481.0 & 6.0 & 50.7 & 130.2 \\
Ultrahard & 145 & 792.0 & 6.4 & 36.0 & 69.2 \\
\hline
\end{tabular}

${ }^{a}$ There are 370 independent X-ray sources detected in total with a falsepositive probability threshold of $1 \times 10^{-7}$. We have included cross-band counterparts from the WAVDETECT runs with a false-positive probability threshold of $1 \times 10^{-5}$ (see $\S 3.2 .1$ ).

Table 5. Sources Detected in One Band but Not Another

\begin{tabular}{lcccc}
\hline \hline \multirow{2}{*}{ Detection } & \multicolumn{4}{c}{ Non-Detection Energy Band } \\
Energy Band & Full & Soft & Hard & Ultrahard \\
\hline \multirow{2}{*}{ Full } & 0 & 45 & 95 & 215 \\
Soft & 10 & 0 & 98 & 202 \\
Hard & 0 & 38 & 0 & 121 \\
Ultrahard & 0 & 22 & 1 & 0 \\
\hline
\end{tabular}

Note. - For example, there were 45 sources detected in the full band but not in the soft band.

Table 6. For this table please see the World Wide Web site listed in Footnote 13. 
Table 7. Source Selection for the Number Counts

\begin{tabular}{cccc}
\hline \hline $\begin{array}{c}\text { Flux Range } \\
\left(\mathrm{erg} \mathrm{cm} \mathrm{cm}^{-2}\right)\end{array}$ & $\begin{array}{c}\text { Maximum Source } \\
\text { Selection Radius }^{\mathrm{a}}\end{array}$ & $\begin{array}{c}\text { Minimum Effective } \\
\text { Exposure Time }(\mathrm{ks})\end{array}$ & $\begin{array}{c}\text { Selected Solid } \\
\text { Angle }\left(\operatorname{arcmin}^{2}\right)^{\mathrm{b}}\end{array}$ \\
\hline \multicolumn{4}{c}{ Soft Band } \\
\hline$(4.2-20) \times 10^{-17}$ & $3^{\prime}$ & 870 & 18.75 \\
$>2 \times 10^{-16}$ & $5^{\prime}$ & 870 & 50.26 \\
\hline \multicolumn{5}{c}{ Hard Band } \\
\hline$(3.8-8) \times 10^{-16}$ & $3^{\prime}$ & 870 & 17.89 \\
$>4.5 \times 10^{-15}$ & $5^{\prime}$ & 870 & 45.58 \\
\hline
\end{tabular}

${ }^{a}$ Only sources with angular separations from the average aim point smaller than this value were used for the number counts calculations in the corresponding flux range.

${ }^{\mathrm{b}}$ Amount of solid angle satisfying the spatial and effective exposure time filtering criteria. 


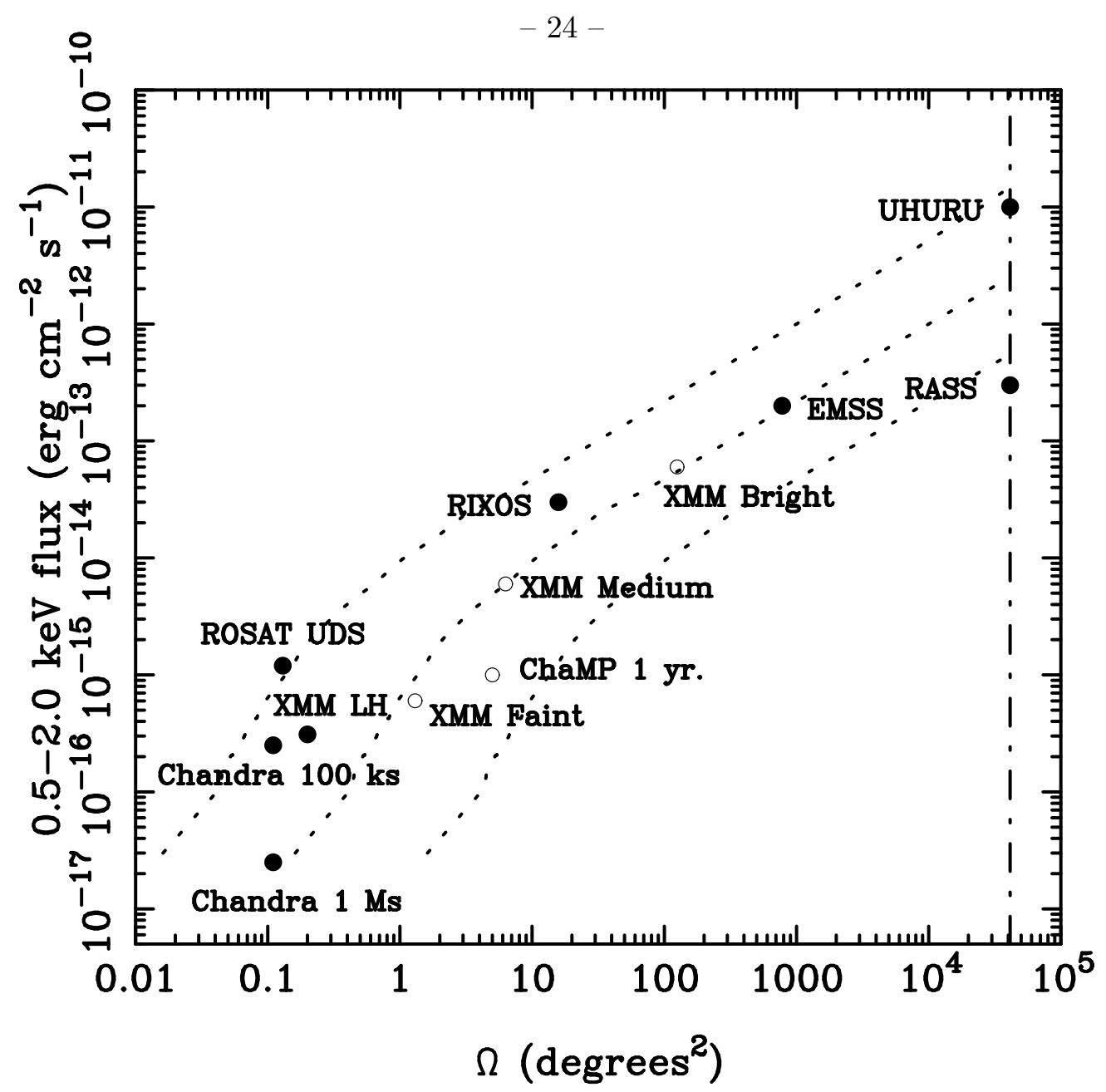

Fig. 1.- Distribution of some extragalactic X-ray surveys in the $0.5-2.0 \mathrm{keV}$ flux limit versus solid angle, $\Omega$, plane. Shown are the Uhuru survey (e.g., Forman et al. 1978), the ROSAT All-Sky Survey (RASS; e.g., Voges et al. 1999), the Einstein Extended Medium-Sensitivity Survey (EMSS; e.g., Gioia et al. 1990), the ROSAT International X-ray/Optical Survey (RIXOS; e.g., Mason et al. 2000), the XMM-Newton Serendipitious Surveys (XMM-Newton Bright, XMM-Newton Medium, $X M M-N e w t o n$ Faint; e.g., Watson et al. 2001), the Chandra Multiwavelength Project (ChaMP; e.g., Wilkes et al. 2001), the ROSAT Ultra Deep Survey (UDS; e.g., Lehmann et al. 2001), the deep XMM-Newton survey of the Lockman Hole (XMM-Newton LH; e.g., Hasinger et al. 2001), Chandra 100 ks surveys (e.g., Mushotzky et al. 2000), and Chandra 1 Ms surveys (i.e., the CDF-N survey and the Chandra Deep Field South survey). Solid dots are for surveys that have been completed, and open circles are for surveys that are in progress. Clearly, each of the surveys shown has a range of flux limits across its solid angle (due to effects such as off-axis PSF broadening and vignetting); we have generally shown the most sensitive flux limit. The dotted curves show, from top to bottom, the loci of 100, 1000, and 10000 0.5-2.0 keV sources (these have been calculated using the number counts of Hasinger et al. 1998, Paper III, and §3.2.4); for example, a 1 degree $^{2}$ survey with a $0.5-2.0 \mathrm{keV}$ flux limit of $9.5 \times 10^{-15} \mathrm{erg} \mathrm{cm}^{-2} \mathrm{~s}^{-1}$ will detect $\approx 100$ sources. The vertical dot-dashed line shows the solid angle of the whole sky. 


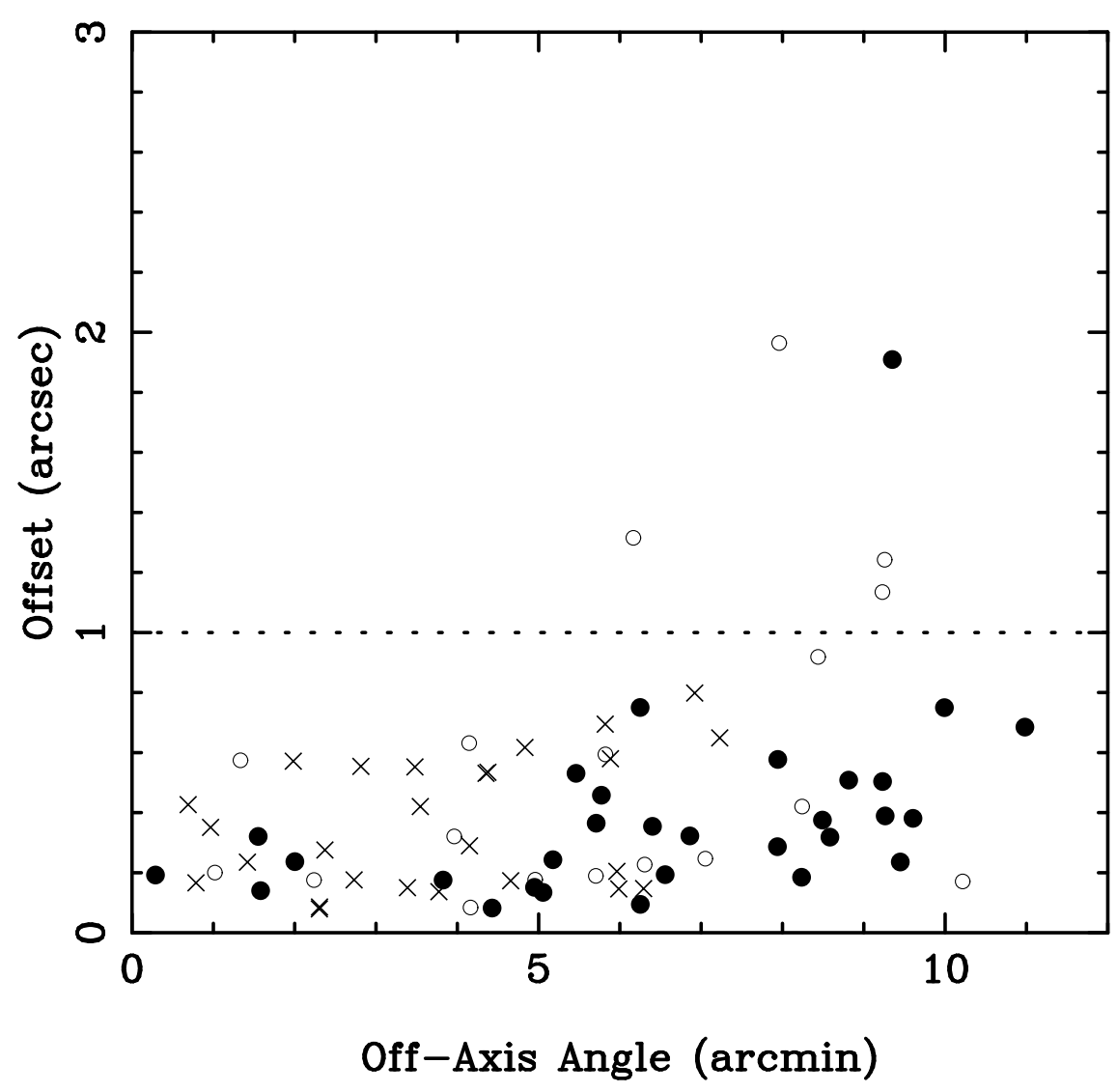

Fig. 2.- Positional offset versus off-axis angle for full-band Chandra sources that match with $1.4 \mathrm{GHz}$ sources (from Richards 2000) to within 2".5. The crosses are Chandra sources with 10-50 counts, the open circles are Chandra sources with 50-100 counts, and the solid dots are Chandra sources with $>100$ counts. The X-ray positions are usually good to within $\approx 0$ "' 6 for off-axis angles $<5^{\prime}$. At larger off-axis angles, where the HRMA PSF rapidly broadens and becomes complex, the positional accuracy, as expected, degrades. The Chandra source positions have been determined following $§ 3.2 .1$; positions from no-PSF runs were used whenever possible. 


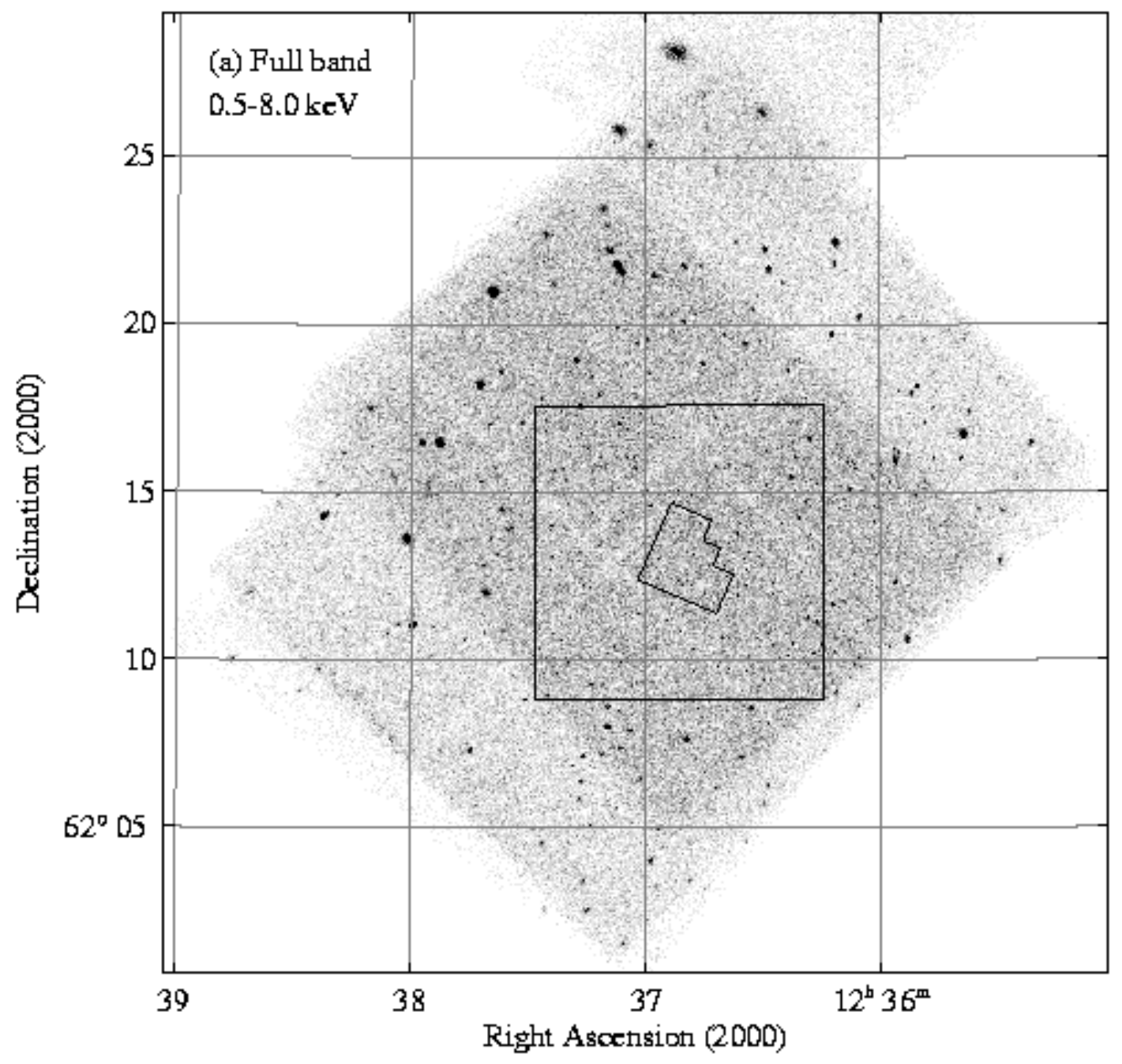

Fig. 3.- Images of the CDF-N in the (a) full band, (b) soft band, (c) hard band, and (d) ultrahard band. These images have been made using the standard $A S C A$ grade set (see Table 2), and they are binned by a factor of four in both right ascension and declination. The light grooves running through the images correspond to the gaps between the CCDs. The small polygon indicates the HDF-N, and the large square indicates the area covered by the Caltech Faint Field Galaxy Redshift Survey (e.g., Hogg et al. 2000; hereafter the "Caltech area"). Note: Only one of the four pages of images could be included here; please see the World Wide Web site listed in Footnote 13 for the version with all the images. 


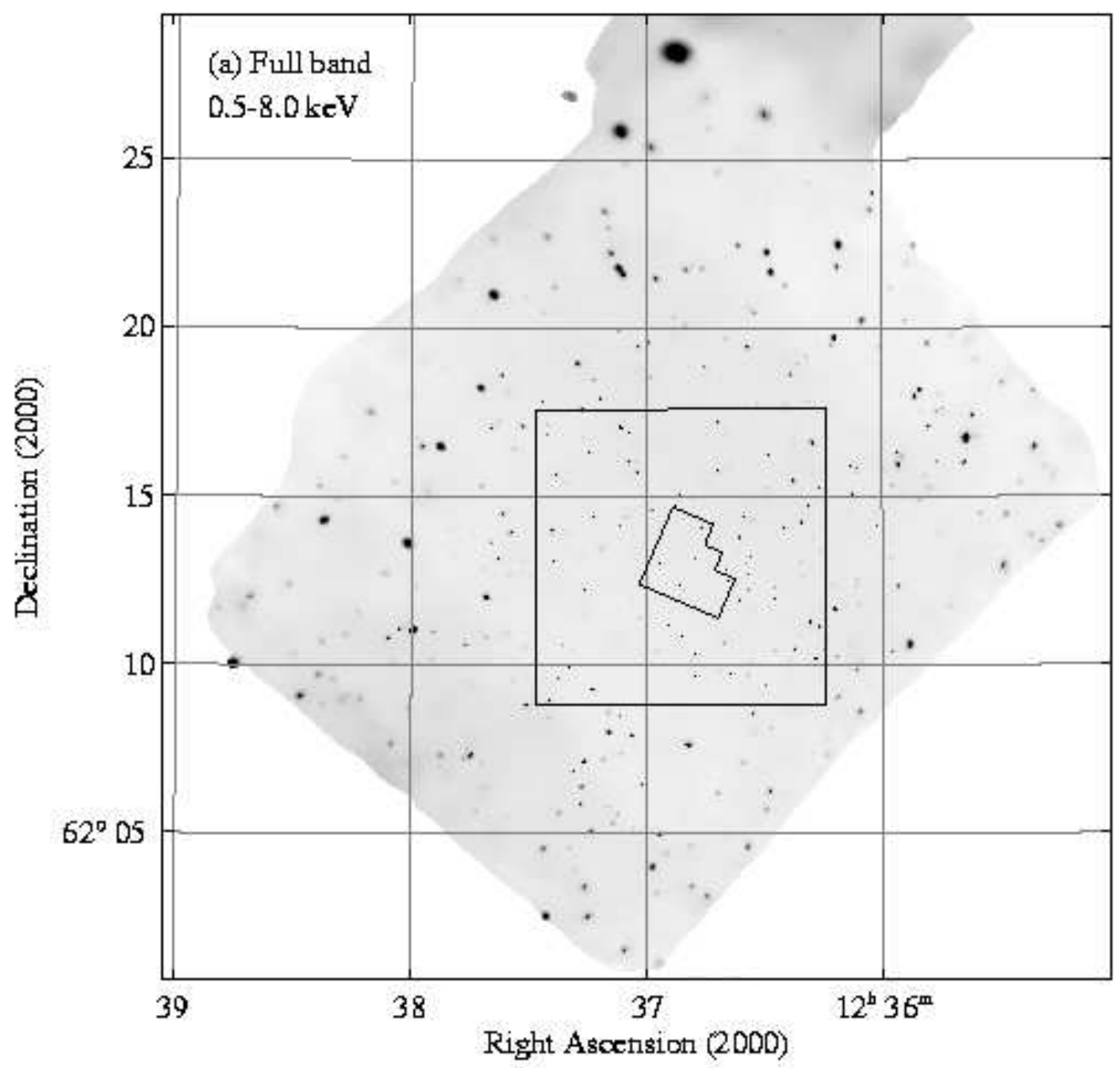

Fig. 4.- Adaptively smoothed and exposure-map corrected images of the CDF-N in the (a) full band, (b) soft band, (c) hard band, and (d) ultrahard band. These images have been made using the standard ASCA grade set (see Table 2), and they are binned by a factor of four in both right ascension and declination. The adaptive smoothing has been performed using the code of Ebeling, White, \& Rangarajan (2001) at the $2.5 \sigma$ level, and the grayscales are linear. Much of the apparent diffuse emission is just instrumental background; see $\S 3.3$ for a discussion of extended sources. The edges of the image appear "rounded" due to the combination of the dither of Chandra and the requirement that the effective exposure time exceed $50 \mathrm{ks}$ (see $§ 3.1$ ). The small polygon indicates the HDF-N, and the large square indicates the Caltech area. Note: Only one of the four pages of images could be included here; please see the World Wide Web site listed in Footnote 13 for the version with all the images. 


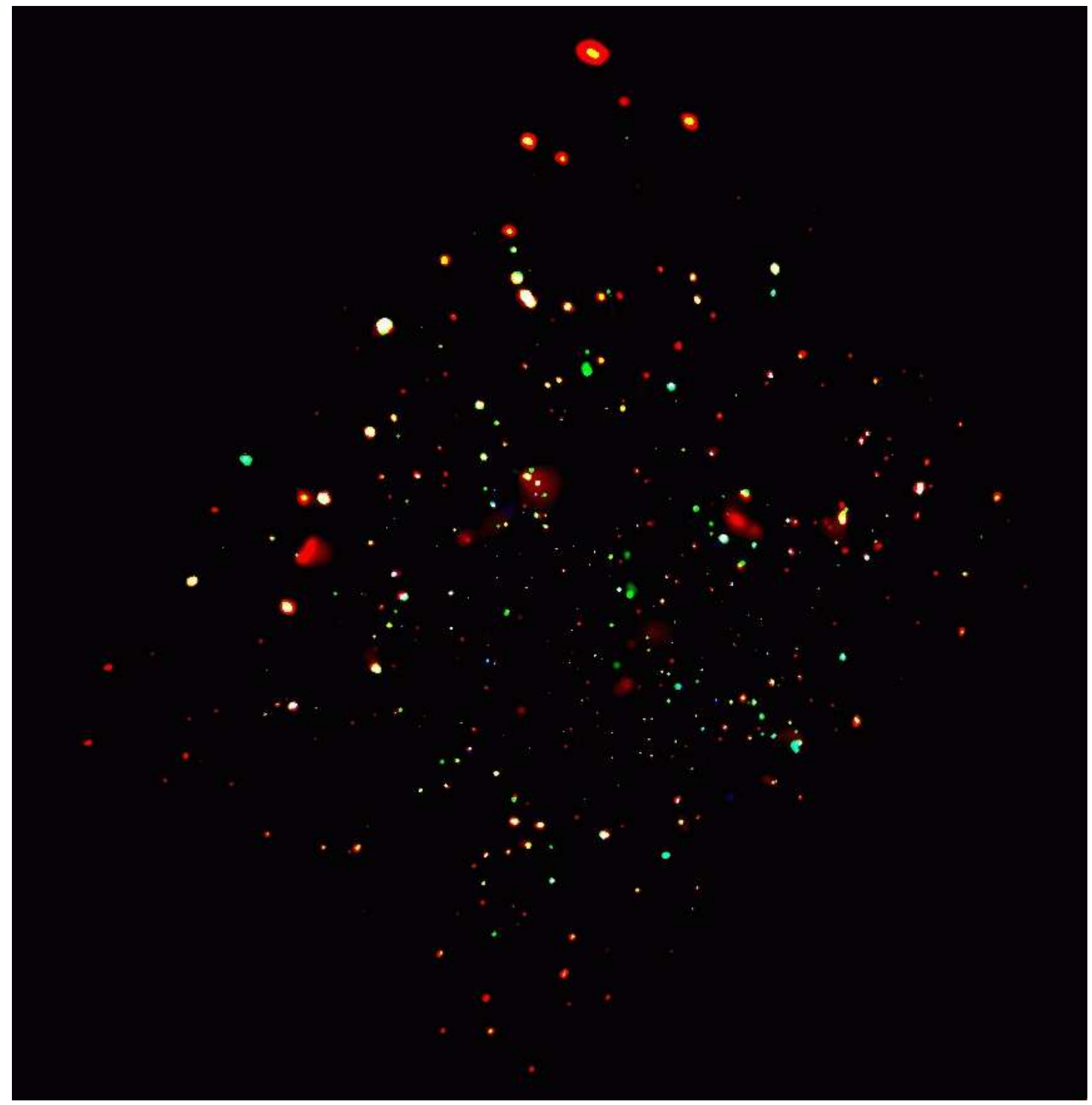

Fig. 5.- Chandra "true-color" image of the CDF-N. This image has been constructed from the soft-band (red), hard-band (green), and ultrahard-band (blue) images shown in Figure 4. Two of the red diffuse patches are CXOHDFN J123620.0+621554 and CXOHDFN J123756.0+621506 (see $\S 3.3)$. 


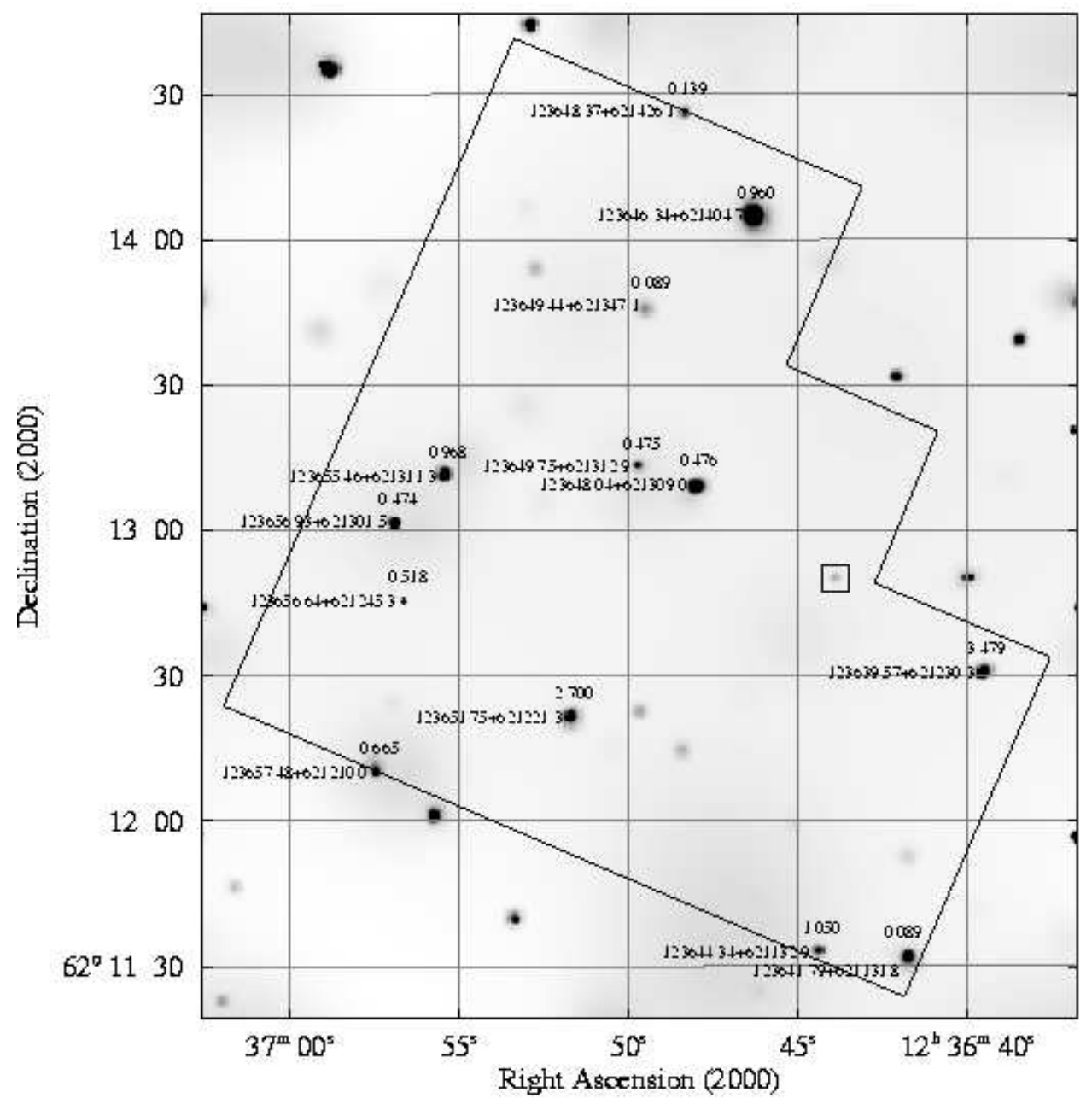

Fig. 6.- Adaptively smoothed Chandra image of the HDF-N and its immediate environs in the full band. This image has been made using the standard $A S C A$ grade set, and it has not been binned. The adaptive smoothing has been performed using the code of Ebeling et al. (2001) at the $2.5 \sigma$ level, and the grayscales are linear. Sources detected in this paper are labeled; labels are given immediately to the left of the corresponding X-ray sources, and redshifts are given immediately above the corresponding X-ray sources. The unlabeled enhancements apparent in the HDF-N are discussed in §3.2.3. One of these, CXOHDFN J123643.9+621249, is boxed (this is the variable source detected in Paper IV that is not detected here). 


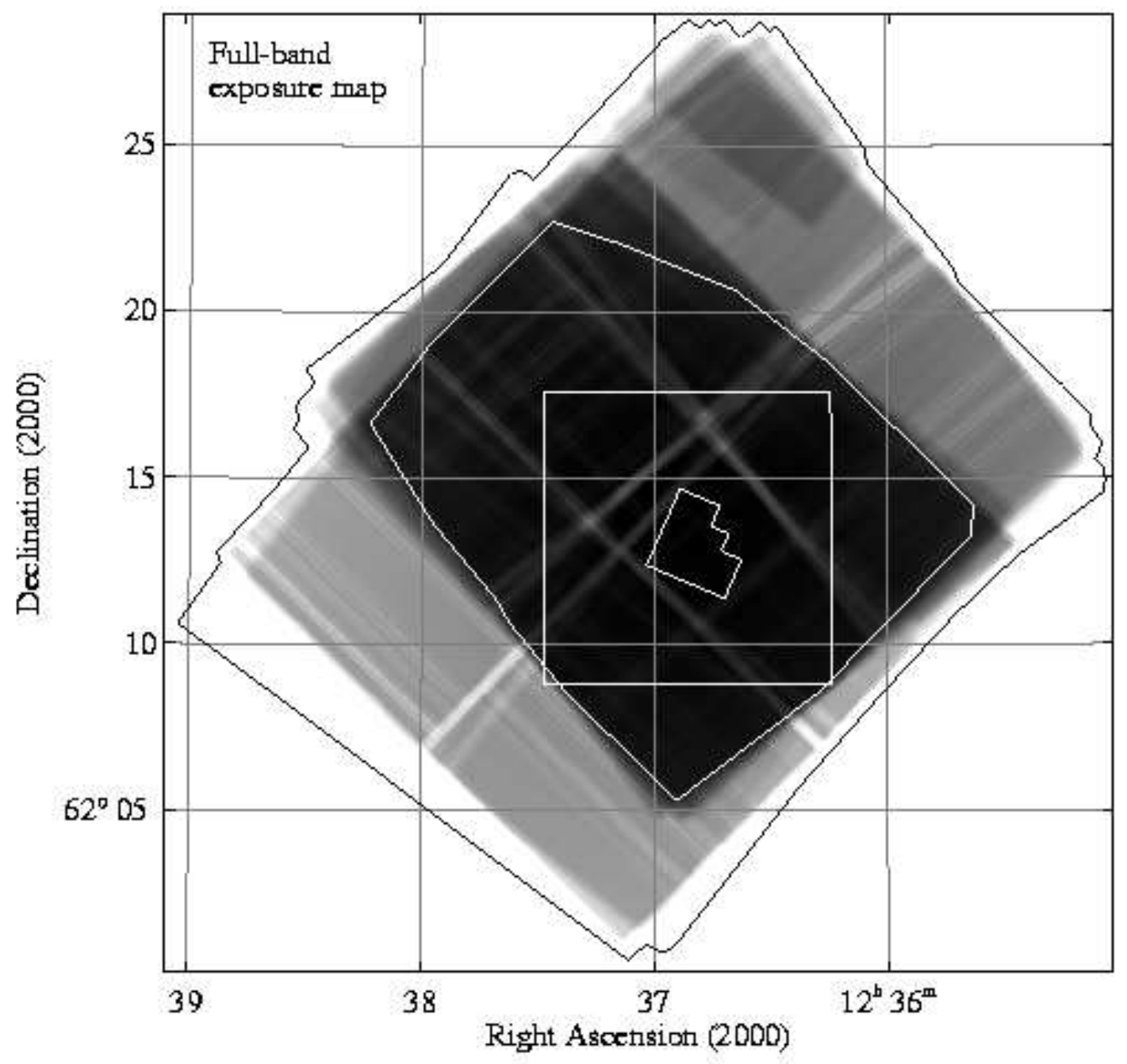

Fig. 7.- Combined full-band exposure map for the CDF-N ACIS observations. The darkest areas correspond to the highest effective exposure times (the maximum value is $948.7 \mathrm{ks}$ ), and the grayscales are logarithmic. The light grooves running through the exposure map correspond to the gaps between the CCDs and bad columns. The small white polygon indicates the HDF-N itself, the large white square indicates the Caltech area, and the large white polygon indicates a "high-exposure area" where the median exposure time is $>800 \mathrm{ks}$. The black outline surrounding the exposure map indicates the extent of all the ACIS-I observations; the regions of the exposure map near the outline appear white due to low exposure (20-200 ks). 


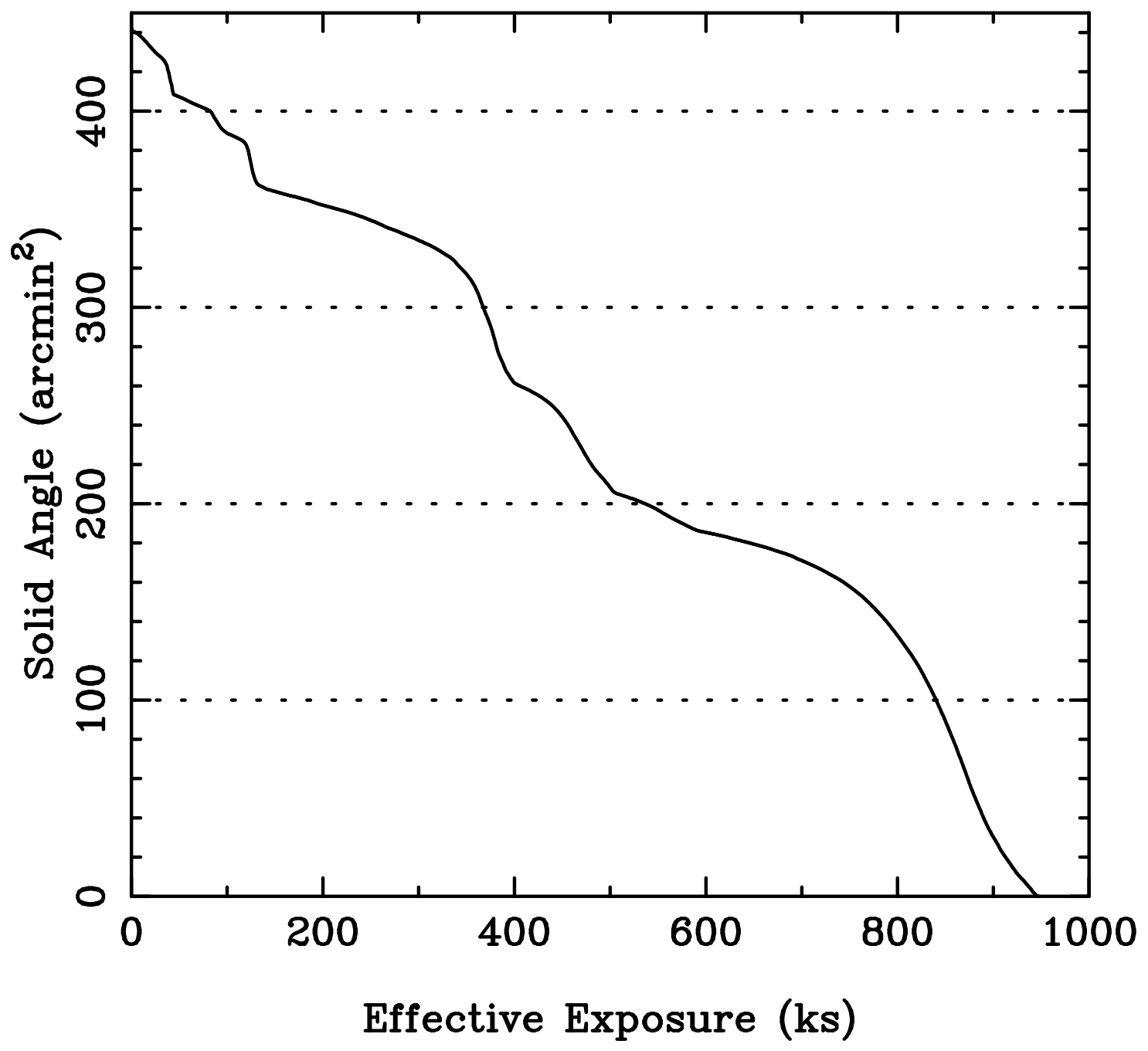

Fig. 8.- Amount of survey solid angle having at least a given amount of effective exposure in the full-band exposure map. Compare with Figure 7. 


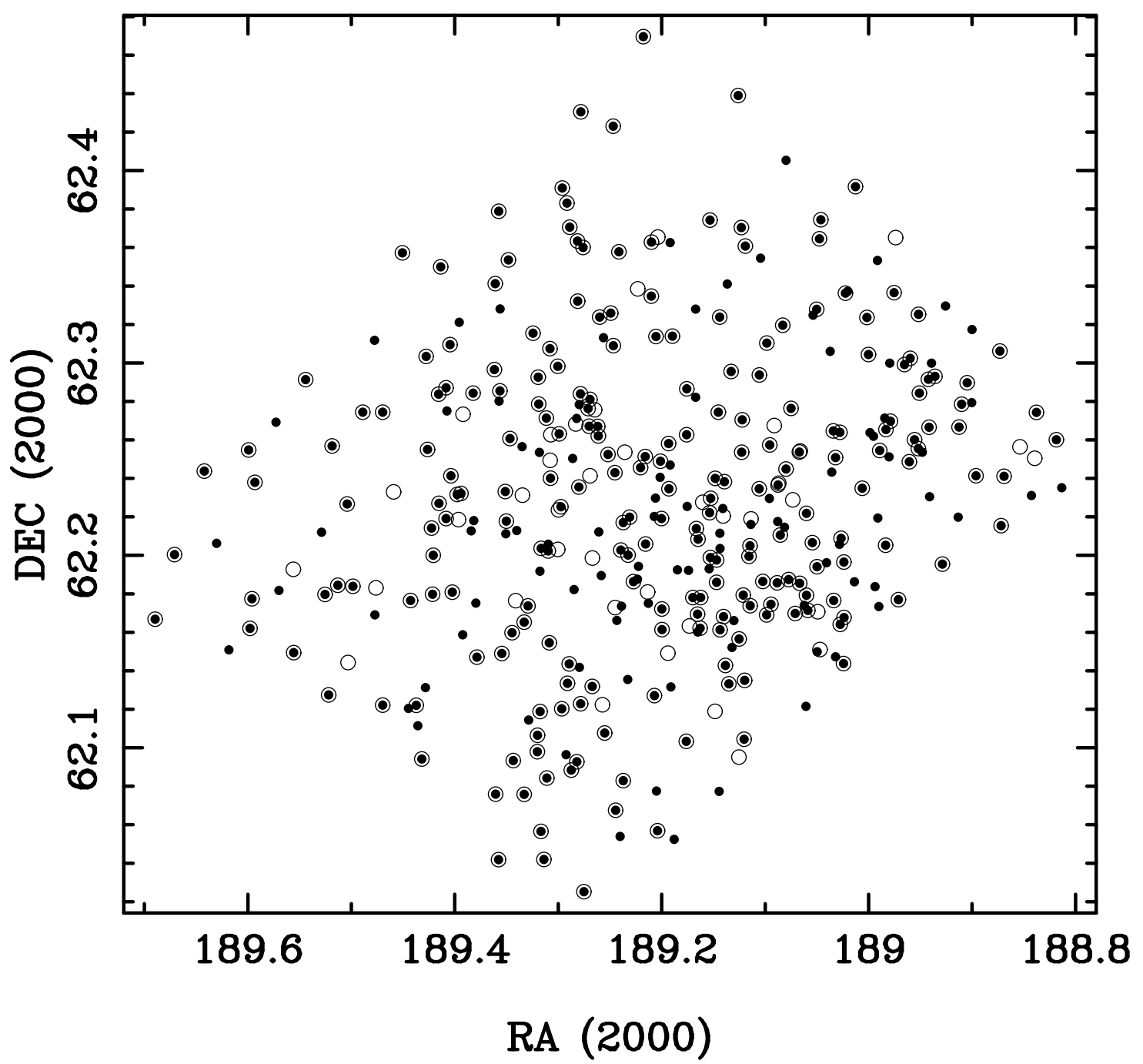

Fig. 9.- Positions of the soft-band (solid dots) and hard-band (circles) sources in Table 3. This plot removes the illusory effect of the changing PSF size across the field of view (although, of course, the detected source density is still modulated by the varying PSF size and effective exposure time). 


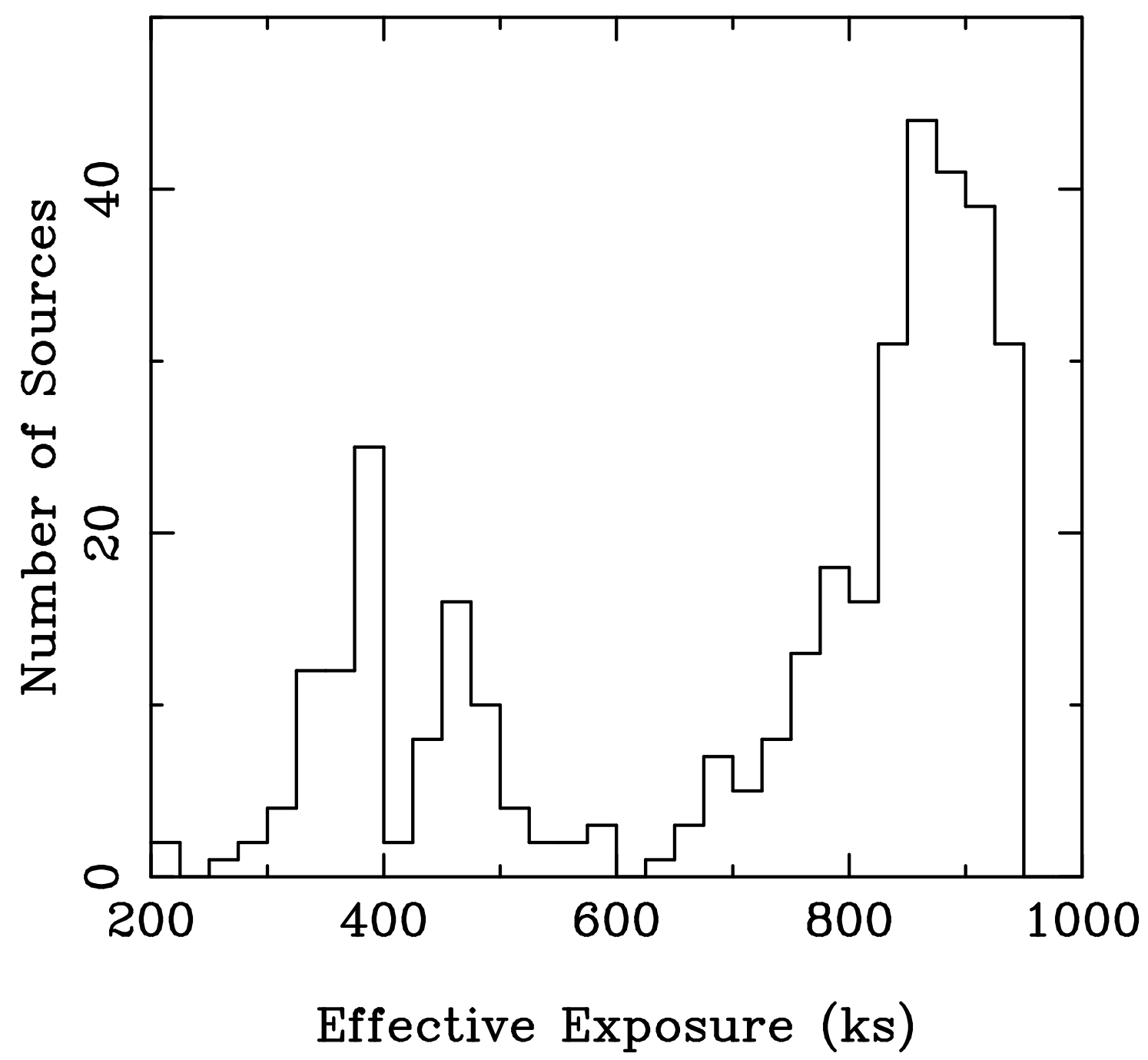

Fig. 10.- Histogram showing the distribution of full-band effective exposure time for the sources in Table 3. Most of the sources have $>600 \mathrm{ks}$ exposures, but a significant number have 200-600 ks exposures. 


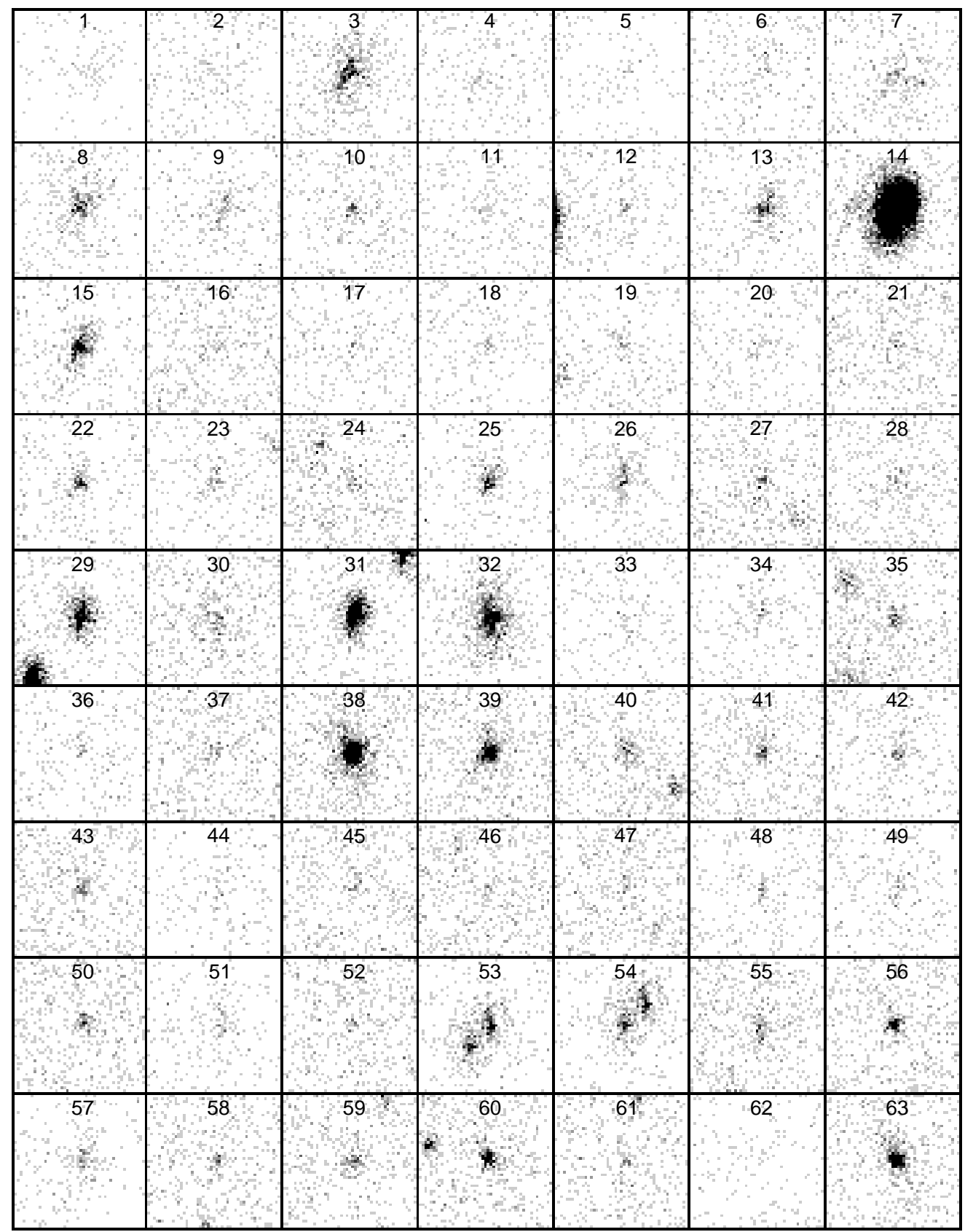

Fig. 11.- Full-band "postage-stamp" images for the Chandra sources in Table 3; the sources are numbered as in Table 3. Each image is oriented so that North is up and East is to the left, and each is 50 pixels $\left(\approx 244^{\prime \prime} 6\right)$ on a side. The source of interest is always at the center of the image. The background varies significantly from image to image due to the varying effective exposure time (see Figure 7). In a few cases no source is apparent; these sources were not detected in the full band but were detected in one of the other bands. A few of the sources appear to show extent; in most cases this is an artifact of the complex Chandra off-axis PSF. Note: Only one of the six pages of cutouts could be included here; please see the World Wide Web site listed in Footnote 13 for the version with all the cutouts. 


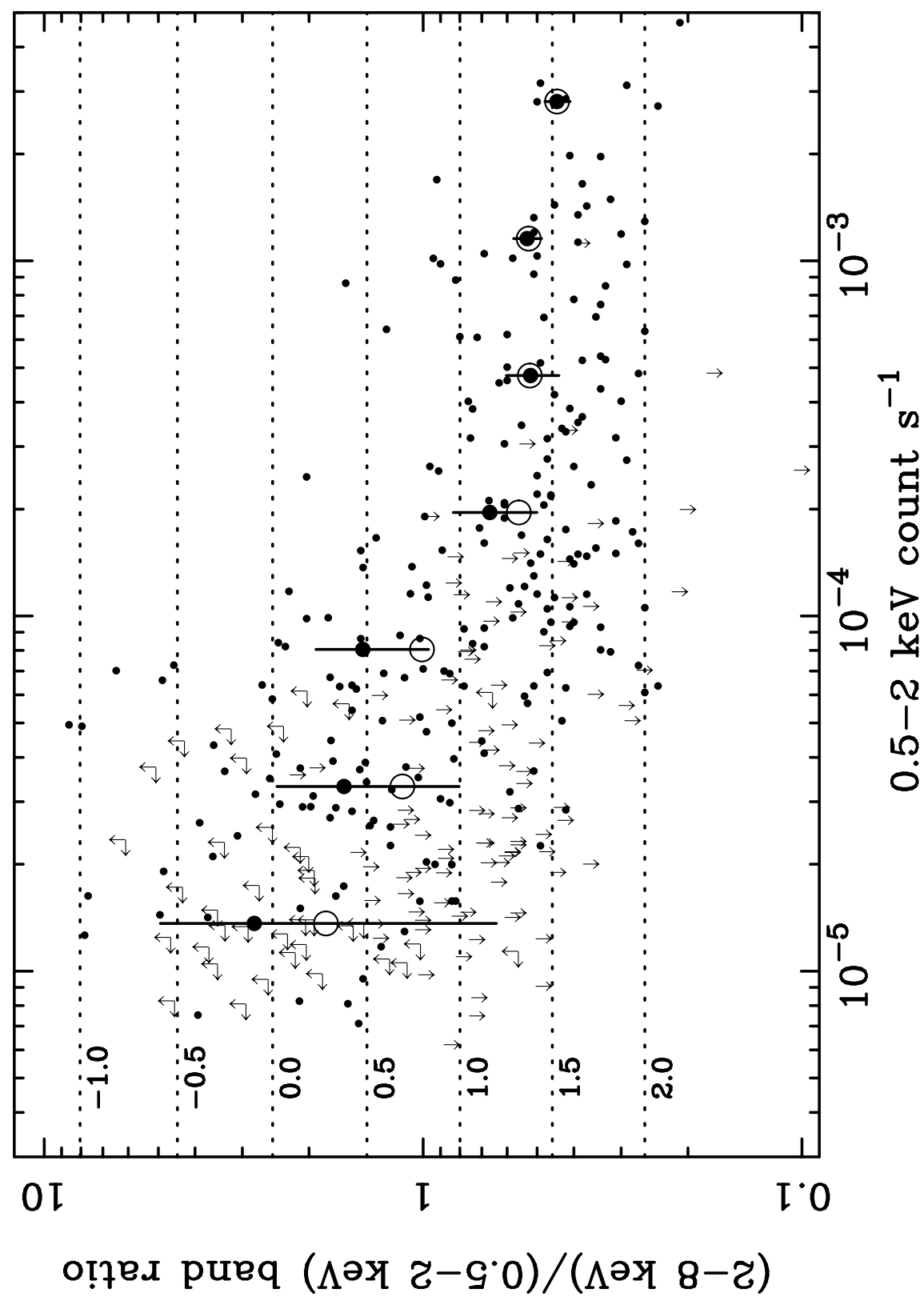

Fig. 12.- Band ratio as a function of soft-band count rate for the sources in Table 3. Small solid dots show sources detected in both the soft and hard bands, and plain arrows show sources detected in only one of these two bands (sources detected in only the full band are not plotted). To reduce symbol crowding, we do not show error bars for each of the small solid dots. Instead, the large solid dots show average band ratios and error bars for the small solid dots as a function of soft-band count rate (these large solid dots are given only to show the size of the errors, and they should not be interpreted statistically). The open circles show average band ratios derived from stacking analyses (following $\S 3.3$ of Paper VI). Horizontal dotted lines are labeled with the photon indices that correspond to a given band ratio assuming only Galactic absorption (these were determined using the CXC's Portable, Interactive, Multi-Mission Simulator). 


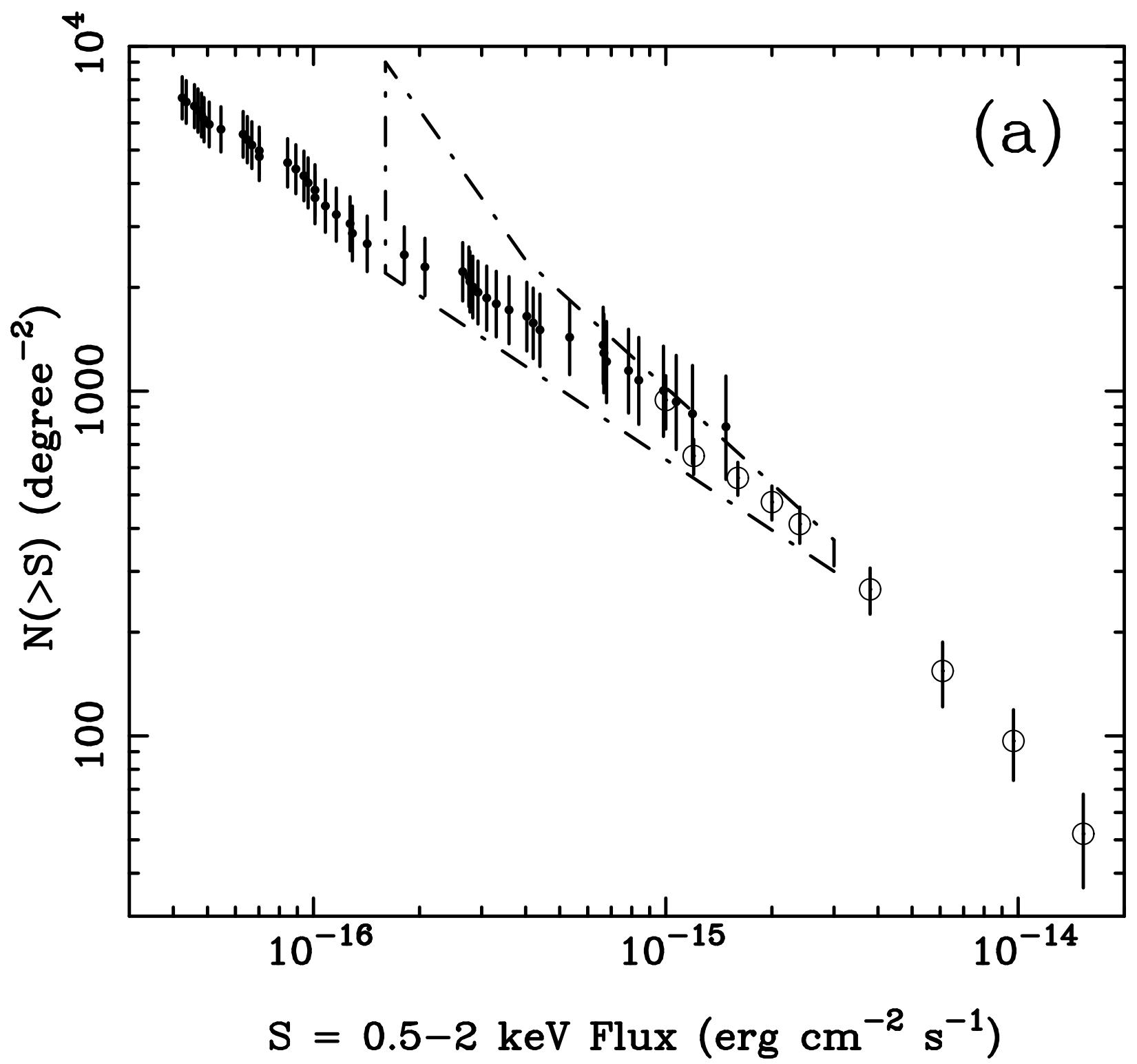

Fig. 13.- Number of sources, $N(>S)$, brighter than a given flux, $S$, for the (a) soft band and (b) hard band. In panel (a) the solid dots are our data points, the open circles are the ROSAT Lockman Hole data points from Hasinger et al. (1998), and the dot-dashed "bow-tie" region shows the ROSAT Lockman Hole fluctuation analysis results of Hasinger et al. (1993). In panel (b) the solid dots are our data points, the open circles are the $A S C A$ Large Sky Survey and Deep Sky Survey data points from Ueda et al. (1998) and Ogasaka et al. (1998), and the dot-dashed bow-tie region shows the multiple-field fluctuation analysis results of Gendreau, Barcons, \& Fabian (1998). The Ueda et al. (1998), Ogasaka et al. (1998), and Gendreau et al. (1998) results have been corrected to the $2-8 \mathrm{keV}$ band assuming a $\Gamma=1.4$ power-law spectrum. The error bars on our data points have been computed following Gehrels (1986) for $1 \sigma$. We have only plotted data points where more than 10 sources were used to determine the number counts. 


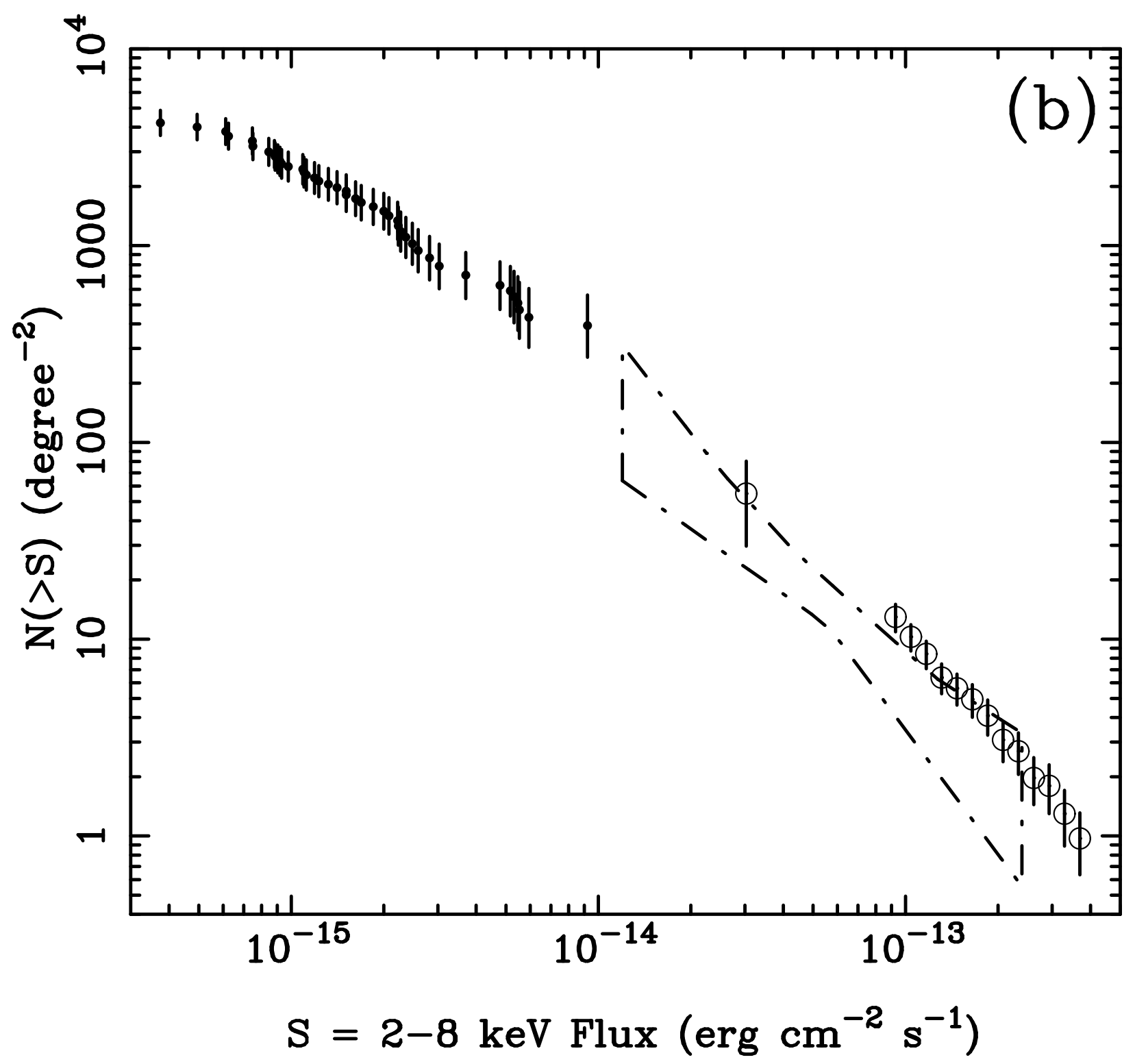




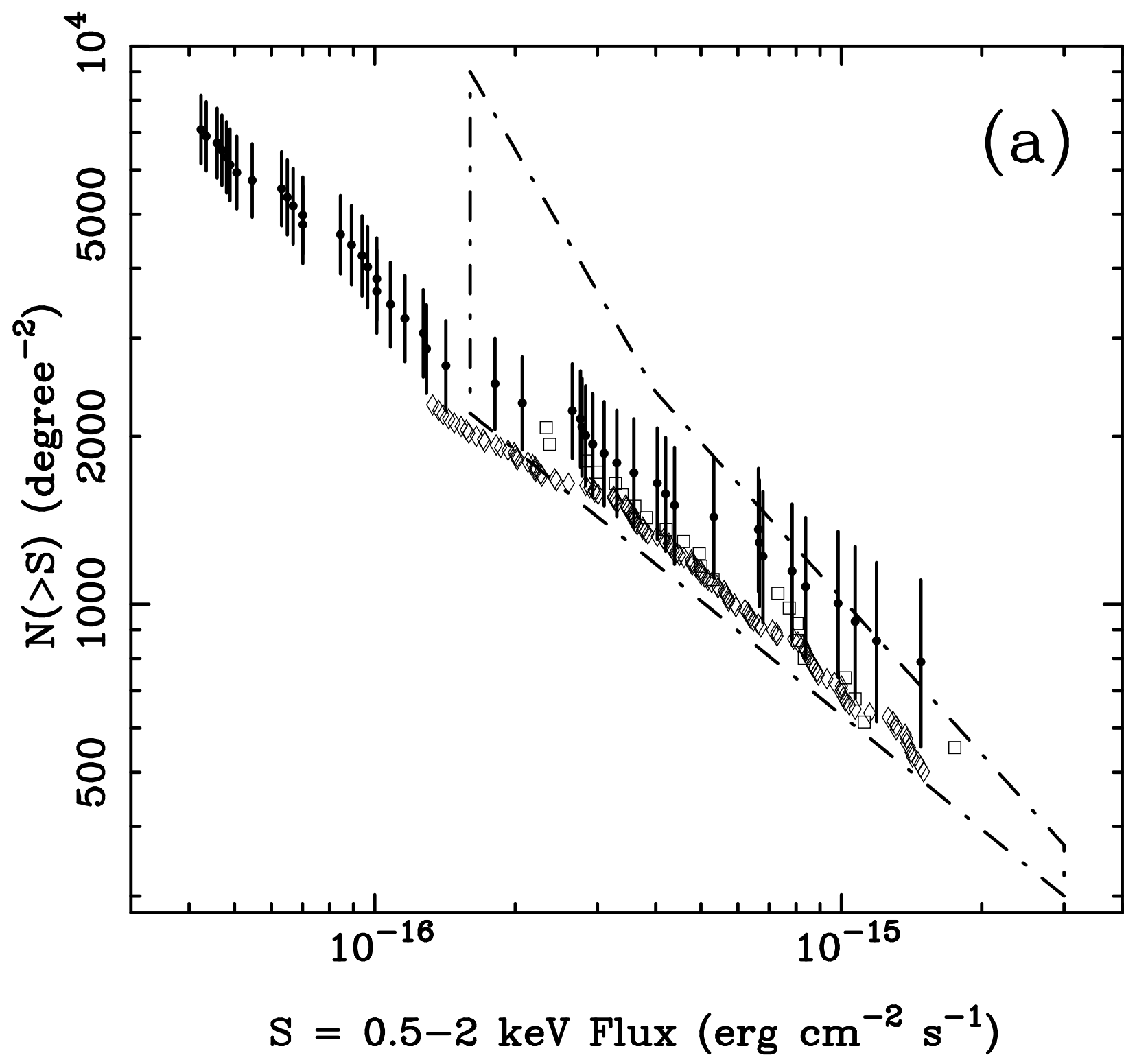

Fig. 14. - Comparisons of our $N(>S$ ) curves (solid dots) with those from Mushotzky et al. (2000; open squares) and Tozzi et al. (2001; open diamonds) for the (a) soft band and (b) hard band. The dot-dashed regions show the fluctuation analysis results described in Figure 13. In panel (b) the Mushotzky et al. (2000) and Tozzi et al. (2001) results have been corrected to the $2-8 \mathrm{keV}$ band assuming a $\Gamma=1.4$ power-law spectrum. 


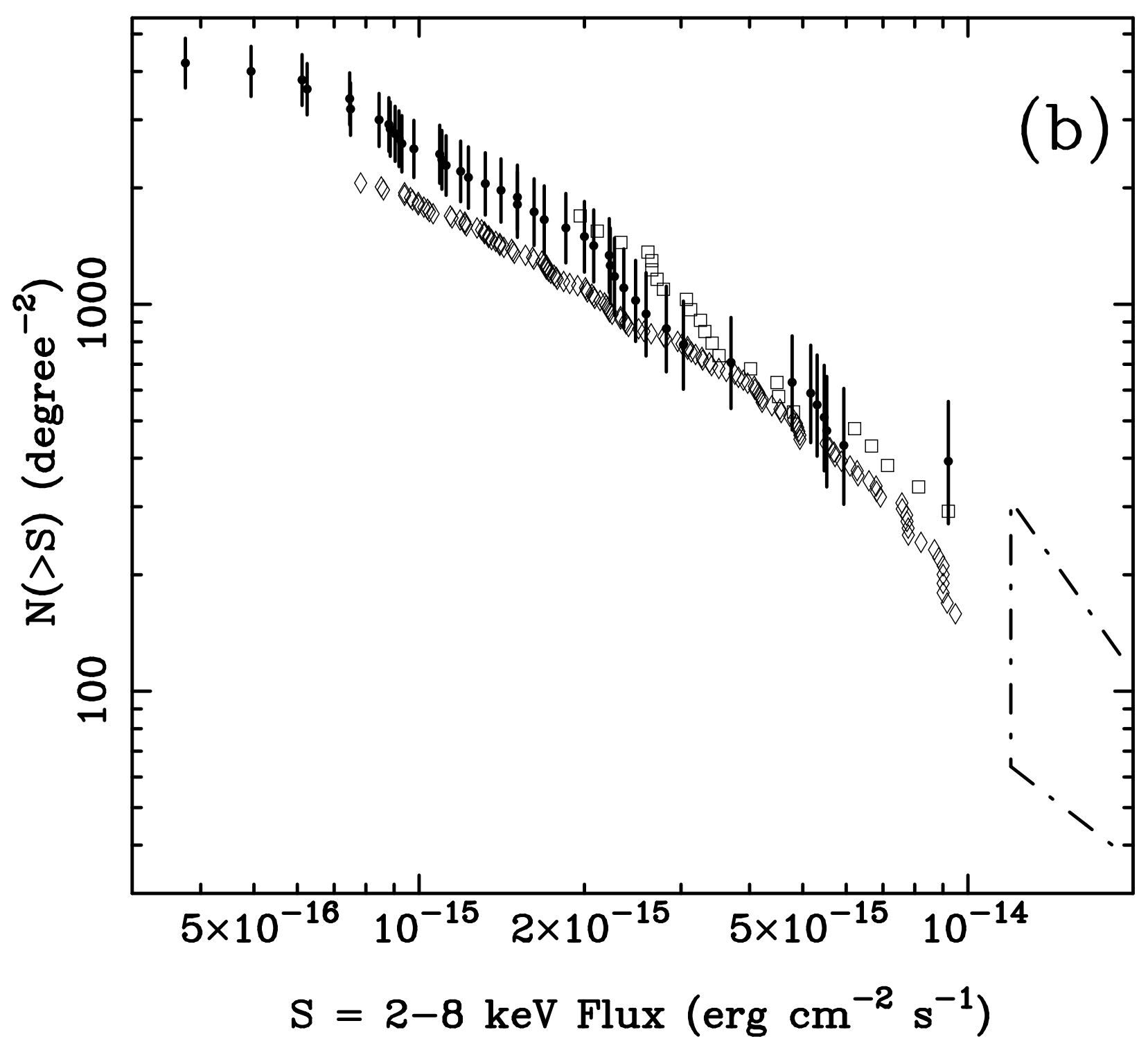



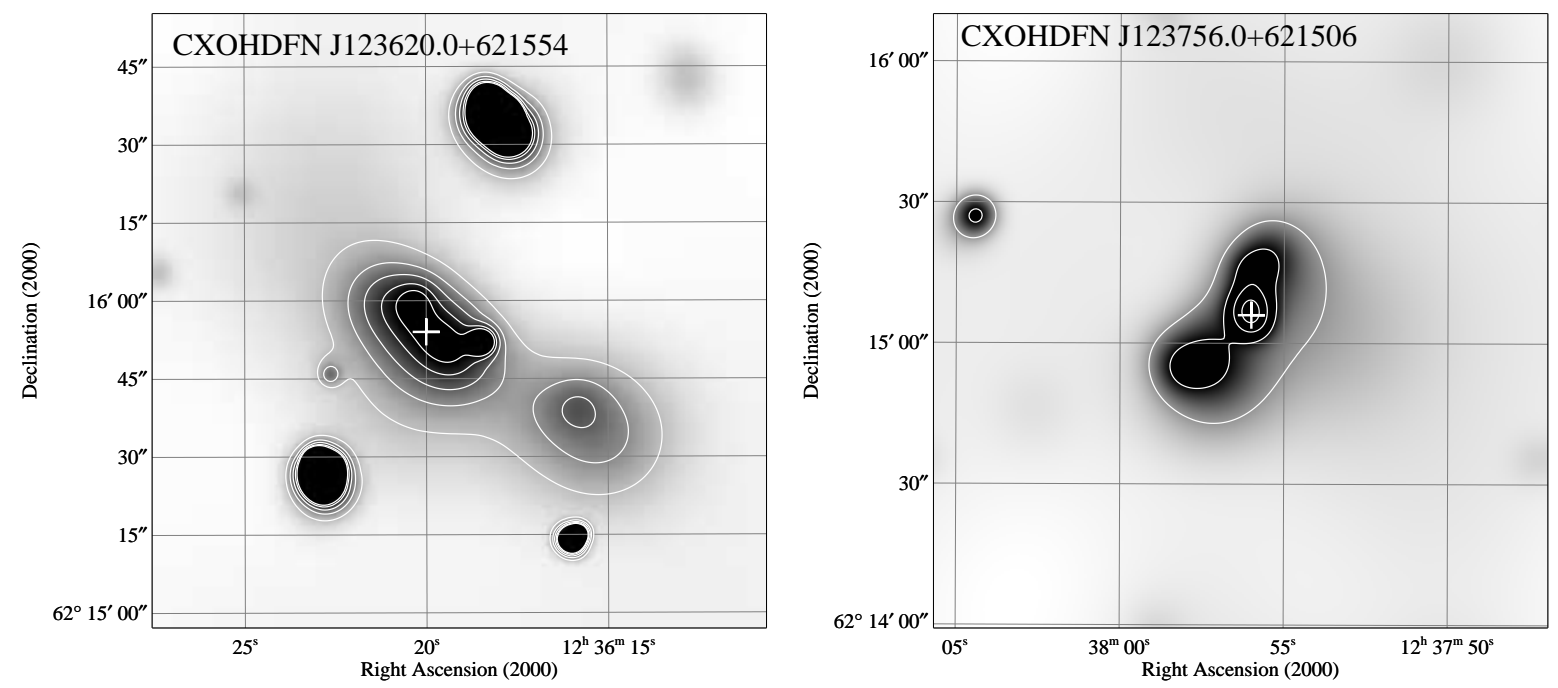

Fig. 15. - Adaptively smoothed and contoured Chandra soft-band images of the spatially extended X-ray sources CXOHDFN J123620.0+621554 (contours are at 55, 65, 75, 85, and 95\% of the maximum pixel value) and CXOHDFN J123756.0+621506 (contours are at 30, 50, 70, and 90\% of the maximum pixel value). The adaptive smoothing has been performed using the code of Ebeling et al. (2001) at the $2.5 \sigma$ level, and the grayscales are linear. A cross indicates the adopted "central" position of each extended source. The point sources in each image can be used to judge the appropriate PSF sizes. 


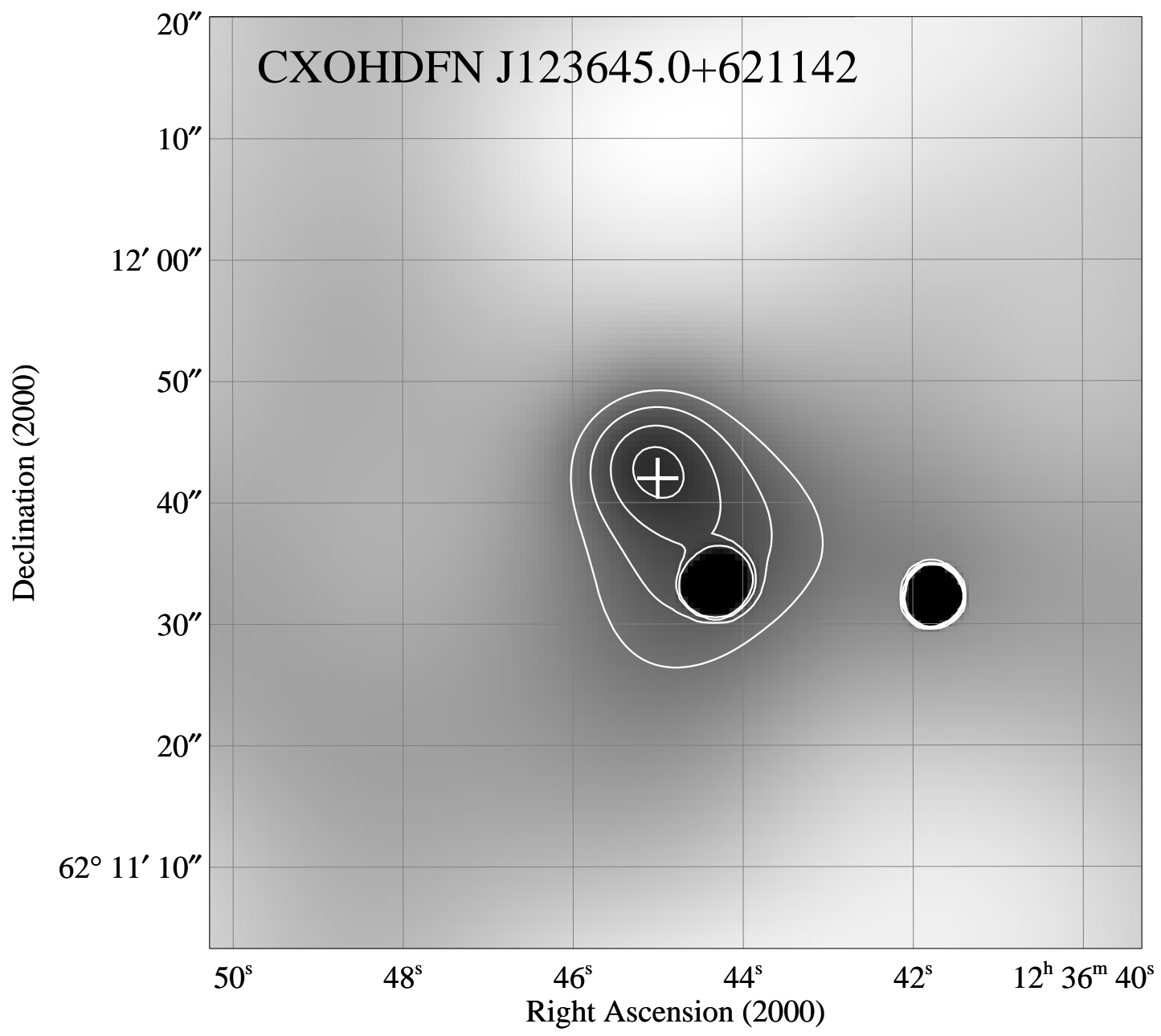

Fig. 16. - Adaptively smoothed and contoured Chandra soft-band image of the probable spatially extended X-ray source CXOHDFN J123645.0+621142 (contours are at 85, 90, 95, and 99\% of the maximum pixel value). The adaptive smoothing has been performed using the code of Ebeling et al. (2001) at the $2.5 \sigma$ level, and the grayscales are linear. A cross indicates the adopted "central" position of the extended source. The point sources in the image can be used to judge the appropriate PSF size. The point source within the diffuse emission, CXOHDFN J123644.3+621132, is associated with the $z=1.050 \mathrm{FR}$ I radio galaxy VLA J123644.3+621133. 
$-1-$ 
Table 3. Main Chandra Point-Source Catalog

\begin{tabular}{|c|c|c|c|c|c|c|c|c|c|c|c|c|c|c|c|}
\hline \multirow[b]{2}{*}{$\begin{array}{c}\# \\
(1)\end{array}$} & \multicolumn{2}{|c|}{ Coordinates } & \multirow{2}{*}{$\begin{array}{l}\text { Positional } \\
\text { Error } \\
(4)\end{array}$} & \multicolumn{4}{|c|}{ Counts } & \multirow{2}{*}{$\begin{array}{l}\text { Band } \\
\text { Ratio } \\
(9)\end{array}$} & \multirow{2}{*}{$\begin{array}{c}\text { Effective } \\
\Gamma \\
(10)\end{array}$} & \multirow{2}{*}{$\begin{array}{c}\text { Exposure } \\
\text { Exposure }(\mathrm{ks}) \\
(11)\end{array}$} & \multirow[b]{2}{*}{$\begin{array}{l}\text { FB } \\
(12)\end{array}$} & \multicolumn{3}{|c|}{ Flux } & \multirow[b]{2}{*}{$\begin{array}{l}\text { Notes } \\
(16)\end{array}$} \\
\hline & $\begin{array}{c}\alpha_{2000} \\
(2)\end{array}$ & $\begin{array}{c}\delta_{2000} \\
(3)\end{array}$ & & $\begin{array}{l}\mathrm{FB} \\
(5)\end{array}$ & $\begin{array}{l}\text { SB } \\
(6)\end{array}$ & $\begin{array}{l}\mathrm{HB} \\
(7)\end{array}$ & $\begin{array}{l}\text { UHB } \\
(8)\end{array}$ & & & & & $\begin{array}{l}\text { SB } \\
(13)\end{array}$ & $\begin{array}{l}\mathrm{HB} \\
(14)\end{array}$ & $\begin{array}{l}\text { UHB } \\
(15)\end{array}$ & \\
\hline 1 & $12 \quad 35 \quad 15.33$ & +621406.9 & 1.3 & $52.3 \pm 9.6$ & $39.7 \pm 7.3$ & $<20.6$ & $<16.6$ & $<0.53$ & $>1.41$ & 130.4 & 4.09 & 1.36 & $<3.41$ & $<4.72$ & $\mathrm{M}$ \\
\hline 2 & 123516.56 & +621536.9 & 1.3 & $59.6 \pm 15.8$ & $30.1 \pm 10.5^{\mathrm{b}}$ & $29.1 \pm 12.7$ & $<24.5$ & $1.00_{-0.51}^{+0.69}$ & 0.80 & 422.4 & 2.01 & 0.31 & 1.70 & $<2.33$ & \\
\hline 3 & $1235 \quad 21.17$ & +621628.2 & 1.2 & $470.1 \pm 26.8$ & $311.2 \pm 20.6$ & $143.2 \pm 17.1$ & $40.2 \pm 11.6^{\mathrm{b}}$ & $0.48_{-0.06}^{+0.07}$ & 1.50 & 448.2 & 10.13 & 3.10 & 6.75 & 3.28 & \\
\hline 4 & 123521.55 & +621502.3 & 1.2 & $55.3 \pm 14.4^{\mathrm{b}}$ & $<24.6$ & $39.3 \pm 12.3^{\mathrm{a}}$ & $<25.8$ & $>1.65$ & $<0.30$ & 432.1 & 2.36 & $<0.25$ & 2.51 & $<2.53$ & \\
\hline 5 & $12 \quad 35 \quad 22.34$ & $+6213 \quad 52.5$ & 1.2 & $32.4 \pm 10.8^{\mathrm{bc}}$ & $24.5 \pm 8.0$ & $<19.6$ & $<15.7$ & $<0.83$ & $1.40^{\mathrm{d}}$ & 196.2 & 1.69 & 0.55 & $<2.15$ & $<2.93$ & $\mathrm{O}$ \\
\hline 6 & $1235 \quad 24.99$ & +621523.7 & 1.1 & $53.6 \pm 14.6$ & $<22.2$ & $52.9 \pm 13.0$ & $<26.6$ & $>2.46$ & $<-0.01$ & 451.8 & 2.55 & $<0.21$ & 3.47 & $<2.58$ & NP \\
\hline 7 & $1235 \quad 28.68$ & +621428.6 & 1.1 & $168.1 \pm 17.9$ & $70.7 \pm 11.5$ & $100.4 \pm 14.7$ & $38.1 \pm 10.7$ & $1.46_{-0.29}^{+0.35}$ & 0.42 & 461.2 & 6.33 & 0.67 & 5.81 & 3.39 & \\
\hline 8 & $1235 \quad 29.36$ & +621255.9 & 1.1 & $272.7 \pm 20.4$ & $197.3 \pm 16.4$ & $73.6 \pm 12.6$ & $29.8 \pm 9.3^{\mathrm{bc}}$ & $0.38_{-0.07}^{+0.07}$ & 1.66 & 377.9 & 6.35 & 2.36 & 3.96 & 2.83 & $\mathrm{O}$ \\
\hline 9 & $12 \quad 3529.64$ & +621822.9 & 1.2 & $125.0 \pm 16.9$ & $60.1 \pm 11.4$ & $62.3 \pm 13.2$ & $<25.0$ & $\begin{array}{l}1.07_{-0.28}^{+0.34} \\
\end{array}$ & 0.74 & 434.6 & 4.25 & 0.60 & 3.58 & $<2.29$ & NP \\
\hline 10 & 123535.16 & +621429.4 & 1.0 & $96.2 \pm 15.3$ & $60.6 \pm 10.9$ & $33.1 \pm 11.4^{\mathrm{a}}$ & $<21.3$ & $0.56_{-0.21}^{+0.23}$ & 1.37 & 558.6 & 1.80 & 0.48 & 1.28 & $<1.38$ & \\
\hline 11 & 123536.10 & +621903.2 & 1.2 & $59.6 \pm 14.4$ & $44.7 \pm 10.5$ & $<28.5$ & $<21.7$ & $<0.66$ & $>1.22$ & 461.2 & 1.46 & 0.43 & $<1.38$ & $<1.76$ & \\
\hline 12 & 123536.17 & +621646.3 & 1.0 & $41.1 \pm 13.2$ & $32.4 \pm 10.2$ & $<23.3$ & $<20.3$ & $<0.74$ & $>1.11$ & 426.4 & 1.16 & 0.33 & $<1.24$ & $<1.77$ & I \\
\hline 13 & $12 \quad 35 \quad 37.23$ & +621723.5 & 1.1 & $201.6 \pm 18.9$ & $146.6 \pm 14.9$ & $44.6 \pm 11.9$ & $<22.5$ & $0.31_{-0.09}^{+0.09}$ & 1.79 & 460.2 & 3.59 & 1.44 & 1.91 & $<1.68$ & N \\
\hline 14 & 123538.51 & +621643.4 & 1.0 & $9100.7 \pm 97.8$ & $6633.0 \pm 83.2$ & $2481.0 \pm 52.6$ & $792.0 \pm 31.3$ & $0.38_{-0.01}^{+0.01}$ & 1.66 & 430.5 & 186.76 & 68.84 & 116.53 & 63.66 & NP I \\
\hline 15 & 123539.09 & +621600.6 & 1.0 & $338.9 \pm 22.2$ & $246.4 \pm 18.1$ & $80.7 \pm 12.9$ & $23.9 \pm 8.9^{\mathrm{a}}$ & $0.33_{-0.06}^{+0.06}$ & 1.75 & 467.1 & 6.08 & 2.38 & 3.43 & 1.75 & \\
\hline 16 & 123539.28 & +621311.8 & 0.9 & $21.8 \pm 12.7^{\mathrm{a}}$ & $22.6 \pm 9.1^{\mathrm{a}}$ & $<24.6$ & $<21.6$ & $<1.11$ & $1.40^{\mathrm{d}}$ & 795.9 & 0.28 & 0.13 & $<0.66$ & $<0.98$ & \\
\hline 17 & 123542.24 & +621947.2 & 1.1 & $59.7 \pm 14.4^{\mathrm{b}}$ & $38.0 \pm 10.2^{\mathrm{c}}$ & $<28.2$ & $<22.1$ & $<0.76$ & $>1.08$ & 472.8 & 1.55 & 0.35 & $<1.37$ & $<1.77$ & $\mathrm{O}$ \\
\hline 18 & 123542.95 & +621143.8 & 0.9 & $33.9 \pm 10.7$ & $12.0 \pm 6.8^{\mathrm{b}}$ & $20.7 \pm 9.0$ & $<16.7$ & $1.76_{-1.00}^{+2.45}$ & 0.25 & 443.1 & 1.45 & 0.12 & 1.29 & $<1.58$ & \\
\hline 19 & 123544.67 & +621735.6 & 1.0 & $39.4 \pm 11.7$ & $13.3 \pm 7.7$ & $25.7 \pm 9.7^{\mathrm{bc}}$ & $<19.4$ & $1.98_{-1.84}^{+2.03}$ & 0.15 & 454.3 & 1.73 & 0.13 & 1.60 & $<1.78$ & $\mathrm{O}$ \\
\hline 20 & 123545.48 & +621800.2 & 1.0 & $36.6 \pm 11.7$ & $30.6 \pm 9.2$ & $<18.9$ & $<14.9$ & $<0.63$ & $>1.26$ & 478.6 & 0.85 & 0.28 & $<0.87$ & $<1.13$ & \\
\hline 21 & 123545.91 & +621350.1 & 0.9 & $39.9 \pm 12.3^{\mathrm{a}}$ & $21.9 \pm 8.3$ & $<24.5$ & $<20.4$ & $<1.14$ & $1.40^{\mathrm{d}}$ & 840.8 & 0.49 & 0.12 & $<0.62$ & $<0.86$ & \\
\hline 22 & 123546.03 & +621600.1 & 0.9 & $114.4 \pm 14.8$ & $55.5 \pm 10.1$ & $53.6 \pm 11.1$ & $<18.4$ & $0.98_{-0.25}^{+0.30}$ & 0.82 & 452.8 & 3.56 & 0.53 & 2.85 & $<1.54$ & \\
\hline 23 & 123546.19 & +621729.8 & 1.0 & $61.3 \pm 12.6$ & $43.0 \pm 9.7$ & $17.1 \pm 9.0^{\mathrm{b}}$ & $<16.8$ & $0.41_{-0.23}^{+0.24}$ & 1.62 & 457.8 & 1.21 & 0.42 & 0.76 & $<1.27$ & \\
\hline 24 & 123547.63 & +621513.5 & 0.8 & $54.2 \pm 12.8$ & $34.2 \pm 9.3$ & $<24.0$ & $<17.5$ & $<0.72$ & $>1.14$ & 775.3 & 0.83 & 0.19 & $<0.70$ & $<0.83$ & NP \\
\hline 25 & 123548.30 & +621703.9 & 0.9 & $166.0 \pm 16.6$ & $124.7 \pm 13.5$ & $35.7 \pm 10.0$ & $13.1 \pm 7.6^{\mathrm{b}}$ & $0.29_{-0.09}^{+0.09}$ & 1.83 & 453.1 & 2.93 & 1.25 & 1.53 & 0.97 & \\
\hline 26 & $12 \quad 35 \quad 48.47$ & +621931.5 & 1.1 & $164.4 \pm 17.6$ & $101.6 \pm 12.9$ & $66.6 \pm 12.8$ & $<23.6$ & $0.67_{-0.15}^{+0.16}$ & 1.20 & 479.1 & 3.93 & 0.93 & 3.10 & $<1.82$ & \\
\hline 27 & 123548.52 & $+6215 \quad 20.2$ & 0.8 & $70.2 \pm 13.4$ & $28.5 \pm 8.8^{\mathrm{a}}$ & $41.4 \pm 11.0$ & $<21.9$ & $1.47_{-0.52}^{+0.77}$ & 0.41 & 774.2 & 1.58 & 0.16 & 1.42 & $<1.15$ & \\
\hline 28 & 123549.40 & +621536.8 & 0.8 & $52.3 \pm 12.2$ & $24.7 \pm 8.4^{\mathrm{b}}$ & $26.9 \pm 9.9$ & $15.3 \pm 8.3^{\mathrm{b}}$ & $1.11_{-0.50}^{+0.70}$ & 0.70 & 656.7 & 1.20 & 0.16 & 1.02 & 0.91 & \\
\hline 29 & $1235 \quad 50.41$ & +621809.1 & 0.9 & $547.8 \pm 27.0$ & $297.8 \pm 19.6$ & $234.9 \pm 18.6$ & $107.4 \pm 13.3$ & $0.80_{-0.08}^{+0.09}$ & 1.03 & 485.6 & 14.22 & 2.69 & 11.16 & 8.20 & \\
\hline 30 & 123550.60 & +621455.5 & 0.8 & $109.4 \pm 14.8$ & $45.7 \pm 9.7^{\mathrm{c}}$ & $68.9 \pm 12.2$ & $39.1 \pm 10.0$ & $1.54_{-0.38}^{+0.50}$ & 0.37 & 839.1 & 2.32 & 0.24 & 2.20 & 1.89 & $\mathrm{O}$ \\
\hline 31 & $1235 \quad 51.74$ & +621757.3 & 0.9 & $914.3 \pm 33.9$ & $587.9 \pm 26.5$ & $297.3 \pm 20.3$ & $108.0 \pm 13.3$ & $0.51_{-0.04}^{+0.04}$ & 1.44 & 487.2 & 18.81 & 5.36 & 12.87 & 7.78 & NP \\
\hline 32 & 123553.15 & +621037.5 & 0.9 & $734.9 \pm 30.4$ & $548.4 \pm 25.5$ & $199.6 \pm 17.1$ & $71.3 \pm 11.1$ & $0.37_{-0.04}^{+0.04}$ & 1.68 & 383.3 & 16.70 & 6.43 & 10.43 & 6.45 & \\
\hline 33 & $1235 \quad 53.77$ & +622155.2 & 1.2 & $75.8 \pm 14.0$ & $<19.9$ & $66.6 \pm 12.6$ & $<26.4$ & $>3.38$ & $<-0.23$ & 418.4 & 4.31 & $<0.21$ & 4.93 & $<2.84$ & \\
\hline 34 & 123554.16 & +622011.9 & 1.0 & $76.2 \pm 13.9$ & $55.0 \pm 10.4$ & $19.8 \pm 10.0^{\mathrm{bc}}$ & $<20.0$ & $0.37_{-0.20}^{+0.21}$ & 1.69 & 475.2 & 1.40 & 0.52 & 0.84 & $<1.46$ & $\mathrm{O}$ \\
\hline 35 & 123555.01 & +621611.5 & 0.8 & $85.3 \pm 13.4$ & $22.4 \pm 8.2^{\mathrm{b}}$ & $61.0 \pm 11.6$ & $18.3 \pm 8.2^{\mathrm{b}}$ & $2.77_{-0.91}^{+1.69}$ & -0.10 & 709.0 & 2.69 & 0.14 & 2.57 & 1.11 & \\
\hline 36 & $12 \quad 35 \quad 55.14$ & +621800.1 & 0.9 & $19.4 \pm 9.9^{\mathrm{a}}$ & $23.5 \pm 8.1$ & $<15.6$ & $<14.2$ & $<0.68$ & $1.40^{\mathrm{d}}$ & 492.2 & 0.40 & 0.21 & $<0.67$ & $<1.01$ & \\
\hline 37 & $1235 \quad 55.33$ & +621504.8 & 0.7 & $37.8 \pm 10.9$ & $36.2 \pm 8.8$ & $<17.8$ & $<12.9$ & $<0.50$ & $>1.46$ & 823.9 & 0.45 & 0.20 & $<0.45$ & $<0.55$ & \\
\hline 38 & 123556.02 & +621555.9 & 0.8 & $1066.6 \pm 36.3$ & $720.5 \pm 29.4$ & $360.9 \pm 23.0$ & $109.3 \pm 15.1$ & $0.51_{-0.04}^{+0.04}$ & 1.45 & 784.7 & 13.54 & 4.09 & 9.67 & 4.90 & \\
\hline 39 & $1235 \quad 56.12$ & $+6212 \quad 19.1$ & 0.7 & $398.8 \pm 23.4$ & $42.8 \pm 9.1$ & $363.9 \pm 22.2$ & $142.7 \pm 14.9$ & $8.60_{-1.59}^{+2.36}$ & -1.00 & 866.4 & 15.19 & 0.22 & 15.44 & 7.92 & \\
\hline
\end{tabular}


Table 3-Continued

\begin{tabular}{|c|c|c|c|c|c|c|c|c|c|c|c|c|c|c|c|c|}
\hline \multirow[b]{2}{*}{$\begin{array}{c}\# \\
(1)\end{array}$} & \multicolumn{2}{|c|}{ Coordinates } & \multirow{2}{*}{$\begin{array}{l}\text { Positional } \\
\text { Error } \\
(4)\end{array}$} & \multicolumn{4}{|c|}{ Counts } & \multirow{2}{*}{$\begin{array}{l}\text { Band } \\
\text { Ratio } \\
(9)\end{array}$} & \multirow{2}{*}{$\begin{array}{c}\text { Effective } \\
\Gamma \\
(10)\end{array}$} & \multirow{2}{*}{$\begin{array}{c}\text { Exposure } \\
\text { Exposure }(\mathrm{ks}) \\
(11)\end{array}$} & \multirow[b]{2}{*}{$\begin{array}{l}\text { FB } \\
(12)\end{array}$} & \multicolumn{3}{|c|}{ Flux } & \multirow{2}{*}{\multicolumn{2}{|c|}{$\begin{array}{l}\text { Notes } \\
(16)\end{array}$}} \\
\hline & $\begin{array}{c}\alpha_{2000} \\
(2)\end{array}$ & $\begin{array}{c}\delta_{2000} \\
(3)\end{array}$ & & $\begin{array}{l}\text { FB } \\
(5)\end{array}$ & $\begin{array}{l}\text { SB } \\
(6)\end{array}$ & $\begin{array}{l}\mathrm{HB} \\
(7)\end{array}$ & $\begin{array}{l}\text { UHB } \\
(8)\end{array}$ & & & & & $\begin{array}{l}\text { SB } \\
(13)\end{array}$ & $\begin{array}{c}\mathrm{HB} \\
(14)\end{array}$ & $\begin{array}{l}\text { UHB } \\
(15)\end{array}$ & & \\
\hline 40 & 123556.30 & +621617.2 & 0.8 & $93.2 \pm 13.7$ & $87.7 \pm 11.9$ & $<17.9$ & $<14.5$ & $<0.21$ & $>2.00$ & 747.6 & 0.91 & 0.54 & $<0.45$ & $<0.64$ & & \\
\hline 41 & $1235 \quad 57.46$ & +621516.6 & 0.7 & $75.7 \pm 12.6$ & $42.5 \pm 9.3$ & $35.3 \pm 9.7$ & $<16.9$ & $0.84_{-0.28}^{+0.33}$ & 0.98 & 851.2 & 1.15 & 0.22 & 0.96 & $<0.74$ & & \\
\hline 42 & $1235 \quad 57.68$ & +621024.6 & 0.8 & $71.2 \pm 12.3$ & $50.5 \pm 9.5$ & $<20.3$ & $<16.0$ & $<0.41$ & $>1.61$ & 524.5 & 1.23 & 0.43 & $<0.79$ & $<1.07$ & & \\
\hline 43 & $1235 \quad 57.90$ & $+62 \quad 13 \quad 10.2$ & 0.7 & $58.7 \pm 11.6$ & $52.3 \pm 9.5$ & $<18.2$ & $<14.4$ & $<0.35$ & $>1.72$ & 869.1 & 0.58 & 0.27 & $<0.42$ & $<0.56$ & & \\
\hline 44 & $1235 \quad 57.98$ & $\begin{array}{l}+622112.3 \\
\end{array}$ & 1.1 & $38.9 \pm 12.2$ & $21.8 \pm 8.2$ & $<23.6$ & $<18.3$ & $<1.10$ & $1.40^{\mathrm{d}}$ & 427.8 & 0.93 & 0.23 & $<1.17$ & $<1.54$ & & \\
\hline 45 & $1235 \quad 58.53$ & +621101.8 & 0.8 & $28.8 \pm 10.3$ & $15.5 \pm 7.1$ & $<20.7$ & $<17.7$ & $<1.37$ & $1.40^{\mathrm{d}}$ & 783.6 & 0.38 & 0.09 & $<0.56$ & $<0.81$ & & \\
\hline 46 & $1235 \quad 58.92$ & +621543.0 & 0.7 & $<24.5$ & $13.1 \pm 7.3^{\mathrm{a}}$ & $<17.4$ & $<16.3$ & $<1.36$ & $1.40^{\mathrm{d}}$ & 823.4 & $<0.31$ & 0.07 & $<0.45$ & $<0.70$ & & \\
\hline 47 & $1235 \quad 59.71$ & +621549.9 & 0.7 & $29.3 \pm 10.1$ & $30.5 \pm 8.5$ & $<16.4$ & $<15.9$ & $<0.54$ & $>1.39$ & 835.8 & 0.36 & 0.16 & $<0.42$ & $<0.67$ & & \\
\hline 48 & 123600.10 & +621816.3 & 0.8 & $45.0 \pm 10.7$ & $15.7 \pm 6.7^{\mathrm{b}}$ & $30.2 \pm 9.1$ & $<15.8$ & $1.95_{-0.83}^{+1.59}$ & 0.16 & 502.9 & 1.77 & 0.14 & 1.69 & $<1.28$ & & \\
\hline 49 & 123600.45 & +621925.5 & 0.9 & $54.0 \pm 11.7$ & $30.3 \pm 8.3$ & $23.3 \pm 9.0$ & $<17.0$ & $0.78_{-0.35}^{+0.45}$ & 1.05 & 476.6 & 1.41 & 0.28 & 1.12 & $<1.32$ & & \\
\hline 50 & 123601.55 & +621406.0 & 0.7 & $65.2 \pm 11.6$ & $43.5 \pm 8.8$ & $18.7 \pm 8.1$ & $<14.1$ & $0.43_{-0.20}^{+0.22}$ & 1.57 & 856.3 & 0.71 & 0.23 & 0.45 & $<0.56$ & & \\
\hline 51 & 123603.08 & +622330.5 & 1.3 & $106.7 \pm 16.2$ & $46.2 \pm 10.4$ & $60.2 \pm 13.1$ & $<25.9$ & $1.33_{-0.38}^{+0.48}$ & 0.52 & 276.4 & 6.40 & 0.72 & 5.63 & $<3.81$ & & \\
\hline 52 & $12 \quad 3603.33$ & $\begin{array}{lll}+6211 & 10.7\end{array}$ & 0.7 & $22.7 \pm 9.1$ & $22.7 \pm 7.4$ & $<16.0$ & $<13.1$ & $<0.72$ & $1.40^{\mathrm{d}}$ & 798.4 & 0.29 & 0.13 & $<0.43$ & $<0.59$ & & I \\
\hline 53 & 123604.89 & $+62 \quad 2014.5$ & 1.0 & $180.3 \pm 19.6$ & $181.0 \pm 17.4$ & $<29.9$ & $<20.4$ & $<0.17$ & $>2.08$ & 375.3 & 3.35 & 2.22 & $<1.48$ & $<1.77$ & $\mathrm{~S}$ & co \\
\hline 54 & 123605.40 & +622010.8 & 1.0 & $157.6 \pm 18.1$ & $103.9 \pm 13.3$ & $47.7 \pm 17.8$ & $13.2 \pm 16.8$ & $0.47_{-0.18}^{+0.19}$ & 1.52 & 375.5 & 4.02 & 1.24 & 2.63 & 1.23 & $\mathrm{~S}$ & 1 \\
\hline 55 & $12 \quad 3605.74$ & +621004.0 & 0.7 & $84.7 \pm 13.0$ & $32.7 \pm 8.3$ & $55.7 \pm 11.1$ & $26.6 \pm 8.7^{\mathrm{b}}$ & $1.73_{-0.49}^{+0.18}$ & 0.26 & 837.2 & 1.90 & 0.17 & 1.83 & 1.31 & & \\
\hline 56 & 123605.77 & +621147.8 & 0.6 & $127.0 \pm 14.3$ & $85.5 \pm 11.3$ & $39.3 \pm 9.4$ & $<15.0$ & $0.47_{-013}^{+0.13}$ & 1.51 & 814.3 & 1.50 & 0.47 & 1.00 & $<0.64$ & & \\
\hline 57 & 123605.85 & +620837.9 & 0.9 & $100.1 \pm 12.8$ & $65.1 \pm 9.9$ & $39.6 \pm 9.1$ & $<15.5$ & $0.61_{-0.16}^{+0.18}$ & 1.28 & 317.1 & 3.45 & 0.91 & 2.71 & $<1.78$ & & \\
\hline 58 & 123606.43 & +621232.2 & 0.6 & $43.7 \pm 9.8$ & $19.8 \pm 6.6$ & $23.8 \pm 8.0$ & $16.6 \pm 7.0$ & $1.22_{-0.74}^{+0.74}$ & 0.60 & 875.4 & 0.79 & 0.10 & 0.69 & 0.73 & & \\
\hline 59 & 123606.60 & +620951.2 & 0.7 & $92.8 \pm 13.3$ & $10.4 \pm 6.4^{\mathrm{b}}$ & $80.3 \pm 12.2$ & $52.7 \pm 10.3$ & $7.82_{-3.21}^{+12.60}$ & -0.96 & 826.3 & 3.64 & 0.06 & 3.55 & 3.10 & & \\
\hline 60 & 123606.72 & +621550.7 & 0.6 & $177.8 \pm 16.3$ & $141.9 \pm 13.8$ & $37.2 \pm 9.2$ & $<16.1$ & $0.27_{-0.07}^{+0.07}$ & 1.88 & 883.9 & 1.57 & 0.73 & 0.81 & $<0.60$ & & \\
\hline 61 & 123606.77 & +621220.9 & 0.6 & $27.4 \pm 8.8$ & $24.8 \pm 7.2$ & $<13.7$ & $<10.2$ & $<0.56$ & $1.40^{\mathrm{d}}$ & 860.2 & 0.33 & 0.13 & $<0.34$ & $<0.42$ & & \\
\hline 62 & 123607.68 & $\begin{array}{r}620850.6 \\
\end{array}$ & 0.8 & $<16.3$ & $7.1 \pm 5.7^{\mathrm{a}}$ & $<10.3$ & $<10.2$ & $<1.48$ & $1.40^{\mathrm{d}}$ & 326.1 & $<0.51$ & 0.10 & $<0.67$ & $<1.12$ & & \\
\hline 63 & $12 \quad 36 \quad 07.71$ & +621503.4 & 0.6 & $416.9 \pm 23.2$ & $289.0 \pm 18.8$ & $123.1 \pm 13.7$ & $42.3 \pm 9.0$ & $0.43_{-0.05}^{+0.06}$ & 1.58 & 855.6 & 4.51 & 1.51 & 2.93 & 1.69 & & \\
\hline 64 & 123608.15 & +621036.0 & 0.7 & $59.0 \pm 11.4$ & $23.4 \pm 7.5$ & $35.3 \pm 9.6$ & $<17.6$ & $1.54_{-0.56}^{+0.83}$ & 0.37 & 826.9 & 1.27 & 0.12 & 1.14 & $<0.86$ & & \\
\hline 65 & 123608.25 & $+6215 \quad 53.1$ & 0.6 & $113.3 \pm 13.6$ & $23.0 \pm 7.1$ & $88.4 \pm 12.1$ & $39.0 \pm 8.9$ & $3.89_{-1.06}^{+1.56}$ & -0.34 & 879.7 & 3.22 & 0.11 & 3.18 & 1.95 & & \\
\hline 66 & 123608.61 & +621435.9 & 0.6 & $24.8 \pm 8.2^{\mathrm{b}}$ & $19.2 \pm 6.5$ & $<12.8$ & $<12.0$ & $<0.68$ & $1.40^{\mathrm{d}}$ & 833.4 & 0.30 & 0.10 & $<0.32$ & $<0.50$ & & \\
\hline 67 & 123608.95 & +621821.9 & 0.8 & $<19.1$ & $12.6 \pm 6.1^{\mathrm{a}}$ & $<13.4$ & $<11.3$ & $<1.07$ & $1.40^{\mathrm{d}}$ & 471.0 & $<0.42$ & 0.12 & $<0.60$ & $<0.83$ & & \\
\hline 68 & 123609.77 & +621146.5 & 0.6 & $23.4 \pm 8.3^{\mathrm{b}}$ & $14.5 \pm 6.2^{\mathrm{a}}$ & $<15.2$ & $<11.8$ & $<1.06$ & $1.40^{\mathrm{d}}$ & 764.6 & 0.31 & 0.08 & $<0.42$ & $<0.55$ & & \\
\hline 69 & 123611.10 & +622228.4 & 1.1 & $1046.4 \pm 38.6$ & $396.9 \pm 24.5$ & $631.4 \pm 30.9$ & $193.5 \pm 20.4$ & $1.60_{-0.12}^{+0.13}$ & 0.33 & 458.5 & 41.48 & 3.77 & 36.89 & 17.20 & & \\
\hline 70 & $1236 \quad 11.29$ & +620903.9 & 0.8 & $141.4 \pm 21.9$ & $<21.2$ & $111.8 \pm 21.4$ & $40.1 \pm 18.1$ & $>5.34$ & $<-0.67$ & 564.6 & 7.23 & $<0.17$ & 6.76 & 3.32 & $\mathrm{~S}$ & \\
\hline 71 & 123611.43 & +622152.6 & 1.1 & $190.4 \pm 18.6$ & $34.2 \pm 9.5$ & $155.1 \pm 16.8^{\mathrm{c}}$ & $77.2 \pm 12.8^{\mathrm{c}}$ & $4.55_{-1.10}^{+1.81}$ & -0.49 & 471.1 & 10.79 & 0.32 & 10.67 & 7.37 & $\mathrm{O}$ & \\
\hline 72 & 123611.84 & $\begin{array}{r}621014.7\end{array}$ & 0.7 & $75.0 \pm 12.2$ & $<15.1$ & $67.9 \pm 11.4$ & $31.8 \pm 8.8$ & $>4.54$ & $<-0.49$ & 876.1 & 2.28 & $<0.08$ & 2.53 & 1.63 & & \\
\hline 73 & 123611.97 & $\begin{array}{r}+620859.9\end{array}$ & 0.8 & $100.5 \pm 17.8$ & $50.7 \pm 13.1$ & $<22.0$ & $<16.4$ & $<0.44$ & $>1.56$ & 594.5 & 1.58 & 0.38 & $<0.76$ & $<0.96$ & $\mathrm{~S}$ & \\
\hline 74 & $1236 \quad 12.02$ & +621138.9 & 0.6 & $1513.8 \pm 40.8$ & $1011.2 \pm 33.3$ & $506.8 \pm 24.7$ & $166.6 \pm 15.5$ & $0.51_{-0.03}^{+0.03}$ & 1.45 & 762.9 & 19.78 & 5.90 & 13.96 & 7.69 & & \\
\hline 75 & 123612.07 & +621940.8 & 0.8 & $991.0 \pm 34.3$ & $749.9 \pm 29.3$ & $251.5 \pm 19.4$ & $88.6 \pm 13.2$ & $0.34_{-0.03}^{+0.03}$ & 1.74 & 380.5 & 21.96 & 8.89 & 13.02 & 7.79 & & \\
\hline 76 & $12 \quad 36 \quad 12.93$ & +621929.8 & 0.8 & $36.6 \pm 9.9$ & $31.5 \pm 8.0$ & $<14.5$ & $<12.8$ & $<0.47$ & $>1.52$ & 380.8 & 0.92 & 0.37 & $<0.79$ & $<1.15$ & & \\
\hline 77 & $1236 \quad 13.07$ & $\begin{array}{r}621224.1\end{array}$ & 0.6 & $74.2 \pm 11.1$ & $23.0 \pm 6.7$ & $53.9 \pm 9.8$ & $26.0 \pm 7.4$ & $2.39^{+1.08}$ & 0.01 & 775.3 & 2.04 & 0.13 & 2.03 & 1.42 & & \\
\hline
\end{tabular}


Table 3-Continued

\begin{tabular}{|c|c|c|c|c|c|c|c|c|c|c|c|c|c|c|c|c|}
\hline \multirow[b]{2}{*}{$\begin{array}{c}\# \\
(1)\end{array}$} & \multicolumn{2}{|c|}{ Coordinates } & \multirow{2}{*}{$\begin{array}{c}\text { Positional } \\
\text { Error } \\
(4)\end{array}$} & \multicolumn{4}{|c|}{ Counts } & \multirow{2}{*}{$\begin{array}{c}\text { Band } \\
\text { Ratio } \\
(9)\end{array}$} & \multirow{2}{*}{$\begin{array}{c}\text { Effective } \\
\Gamma \\
(10)\end{array}$} & \multirow{2}{*}{$\begin{array}{c}\text { Exposure } \\
\text { Exposure (ks) } \\
\text { (11) }\end{array}$} & \multirow[b]{2}{*}{$\begin{array}{l}\text { FB } \\
(12)\end{array}$} & \multicolumn{3}{|c|}{ Flux } & \multirow{2}{*}{\multicolumn{2}{|c|}{$\begin{array}{l}\text { Notes } \\
(16)\end{array}$}} \\
\hline & $\begin{array}{c}\alpha_{2000} \\
(2)\end{array}$ & $\begin{array}{c}\delta_{2000} \\
(3)\end{array}$ & & $\begin{array}{l}\text { FB } \\
(5)\end{array}$ & $\begin{array}{l}\text { SB } \\
(6)\end{array}$ & $\begin{array}{l}\mathrm{HB} \\
(7)\end{array}$ & $\begin{array}{l}\text { UHB } \\
(8)\end{array}$ & & & & & $\begin{array}{l}\text { SB } \\
(13)\end{array}$ & $\begin{array}{l}\text { HB } \\
(14)\end{array}$ & $\begin{array}{l}\text { UHB } \\
(15)\end{array}$ & & \\
\hline 78 & $12 \quad 36 \quad 14.11$ & +621017.4 & 0.6 & $25.4 \pm 9.2$ & $7.3 \pm 5.8^{\mathrm{b}}$ & $15.2 \pm 7.9^{\mathrm{b}}$ & $<15.6$ & $2.12_{-1.44}^{+8.41}$ & 0.10 & 880.7 & 0.59 & 0.04 & 0.49 & $<0.73$ & & \\
\hline 79 & 123614.45 & +621045.6 & 0.6 & $84.2 \pm 12.2$ & $18.7 \pm 6.7$ & $66.7 \pm 11.0$ & $23.8 \pm 7.8$ & $3.59_{-1.12}^{+2.10}$ & -0.28 & 890.0 & 2.30 & 0.09 & 2.32 & 1.16 & C & \\
\hline 80 & $12 \quad 36 \quad 14.47$ & +621318.8 & 0.6 & $33.4 \pm 8.3$ & $17.6 \pm 6.1$ & $17.0 \pm 6.8^{\mathrm{b}}$ & $<12.8$ & $0.98_{-0.47}^{+0.125}$ & 0.83 & 864.3 & 0.54 & 0.09 & 0.47 & $<0.56$ & C & \\
\hline 81 & 123614.56 & $\begin{array}{llll}+62 & 07 & 17.7\end{array}$ & 0.9 & $17.4 \pm 7.9^{\mathrm{b}}$ & $14.6 \pm 6.2^{\mathrm{a}}$ & $<12.3$ & $<9.6$ & $<0.85$ & $1.40^{\mathrm{d}}$ & 221.3 & 0.81 & 0.29 & $<1.18$ & $<1.56$ & & \\
\hline 82 & 123614.95 & +621026.8 & 0.6 & $23.0 \pm 8.8$ & $14.6 \pm 6.3^{\mathrm{a}}$ & $<16.1$ & $<14.6$ & $<1.11$ & $1.40^{\mathrm{d}}$ & 883.8 & 0.27 & 0.07 & $<0.38$ & $<0.58$ & $\mathrm{C}$ & \\
\hline 83 & 123615.89 & +621515.5 & 0.6 & $81.5 \pm 18.7$ & $<20.7$ & $71.9 \pm 20.7$ & $36.7 \pm 17.1$ & $>3.52$ & $<-0.26$ & 896.4 & 2.19 & $<0.10$ & 2.48 & 1.76 & $\mathrm{C}, \mathrm{S}$ & \\
\hline 84 & 123616.03 & +621107.9 & 0.6 & $656.8 \pm 28.6$ & $218.2 \pm 16.5$ & $439.6 \pm 23.7$ & $165.4 \pm 15.2$ & $2.03_{-0.18}^{+0.20}$ & 0.13 & 884.3 & 14.94 & 1.07 & 13.99 & 7.69 & $\mathrm{C}$ & \\
\hline 85 & 123616.11 & +621513.5 & 0.6 & $95.6 \pm 21.2$ & $25.9 \pm 17.2$ & $43.4 \pm 14.0$ & $21.6 \pm 11.1$ & $1.70_{-0.87}^{+3.40}$ & 0.28 & 896.8 & 1.99 & 0.13 & 1.32 & 0.97 & $\mathrm{C}, \mathrm{S}$ & \\
\hline 86 & 123617.05 & +621011.3 & 0.6 & $415.1 \pm 23.3$ & $278.2 \pm 18.5$ & $130.2 \pm 14.1$ & $44.1 \pm 9.3$ & $0.47_{-0.06}^{+0.06}$ & 1.51 & 879.9 & 4.54 & 1.41 & 3.05 & 1.73 & C & \\
\hline 87 & 123617.64 & +621344.1 & 0.6 & $25.0 \pm 7.4$ & $<11.7$ & $17.4 \pm 6.6^{\mathrm{b}}$ & $10.0 \pm 5.6^{\mathrm{b}}$ & $>1.51$ & $<0.39$ & 851.2 & 0.52 & $<0.06$ & 0.54 & 0.47 & $\mathrm{C}$ & \\
\hline 88 & 123618.02 & +621635.4 & 0.6 & $1381.3 \pm 39.0$ & $935.7 \pm 32.1$ & $461.5 \pm 23.7$ & $139.1 \pm 14.4$ & $0.50_{-0.03}^{+0.03}$ & 1.46 & 904.2 & 15.11 & 4.61 & 10.69 & 5.36 & C & \\
\hline 89 & 123618.58 & +621115.0 & 0.6 & $1723.6 \pm 43.3$ & $1319.9 \pm 37.7$ & $418.1 \pm 22.7$ & $93.5 \pm 12.6$ & $0.32_{-0.02}^{+0.02}$ & 1.78 & 884.8 & 16.08 & 6.75 & 9.21 & 3.52 & C & \\
\hline 90 & 123619.18 & +621441.6 & 0.6 & $335.6 \pm 20.8$ & $239.3 \pm 17.2$ & $93.7 \pm 11.9$ & $28.8 \pm 7.6$ & $0.40_{-0.06}^{+0.06}$ & 1.64 & 906.3 & 3.31 & 1.19 & 2.08 & 1.07 & C & I \\
\hline 91 & $\begin{array}{llll}12 & 36 & 19.17\end{array}$ & +622419.6 & 1.3 & $51.4 \pm 18.7^{\mathrm{bc}}$ & $32.2 \pm 11.8$ & $<39.8$ & $<30.3$ & $<1.30$ & $>0.54$ & 534.9 & 1.57 & 0.26 & $<1.97$ & $<2.42$ & $\mathrm{O}$ & 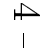 \\
\hline 92 & 123619.55 & +621252.8 & 0.6 & $21.3 \pm 7.1$ & $15.8 \pm 5.8$ & $<10.7$ & $<9.1$ & $<0.69$ & $1.40^{\mathrm{d}}$ & 686.5 & 0.32 & 0.10 & $<0.33$ & $<0.47$ & $\mathrm{C}$ & \\
\hline 93 & 123619.93 & +621911.0 & 0.7 & $36.3 \pm 9.6$ & $18.6 \pm 6.7$ & $19.0 \pm 7.9$ & $<13.5$ & $1.03_{-0.51}^{+0.72}$ & 0.78 & 531.4 & 0.99 & 0.15 & 0.87 & $<0.96$ & & \\
\hline 94 & 123620.52 & +621238.1 & 0.6 & $29.4 \pm 7.9$ & $16.3 \pm 5.8$ & $15.0 \pm 6.3$ & $10.0 \pm 5.4^{\mathrm{b}}$ & $0.93_{-0.65}^{+0.65}$ & 0.88 & 815.0 & 0.49 & 0.09 & 0.44 & 0.46 & $\mathrm{C}$ & \\
\hline 95 & 123620.87 & +621415.2 & 0.6 & $62.8 \pm 12.8$ & $<11.4$ & $54.8 \pm 15.9$ & $36.3 \pm 14.4$ & $>4.88$ & $<-0.57$ & 911.9 & 1.90 & $<0.05$ & 2.00 & 1.79 & $\mathrm{C}, \mathrm{S}$ & \\
\hline 96 & 123621.02 & $+62 \quad 1412.1$ & 0.6 & $203.7 \pm 29.0$ & $109.4 \pm 21.9$ & $63.9 \pm 18.0$ & $42.2 \pm 16.0$ & $0.59_{-0.19}^{+0.22}$ & 1.32 & 910.1 & 2.40 & 0.53 & 1.51 & 1.63 & $\mathrm{C}, \mathrm{S}$ & \\
\hline 97 & 123621.11 & +621303.6 & 0.6 & $19.2 \pm 6.8^{\mathrm{a}}$ & $9.1 \pm 4.8^{\mathrm{b}}$ & $<11.9$ & $<10.0$ & $<1.33$ & $1.40^{\mathrm{d}}$ & 664.7 & 0.30 & 0.06 & $<0.38$ & $<0.53$ & $\mathrm{C}$ & \\
\hline 98 & 123621.22 & +621108.8 & 0.6 & $98.7 \pm 12.4$ & $17.0 \pm 6.1^{\mathrm{b}}$ & $82.0 \pm 11.4$ & $38.9 \pm 8.6$ & $4.84_{-1.44}^{+2.79}$ & -0.56 & 892.0 & 3.04 & 0.08 & 3.03 & 1.95 & $\mathrm{C}, \mathrm{NP}$ & \\
\hline 99 & 123621.93 & +621603.2 & 0.6 & $34.8 \pm 8.6$ & $<12.7$ & $27.0 \pm 7.7$ & $20.4 \pm 6.8$ & $>2.15$ & $<0.09$ & 912.2 & 0.78 & $<0.06$ & 0.84 & 0.92 & $\mathrm{C}$ & \\
\hline 100 & 123622.66 & +621028.5 & 0.6 & $418.1 \pm 23.1$ & $289.0 \pm 18.7$ & $120.9 \pm 13.4$ & $35.8 \pm 8.4$ & $0.42_{-0.05}^{+0.06}$ & 1.59 & 874.0 & 4.39 & 1.48 & 2.80 & 1.39 & C & \\
\hline 101 & $12 \quad 3622.95$ & +621526.8 & 0.6 & $1702.7 \pm 42.8$ & $1248.2 \pm 36.6$ & $478.0 \pm 23.6$ & $168.4 \pm 14.9$ & $0.39_{-0.02}^{+0.02}$ & 1.65 & 924.2 & 16.33 & 6.07 & 10.37 & 6.13 & $\mathrm{C}$ & \\
\hline 102 & 123623.06 & +621346.5 & 0.6 & $17.8 \pm 6.4^{\mathrm{a}}$ & $11.7 \pm 5.2^{\mathrm{a}}$ & $<11.5$ & $<9.8$ & $<1.00$ & $1.40^{\mathrm{d}}$ & 838.6 & 0.22 & 0.06 & $<0.29$ & $<0.41$ & $\mathrm{C}$ & \\
\hline 103 & 123623.66 & +621837.6 & 0.7 & $137.2 \pm 14.7$ & $101.2 \pm 12.0$ & $40.4 \pm 9.4$ & $<15.3$ & $0.41_{-0.11}^{+0.11}$ & 1.61 & 697.1 & 1.79 & 0.65 & 1.19 & $<0.77$ & & \\
\hline 104 & 123623.69 & +621009.0 & 0.6 & $33.8 \pm 9.0$ & $17.9 \pm 6.5$ & $14.9 \pm 7.0^{\mathrm{b}}$ & $<10.6$ & $0.84_{-0.45}^{+0.62}$ & 0.99 & 896.1 & 0.49 & 0.09 & 0.38 & $<0.43$ & $\mathrm{C}$ & \\
\hline 105 & 123624.56 & +621111.4 & 0.6 & $91.1 \pm 11.9$ & $57.6 \pm 9.3$ & $29.0 \pm 7.9$ & $15.3 \pm 6.4^{\mathrm{b}}$ & $0.51_{-0.15}^{+0.45}$ & 1.45 & 905.5 & 1.00 & 0.28 & 0.67 & 0.58 & C & \\
\hline 106 & 123625.05 & $+62 \quad 21 \quad 16.4$ & 0.9 & $109.3 \pm 14.6$ & $94.9 \pm 12.3$ & $<19.1$ & $<14.6$ & $<0.20$ & $>2.01$ & 476.5 & 1.66 & 0.91 & $<0.74$ & $<0.99$ & & \\
\hline 107 & 123625.36 & $+62 \quad 1737.7$ & 0.6 & $228.4 \pm 17.7$ & $147.1 \pm 13.9$ & $78.9 \pm 11.3$ & $23.3 \pm 7.3^{\mathrm{b}}$ & $0.55_{-0.09}^{+0.10}$ & 1.38 & 868.2 & 2.72 & 0.75 & 1.94 & 0.96 & & \\
\hline 108 & 123625.41 & +621404.8 & 0.6 & $212.6 \pm 16.8$ & $202.5 \pm 15.8$ & $11.1 \pm 5.6^{\mathrm{b}}$ & $<7.0$ & $0.06_{-0.03}^{+0.03}$ & 2.25 & 839.9 & 1.62 & 1.12 & 0.24 & $<0.26$ & $\mathrm{C}$ & \\
\hline 109 & $1236 \quad 27.28$ & +621308.3 & 0.6 & $10.0 \pm 5.6^{\mathrm{a}}$ & $<10.3$ & $6.0 \pm 4.9^{\mathrm{a}}$ & $<9.7$ & $>0.59$ & $1.40^{\mathrm{d}}$ & 901.0 & 0.11 & $<0.05$ & 0.14 & $<0.38$ & $\mathrm{C}, \mathrm{NP}$ & \\
\hline 110 & $1236 \quad 27.32$ & $+62 \quad 1258.1$ & 0.6 & $<12.2$ & $10.7 \pm 4.9$ & $<5.1$ & $<5.2$ & $<0.48$ & $1.40^{\mathrm{d}}$ & 869.7 & $<0.14$ & 0.05 & $<0.12$ & $<0.21$ & C & \\
\hline 111 & $12 \quad 36 \quad 27.48$ & +621026.0 & 0.6 & $133.9 \pm 14.0$ & $32.3 \pm 7.5$ & $107.2 \pm 12.7$ & $43.9 \pm 8.9$ & $3.34_{-0.74}^{+1.08}$ & -0.22 & 883.7 & 3.59 & 0.16 & 3.70 & 2.12 & $\mathrm{C}$ & \\
\hline 112 & 123627.54 & +621218.2 & 0.6 & $28.3 \pm 7.4$ & $20.4 \pm 6.1$ & $9.9 \pm 5.6^{\mathrm{b}}$ & $<10.3$ & $0.49_{-0.30}^{+0.34}$ & 1.48 & 905.1 & 0.31 & 0.10 & 0.23 & $<0.39$ & $\mathrm{C}$ & \\
\hline 113 & 123627.76 & +621158.4 & 0.6 & $85.7 \pm 11.3$ & $22.1 \pm 6.3$ & $68.0 \pm 10.3$ & $37.2 \pm 8.1$ & $3.09_{-0.33}^{+1.32}$ & -0.17 & 919.4 & 2.16 & 0.10 & 2.23 & 1.71 & $\mathrm{C}$ & \\
\hline 114 & 123628.60 & +622139.1 & 1.0 & $853.1 \pm 33.0$ & $494.4 \pm 24.5$ & $342.4 \pm 21.8$ & $104.0 \pm 13.2^{\mathrm{c}}$ & $0.69_{-0.05}^{+0.06}$ & 1.17 & 471.0 & 21.08 & 4.63 & 15.99 & 7.91 & $\mathrm{O}$ & \\
\hline 115 & $12 \quad 36 \quad 28.77$ & +620806.4 & 0.7 & $44.8 \pm 10.9$ & $22.5 \pm 7.6$ & $19.1 \pm 8.6^{\mathrm{b}}$ & $14.3 \pm 7.6^{\mathrm{b}}$ & $0.85_{-0.44}^{+0.58}$ & 0.97 & 755.1 & 0.77 & 0.13 & 0.59 & 0.70 & & \\
\hline
\end{tabular}


Table 3-Continued

\begin{tabular}{|c|c|c|c|c|c|c|c|c|c|c|c|c|c|c|c|}
\hline \multirow[b]{2}{*}{$\begin{array}{l}\# \\
(1)\end{array}$} & \multicolumn{2}{|c|}{ Coordinates } & \multirow{2}{*}{$\begin{array}{l}\text { Positional } \\
\text { Error } \\
(4)\end{array}$} & \multicolumn{4}{|c|}{ Counts } & \multirow{2}{*}{$\begin{array}{l}\text { Band } \\
\text { Ratio } \\
(9)\end{array}$} & \multirow{2}{*}{$\begin{array}{c}\text { Effective } \\
\Gamma \\
(10)\end{array}$} & \multirow{2}{*}{$\begin{array}{c}\text { Exposure } \\
\text { Exposure }(\mathrm{ks}) \\
(11)\end{array}$} & \multirow[b]{2}{*}{$\begin{array}{l}\text { FB } \\
(12)\end{array}$} & \multicolumn{3}{|c|}{ Flux } & \multirow[b]{2}{*}{$\begin{array}{l}\text { Notes } \\
(16)\end{array}$} \\
\hline & $\begin{array}{c}\alpha_{2000} \\
(2)\end{array}$ & $\begin{array}{c}\delta_{2000} \\
(3)\end{array}$ & & $\begin{array}{l}\text { FB } \\
(5)\end{array}$ & $\begin{array}{l}\text { SB } \\
(6)\end{array}$ & $\begin{array}{l}\text { HB } \\
(7)\end{array}$ & $\begin{array}{l}\text { UHB } \\
(8)\end{array}$ & & & & & $\begin{array}{l}\text { SB } \\
(13)\end{array}$ & $\begin{array}{c}\mathrm{HB} \\
(14)\end{array}$ & $\begin{array}{l}\text { UHB } \\
(15)\end{array}$ & \\
\hline 116 & 123628.89 & +6206 16.1 & 0.9 & $128.1 \pm 14.8$ & $40.7 \pm 8.8$ & $91.0 \pm 12.8$ & $29.7 \pm 8.6$ & $2.26_{-0.51}^{+0.70}$ & 0.04 & 346.4 & 7.74 & 0.51 & 7.57 & 3.64 & \\
\hline 117 & 123629.11 & +621045.9 & 0.6 & $102.7 \pm 12.4$ & $33.9 \pm 7.6$ & $71.1 \pm 10.7$ & $24.4 \pm 7.2$ & $2.11_{-0.50}^{+0.69}$ & 0.10 & 907.9 & 2.31 & 0.16 & 2.22 & 1.10 & $\mathrm{C}$ \\
\hline 118 & 123629.34 & +621613.8 & 0.6 & $74.6 \pm 10.8$ & $13.2 \pm 5.4$ & $64.4 \pm 10.1$ & $33.3 \pm 7.8$ & $4.95_{-1}^{+3.50}$ & -0.58 & 912.0 & 2.27 & 0.06 & 2.36 & 1.64 & $\mathrm{C}$ \\
\hline 119 & 123629.45 & +621513.1 & 0.6 & $178.9 \pm 15.6$ & $138.7 \pm 13.3$ & $42.7 \pm 8.6$ & $13.7 \pm 5.9$ & $0.31_{-0.07}^{+0.03}$ & 1.79 & 923.5 & 1.59 & 0.68 & 0.90 & 0.49 & $\mathrm{C}$ \\
\hline 120 & 123629.52 & +622213.8 & 1.0 & $508.2 \pm 26.6$ & $368.7 \pm 21.6$ & $145.7 \pm 16.1$ & $54.6 \pm 11.3$ & $0.40_{-0.05}^{+0.05}$ & 1.64 & 473.6 & 9.59 & 3.50 & 6.14 & 3.94 & \\
\hline 121 & 123630.06 & +620923.7 & 0.6 & $55.4 \pm 10.6$ & $12.6 \pm 6.0^{\mathrm{b}}$ & $46.6 \pm 9.7$ & $<15.8$ & $3.70_{-1.42}^{+3.44}$ & -0.30 & 891.1 & 1.53 & 0.06 & 1.62 & $<0.77$ & $\mathrm{C}$ \\
\hline 122 & 123630.05 & +620542.8 & 1.0 & $68.0 \pm 12.1$ & $<14.1$ & $63.2 \pm 11.3$ & $52.3 \pm 10.2$ & $>4.49$ & $<-0.48$ & 320.7 & 5.63 & $<0.20$ & 6.43 & 7.48 & NP \\
\hline 123 & 123630.21 & +622620.8 & 1.5 & $844.1 \pm 37.3$ & $537.2 \pm 27.3$ & $290.9 \pm 25.1$ & $<48.3$ & $0.58_{-0.06}^{+0.06}$ & 1.33 & 523.7 & 17.12 & 4.52 & 12.53 & $<3.75$ & \\
\hline 124 & 123631.27 & +620958.3 & 0.6 & $18.6 \pm 7.4$ & $17.8 \pm 6.3$ & $<11.0$ & $<7.2$ & $<0.62$ & $1.40^{\mathrm{d}}$ & 879.0 & 0.22 & 0.09 & $<0.26$ & $<0.29$ & $\mathrm{C}$ \\
\hline 125 & 123631.68 & +620907.8 & 0.6 & $20.7 \pm 8.4^{\mathrm{a}}$ & $17.5 \pm 6.5^{\mathrm{a}}$ & $<15.1$ & $<11.7$ & $<0.87$ & $1.40^{\mathrm{d}}$ & 840.5 & 0.25 & 0.09 & $<0.38$ & $<0.48$ & $\mathrm{C}$ \\
\hline 126 & 123631.92 & +621744.2 & 0.6 & $91.5 \pm 21.2$ & $26.8 \pm 12.5$ & $66.4 \pm 18.5$ & $<12.1$ & $2.54_{-1.07}^{+2.33}$ & -0.04 & 766.2 & 2.60 & 0.15 & 2.57 & $<0.68$ & M \\
\hline 127 & 123632.43 & +620759.7 & 0.7 & $63.7 \pm 12.0$ & $29.0 \pm 8.1$ & $40.7 \pm 10.2$ & $25.0 \pm 8.6$ & $1.41_{-0.65}^{+0.05}$ & 0.46 & 852.5 & 1.27 & 0.15 & 1.24 & 1.15 & \\
\hline 128 & 123632.77 & +622028.1 & 0.8 & $171.7 \pm 16.8$ & $173.7 \pm 15.3$ & $<18.1$ & $<14.1$ & $<0.10$ & $>2.22$ & 674.2 & 1.65 & 1.19 & $<0.48$ & $<0.66$ & \\
\hline 129 & 123633.21 & +620834.3 & 0.7 & $641.4 \pm 28.6$ & $508.5 \pm 24.5$ & $130.0 \pm 14.3$ & $29.4 \pm 8.4$ & $0.26_{-0.03}^{+0.03}$ & 1.90 & 801.8 & 6.17 & 2.89 & 3.07 & 1.20 & \\
\hline 130 & 123633.49 & +621418.1 & 0.6 & $110.7 \pm 12.4$ & $86.6 \pm 10.7$ & $28.8 \pm 7.2$ & $<10.6$ & $0.34_{-0.09}^{+0.10}$ & 1.74 & 929.8 & 1.00 & 0.42 & 0.61 & $<0.38$ & C \\
\hline 131 & 123633.68 & +621005.7 & 0.6 & $66.3 \pm 10.6$ & $40.4 \pm 8.1$ & $28.1 \pm 7.9$ & $16.1 \pm 6.6^{\mathrm{b}}$ & $0.70_{-0.23}^{+0.26}$ & 1.17 & 908.4 & 0.85 & 0.20 & 0.68 & 0.63 & $\mathrm{C}$ \\
\hline 132 & 123633.76 & +621313.8 & 0.6 & $40.3 \pm 8.3$ & $<9.8$ & $35.6 \pm 7.9$ & $16.6 \pm 6.0$ & $>3.67$ & $<-0.29$ & 932.2 & 1.06 & $<0.05$ & 1.19 & 0.77 & $\mathrm{C}$ \\
\hline 133 & 123633.86 & $+62 \quad 1327.7$ & 0.6 & $<9.7$ & $5.8 \pm 4.2^{\mathrm{a}}$ & $<4.8$ & $<4.8$ & $<0.84$ & $1.40^{\mathrm{d}}$ & 932.2 & $<0.11$ & 0.03 & $<0.11$ & $<0.18$ & $\mathrm{C}$ \\
\hline 134 & 123634.46 & $\begin{array}{r}+621212.9\end{array}$ & 0.6 & $46.4 \pm 8.9$ & $43.2 \pm 8.1$ & $<11.9$ & $<8.4$ & $<0.28$ & $>1.86$ & 850.2 & 0.43 & 0.23 & $<0.27$ & $<0.32$ & $\mathrm{C}$ \\
\hline 135 & 123634.47 & +620941.1 & 0.6 & $25.8 \pm 8.1$ & $13.3 \pm 5.8$ & $11.1 \pm 6.4^{\mathrm{b}}$ & $<12.2$ & $0.84_{-0.55}^{+0.81}$ & $1.40^{\mathrm{d}}$ & 841.6 & 0.31 & 0.07 & 0.28 & $<0.50$ & $\mathrm{C}$ \\
\hline 136 & 123634.51 & +621241.6 & 0.6 & $28.2 \pm 7.3$ & $21.2 \pm 6.2$ & $<11.9$ & $<9.6$ & $<0.56$ & $1.40^{\mathrm{d}}$ & 932.0 & 0.31 & 0.10 & $<0.27$ & $<0.36$ & $\mathrm{C}$ \\
\hline 137 & 123634.56 & +621926.0 & 0.7 & $357.8 \pm 22.1$ & $41.2 \pm 8.7$ & $324.3 \pm 20.9$ & $135.0 \pm 14.3$ & $7.95_{-1.48}^{+2.20}$ & -0.96 & 840.2 & 13.83 & 0.22 & 14.01 & 7.64 & \\
\hline 138 & 123634.62 & $\begin{array}{r}+620438.8 \\
\end{array}$ & 1.1 & $51.6 \pm 11.0$ & $43.2 \pm 8.7$ & $<16.9$ & $<12.3$ & $\begin{array}{l}-1.48 \\
<0.41\end{array}$ & $>1.62$ & 126.4 & 3.69 & 1.50 & $<2.72$ & $<3.41$ & \\
\hline 139 & 123634.90 & $\begin{array}{r}+621628.2 \\
\end{array}$ & 0.6 & $16.8 \pm 6.4$ & $6.3 \pm 4.3^{\mathrm{b}}$ & $9.2 \pm 5.4^{\mathrm{b}}$ & $<6.7$ & $1.48_{-1.36}^{+3.32}$ & $1.40^{\mathrm{d}}$ & 886.4 & 0.19 & 0.03 & 0.22 & $<0.27$ & $\mathrm{C}$ \\
\hline 140 & 123635.27 & +62 1151.7 & 0.6 & $23.9 \pm 7.0$ & $9.9 \pm 4.8$ & $12.7 \pm 5.8^{\mathrm{b}}$ & $<12.1$ & $1.29_{-0.73}^{+1.37}$ & $1.40^{\mathrm{d}}$ & 843.0 & 0.29 & 0.05 & 0.32 & $<0.50$ & $\mathrm{C}$ \\
\hline 141 & 123635.30 & +621109.9 & 0.6 & $67.9 \pm 10.3$ & $36.4 \pm 7.6$ & $30.2 \pm 7.7$ & $<13.1$ & $0.83_{-0.26}^{+0.30}$ & 0.99 & 919.0 & 0.95 & 0.17 & 0.76 & $<0.52$ & $\mathrm{C}$ \\
\hline 142 & 123635.60 & +621424.4 & 0.6 & $150.0 \pm 14.2$ & $59.2 \pm 9.2$ & $96.8 \pm 11.8$ & $42.0 \pm 8.3$ & $1.66_{-0.30}^{+0.37}$ & 0.30 & 932.9 & 2.97 & 0.28 & 2.82 & 1.82 & $\mathrm{C}$ \\
\hline 143 & 123635.63 & +620708.4 & 0.8 & $141.0 \pm 16.0$ & $<20.3$ & $129.4 \pm 15.0$ & $84.4 \pm 12.4$ & $>6.42$ & $<-0.86$ & 867.0 & 5.08 & $<0.10$ & 5.31 & 4.62 & NP \\
\hline 144 & 123636.64 & +621346.9 & 0.6 & $1171.7 \pm 35.5$ & $913.4 \pm 31.4$ & $266.5 \pm 17.9$ & $59.1 \pm 9.7$ & $0.29_{-0.02}^{+0.02}$ & 1.82 & 933.5 & 10.09 & 4.43 & 5.53 & 2.09 & $\mathrm{C}$ \\
\hline 145 & 123636.75 & +621156.0 & 0.6 & $503.0 \pm 24.9$ & $375.4 \pm 21.0$ & $126.8 \pm 13.3$ & $52.3 \pm 9.2$ & $0.34_{-0.04}^{+0.04}$ & 1.74 & 859.7 & 4.94 & 1.97 & 2.90 & 2.02 & $\mathrm{C}$ \\
\hline 146 & 123636.79 & +622227.5 & 1.0 & $145.6 \pm 16.8$ & $111.9 \pm 13.3$ & $40.3 \pm 11.3$ & $<21.5$ & $0.36_{-0.11}^{+0.11}$ & 1.70 & 478.2 & 2.63 & 1.06 & 1.67 & $<1.54$ & \\
\hline 147 & 123636.89 & +621320.2 & 0.6 & $18.2 \pm 6.3$ & $7.6 \pm 4.4^{\mathrm{b}}$ & $11.9 \pm 5.6^{\mathrm{b}}$ & $<9.7$ & $1.58_{-0.33}^{+2.11}$ & $1.40^{\mathrm{d}}$ & 935.8 & 0.20 & 0.04 & 0.27 & $<0.36$ & $\mathrm{C}$ \\
\hline 148 & 123637.01 & +621134.9 & 0.6 & $<10.3$ & $8.0 \pm 4.5^{\mathrm{a}}$ & $<3.8$ & $<3.9$ & $<0.48$ & $1.40^{\mathrm{d}}$ & 881.6 & $<0.12$ & 0.04 & $<0.09$ & $<0.15$ & $\mathrm{C}$ \\
\hline 149 & 123638.50 & +621339.5 & 0.6 & $24.4 \pm 6.9$ & $<8.9$ & $23.8 \pm 6.8$ & $<11.0$ & $>2.70$ & $<-0.08$ & 937.5 & 0.58 & $<0.04$ & 0.75 & $<0.49$ & $\mathrm{C}$ \\
\hline 150 & 123638.97 & +621041.4 & 0.6 & $51.4 \pm 9.4$ & $33.7 \pm 7.5$ & $17.2 \pm 6.6^{\mathrm{b}}$ & $<9.1$ & $0.51_{-0.22}^{+0.24}$ & 1.44 & 919.8 & 0.56 & 0.16 & 0.39 & $<0.34$ & $\mathrm{C}$ \\
\hline 151 & 123639.07 & +620943.9 & 0.6 & $144.0 \pm 14.4$ & $94.7 \pm 11.4$ & $47.4 \pm 9.3$ & $17.8 \pm 6.7^{\mathrm{b}}$ & $0.50_{-0.11}^{+0.12}$ & 1.46 & 821.1 & 1.74 & 0.51 & 1.20 & 0.74 & $\mathrm{C}$ \\
\hline 152 & 123639.58 & +621230.5 & 0.6 & $80.9 \pm 10.9$ & $67.1 \pm 9.7$ & $18.1 \pm 6.2$ & $<6.6$ & $0.27_{-0.10}^{+0.10}$ & 1.87 & 923.5 & 0.69 & 0.33 & 0.38 & $<0.23$ & $\mathrm{H}$ \\
\hline 153 & 123639.65 & +620936.7 & 0.6 & $14.3 \pm 6.9^{\mathrm{b}}$ & $9.5 \pm 5.3^{\mathrm{a}}$ & $<12.1$ & $<4.8$ & $<1.29$ & $1.40^{\mathrm{d}}$ & 764.6 & 0.19 & 0.06 & $<0.33$ & $<0.22$ & $\mathrm{C}$ \\
\hline 154 & 123639.69 & +621010.0 & 0.6 & $82.6 \pm 11.3$ & $51.8 \pm 8.8$ & $28.1 \pm 7.7$ & $<13.2$ & $0.54_{-0.17}^{+0.19}$ & 1.39 & 870.4 & 0.98 & 0.26 & 0.68 & $<0.52$ & $\mathrm{C}$ \\
\hline
\end{tabular}


Table 3-Continued

\begin{tabular}{|c|c|c|c|c|c|c|c|c|c|c|c|c|c|c|c|}
\hline \multirow[b]{2}{*}{$\begin{array}{l}\# \\
(1)\end{array}$} & \multicolumn{2}{|c|}{ Coordinates } & \multirow{2}{*}{$\begin{array}{c}\text { Positional } \\
\text { Error } \\
(4)\end{array}$} & \multicolumn{4}{|c|}{ Counts } & \multirow{2}{*}{$\begin{array}{l}\text { Band } \\
\text { Ratio } \\
(9)\end{array}$} & \multirow{2}{*}{$\begin{array}{c}\text { Effective } \\
\Gamma \\
(10)\end{array}$} & \multirow{2}{*}{$\begin{array}{c}\text { Exposure } \\
\text { Exposure }(\mathrm{ks}) \\
(11)\end{array}$} & \multirow[b]{2}{*}{$\begin{array}{l}\text { FB } \\
(12)\end{array}$} & \multicolumn{3}{|c|}{ Flux } & \multirow[b]{2}{*}{$\begin{array}{l}\text { Notes } \\
(16)\end{array}$} \\
\hline & $\begin{array}{c}\alpha_{2000} \\
(2)\end{array}$ & $\begin{array}{c}\delta_{2000} \\
(3)\end{array}$ & & $\begin{array}{l}\text { FB } \\
(5)\end{array}$ & $\begin{array}{l}\text { SB } \\
(6)\end{array}$ & $\begin{array}{l}\mathrm{HB} \\
(7)\end{array}$ & $\begin{array}{l}\text { UHB } \\
(8)\end{array}$ & & & & & $\begin{array}{l}\text { SB } \\
(13)\end{array}$ & $\begin{array}{c}\mathrm{HB} \\
(14)\end{array}$ & $\begin{array}{l}\text { UHB } \\
(15)\end{array}$ & \\
\hline 155 & 123640.00 & +621250.2 & 0.6 & $24.3 \pm 6.9$ & $14.5 \pm 5.4$ & $11.9 \pm 5.4^{\mathrm{b}}$ & $<8.8$ & $0.82_{-0.44}^{+0.61}$ & $1.40^{\mathrm{d}}$ & 921.4 & 0.27 & 0.07 & 0.27 & $<0.33$ & $\mathrm{C}$ \\
\hline 156 & $1236 \quad 40.14$ & +621655.8 & 0.6 & $19.2 \pm 6.8$ & $20.5 \pm 6.2$ & $<8.4$ & $<8.1$ & $<0.42$ & $1.40^{\mathrm{d}}$ & 770.8 & 0.26 & 0.12 & $<0.23$ & $<0.37$ & $\mathrm{C}$ \\
\hline 157 & 123640.14 & +621940.8 & 0.7 & $57.1 \pm 11.6$ & $58.8 \pm 10.0$ & $<15.4$ & $<14.3$ & $<0.26$ & $>1.88$ & 835.2 & 0.53 & 0.32 & $<0.35$ & $<0.57$ & \\
\hline 158 & 123640.79 & +621041.4 & 0.6 & $133.2 \pm 13.8$ & $61.8 \pm 9.5$ & $69.2 \pm 10.4$ & $31.9 \pm 7.8$ & $1.12_{-0.23}^{+0.27}$ & 0.69 & 921.4 & 2.19 & 0.29 & 1.85 & 1.31 & $\mathrm{C}, \mathrm{NP}$ \\
\hline 159 & 123641.58 & +620948.1 & 0.6 & $41.9 \pm 9.0$ & $<10.3$ & $35.9 \pm 8.4$ & $30.1 \pm 7.8$ & $>3.51$ & $<-0.26$ & 762.0 & 1.33 & $<0.06$ & 1.45 & 1.69 & C \\
\hline 160 & 123641.80 & +621132.0 & 0.6 & $32.9 \pm 7.9$ & $25.5 \pm 6.6$ & $<10.8$ & $<10.3$ & $<0.43$ & $1.40^{\mathrm{d}}$ & 880.8 & 0.38 & 0.13 & $<0.26$ & $<0.41$ & $\mathrm{H}$ \\
\hline 161 & 123642.11 & +621331.6 & 0.6 & $22.7 \pm 6.8$ & $20.4 \pm 6.0$ & $<9.5$ & $<11.3$ & $<0.47$ & $1.40^{\mathrm{d}}$ & 940.5 & 0.25 & 0.10 & $<0.21$ & $<0.42$ & $\mathrm{C}$ \\
\hline 162 & 123642.21 & $+6217 \quad 11.8$ & 0.6 & $222.0 \pm 17.2$ & $155.4 \pm 14.0$ & $70.9 \pm 10.6$ & $23.9 \pm 7.0$ & $0.46_{-0.08}^{+0.08}$ & 1.52 & 712.4 & 2.98 & 0.97 & 2.07 & 1.17 & $\mathrm{C}$ \\
\hline 163 & 123642.22 & +621545.8 & 0.6 & $189.1 \pm 15.9$ & $122.0 \pm 12.5$ & $72.9 \pm 10.4$ & $34.9 \pm 7.8$ & $0.61_{-0.10}^{+0.11}$ & 1.29 & 644.4 & 3.20 & 0.84 & 2.48 & 1.98 & $\mathrm{C}$ \\
\hline 164 & 123642.30 & +620612.1 & 0.9 & $108.3 \pm 15.1^{\mathrm{c}}$ & $62.3 \pm 10.6$ & $48.0 \pm 11.7$ & $<23.8$ & $0.78_{-0.25}^{+0.15}$ & 1.06 & 677.8 & 1.98 & 0.40 & 1.61 & $<1.31$ & $\mathrm{O}$ \\
\hline 165 & 123644.35 & +621133.1 & 0.6 & $18.9 \pm 6.6$ & $17.5 \pm 5.8$ & $<7.7$ & $<3.9$ & $\begin{array}{l}<0.44 \\
<0.22\end{array}$ & $1.40^{\mathrm{d}}$ & 920.2 & 0.21 & 0.08 & $<0.18$ & $<0.15$ & $\mathrm{H}$ \\
\hline 166 & $1236 \quad 45.07$ & +620308.8 & 1.3 & $19.7 \pm 10.2$ & $17.8 \pm 8.0$ & $<16.7$ & $<13.4$ & $<0.95$ & $1.40^{\mathrm{d}}$ & 93.9 & 2.14 & 0.85 & $<3.81$ & $<5.29$ & \\
\hline 167 & 123645.51 & +621850.5 & 0.6 & $192.9 \pm 16.7$ & $154.2 \pm 14.2$ & $43.1 \pm 9.4$ & $<15.1$ & $0.28_{-0.07}^{+0.07}$ & 1.85 & 892.1 & 1.71 & 0.78 & 0.93 & $<0.56$ & I \\
\hline 168 & 123645.88 & +620754.1 & 0.7 & $17.6 \pm 9.1^{b c}$ & $19.4 \pm 7.3$ & $<16.8$ & $<14.3$ & $\begin{array}{l}<0.87 \\
<0.87\end{array}$ & $1.40^{\mathrm{d}}$ & 878.6 & 0.20 & 0.10 & $<0.40$ & $<0.57$ & $a$ \\
\hline 169 & 123646.01 & +622145.4 & 0.9 & $88.9 \pm 15.0$ & $73.8 \pm 12.0$ & $<26.3$ & $<23.0$ & $<0.36$ & $>1.70$ & 693.3 & 1.11 & 0.48 & $<0.75$ & $<1.14$ & I \\
\hline 170 & 123646.05 & +621449.0 & 0.6 & $12.6 \pm 5.7^{\mathrm{b}}$ & $9.2 \pm 4.6$ & $<8.8$ & $<5.9$ & $<0.98$ & $1.40^{\mathrm{d}}$ & 935.9 & 0.14 & 0.04 & $<0.20$ & $<0.22$ & $\mathrm{C}, \mathrm{NP}$ \\
\hline 171 & 123646.35 & +621404.8 & 0.6 & $1311.4 \pm 37.4$ & $592.8 \pm 25.6$ & $730.6 \pm 28.4$ & $244.9 \pm 17.2$ & $1.25_{-0.07}^{+0.07}$ & 0.58 & 923.1 & 22.79 & 2.80 & 20.12 & 10.29 & $\mathrm{H}$ \\
\hline 172 & 123646.41 & +621529.2 & 0.6 & $39.3 \pm 8.2$ & $25.6 \pm 6.5$ & $14.8 \pm 5.9^{\mathrm{b}}$ & $6.4 \pm 4.7^{\mathrm{b}}$ & $0.59^{+0.31}$ & 1.32 & 798.0 & 0.53 & 0.14 & 0.40 & 0.29 & $\mathrm{C}$ \\
\hline 173 & 123646.57 & +620857.3 & 0.6 & $22.4 \pm 8.3$ & $<11.8$ & $19.0 \pm 7.6$ & $<13.3$ & $>1.61$ & $<0.33$ & 881.0 & 0.46 & $<0.06$ & 0.58 & $<0.60$ & $\mathrm{C}$ \\
\hline 174 & 123647.93 & +620941.3 & 0.6 & $90.2 \pm 11.9$ & $60.4 \pm 9.5$ & $28.6 \pm 7.9$ & $<11.8$ & $0.47_{-0.15}^{+0.16}$ & 1.50 & 871.5 & 1.00 & 0.31 & 0.67 & $<0.46$ & C \\
\hline 175 & 123647.95 & +621019.9 & 0.6 & $36.2 \pm 8.3$ & $11.6 \pm 5.3$ & $24.4 \pm 7.2$ & $<11.2$ & $2.11_{-0.91}^{+1.87}$ & 0.10 & 771.0 & 0.96 & 0.07 & 0.89 & $<0.59$ & $\mathrm{C}$ \\
\hline 176 & 123648.05 & +621309.1 & 0.6 & $60.8 \pm 9.7$ & $38.8 \pm 7.7$ & $26.7 \pm 7.1$ & $13.6 \pm 5.7^{\mathrm{b}}$ & $0.69_{-0.22}^{+0.25}$ & 1.17 & 945.0 & 0.75 & 0.18 & 0.63 & 0.51 & $\mathrm{H}$ \\
\hline 177 & 123648.30 & +621456.3 & 0.6 & $66.4 \pm 10.1$ & $30.4 \pm 6.9$ & $36.1 \pm 7.9$ & $11.8 \pm 5.4^{\mathrm{b}}$ & $1.21_{-0.35}^{+0.44}$ & 0.61 & 935.6 & 1.12 & 0.14 & 0.98 & 0.49 & $\mathrm{C}$ \\
\hline 178 & 123648.38 & +621426.2 & 0.6 & $13.6 \pm 5.7^{\mathrm{b}}$ & $13.4 \pm 5.3$ & $<7.7$ & $<8.8$ & $\begin{array}{l}-0.35 \\
<0.58\end{array}$ & $1.40^{\mathrm{d}}$ & 941.8 & 0.15 & 0.06 & $<0.17$ & $<0.33$ & $\mathrm{H}$ \\
\hline 179 & 123648.87 & $\begin{array}{r}+622155.8\end{array}$ & 0.6 & $41.7 \pm 8.8$ & $<17.5$ & $45.1 \pm 9.8$ & $<24.0$ & $>2.63$ & $<-0.06$ & 684.7 & 1.34 & $<0.11$ & 1.95 & $<1.51$ & $\mathrm{M}$ \\
\hline 180 & 123648.94 & +620325.2 & 1.2 & $106.3 \pm 15.9$ & $72.1 \pm 11.8$ & $33.2 \pm 11.4^{\mathrm{bc}}$ & $<21.6$ & $0.46_{-0.17}^{+0.18}$ & 1.52 & 333.1 & 3.05 & 0.98 & 2.08 & $<2.35$ & $\mathrm{O}$ \\
\hline 181 & 123649.17 & +620439.2 & 1.1 & $62.2 \pm 14.0$ & $40.3 \pm 10.1^{\mathrm{b}}$ & $<25.8$ & $<20.7$ & $<0.65$ & $>1.23$ & 365.4 & 1.92 & 0.48 & $<1.56$ & $<2.08$ & \\
\hline 182 & 123649.35 & +621849.6 & 0.6 & $21.9 \pm 8.3$ & $11.4 \pm 5.9^{\mathrm{b}}$ & $12.6 \pm 7.0^{\mathrm{b}}$ & $<14.4$ & $1.12_{-0}^{+1.35}$ & $1.40^{\mathrm{d}}$ & 877.8 & 0.26 & 0.06 & 0.30 & $<0.57$ & \\
\hline 183 & 123649.45 & +621347.2 & 0.6 & $7.8 \pm 4.9^{\mathrm{a}}$ & $10.0 \pm 4.8^{\mathrm{a}}$ & $<7.7$ & $<4.2$ & $<0.78$ & $1.40^{\mathrm{d}}$ & 909.2 & 0.09 & 0.05 & $<0.18$ & $<0.16$ & $\mathrm{H}$ \\
\hline 184 & 123649.67 & +620737.9 & 0.7 & $1664.3 \pm 43.7$ & $869.9 \pm 31.6$ & $811.8 \pm 31.5$ & $264.2 \pm 19.8$ & $0.94_{-0.05}^{+0.05}$ & 0.87 & 855.8 & 26.69 & 4.45 & 22.41 & 11.50 & \\
\hline 185 & 123649.76 & +621313.0 & 0.6 & $13.9 \pm 5.8^{\mathrm{b}}$ & $13.8 \pm 5.3$ & $<7.7$ & $<5.6$ & $<0.56$ & $1.40^{\mathrm{d}}$ & 945.2 & 0.15 & 0.06 & $<0.17$ & $<0.21$ & $\mathrm{H}$ \\
\hline 186 & $12 \quad 36 \quad 50.34$ & $+62 \quad 2146.3$ & 0.9 & $347.8 \pm 23.2$ & $258.0 \pm 18.7$ & $97.9 \pm 14.7$ & $<26.2$ & $0.39_{-0.06}^{+0.07}$ & 1.65 & 731.9 & 4.21 & 1.58 & 2.70 & $<1.25$ & \\
\hline 187 & 123650.41 & +622005.4 & 0.7 & $159.3 \pm 16.4$ & $121.6 \pm 13.3$ & $47.3 \pm 10.9^{c}$ & $<19.0$ & $0.40^{+0.10}$ & 1.64 & 866.0 & 1.64 & 0.63 & 1.10 & $<0.76$ & $\mathrm{O}$ \\
\hline 188 & 123651.11 & +621030.7 & 0.6 & $24.3 \pm 7.3$ & $18.1 \pm 6.0$ & $<10.5$ & $<5.7$ & $<0.58$ & $1.40^{\mathrm{d}}$ & 839.2 & 0.30 & 0.10 & $<0.26$ & $<0.23$ & C \\
\hline 189 & 123651.25 & +621051.6 & 0.6 & $41.0 \pm 8.6$ & $<12.7$ & $36.0 \pm 8.0$ & $12.6 \pm 5.7^{\mathrm{b}}$ & $>2.85$ & $<-0.11$ & 727.5 & 1.27 & $<0.08$ & 1.47 & 0.72 & C \\
\hline 190 & $1236 \quad 51.75$ & +621221.4 & 0.6 & $148.1 \pm 14.2$ & $60.1 \pm 9.2$ & $91.9 \pm 11.6$ & $24.2 \pm 6.8$ & $1.54_{-0.28}^{+0.34}$ & 0.37 & 941.2 & 2.81 & 0.28 & 2.59 & 1.02 & $\mathrm{H}$ \\
\hline 191 & $12 \quad 3651.83$ & +621504.9 & 0.6 & $165.7 \pm 14.9$ & $110.5 \pm 12.0$ & $56.3 \pm 9.3$ & $21.4 \pm 6.6$ & $0.52_{-0.10}^{+0.11}$ & 1.43 & 782.5 & 2.13 & 0.63 & 1.52 & 0.96 & $\mathrm{C}, \mathrm{NP}$ \\
\hline 192 & $12 \quad 36 \quad 52.17$ & $+62 \quad 2810.9$ & 1.7 & $2778.6 \pm 60.5$ & $2136.2 \pm 50.1$ & $633.7 \pm 34.4^{\mathrm{c}}$ & $<44.2$ & $0.35_{-0.02}^{+0.02}$ & 1.72 & 178.0 & 133.08 & 53.01 & 80.43 & $<11.28$ & $\mathrm{O}$ \\
\hline
\end{tabular}


Table 3-Continued

\begin{tabular}{|c|c|c|c|c|c|c|c|c|c|c|c|c|c|c|c|}
\hline \multirow[b]{2}{*}{$\begin{array}{l}\# \\
(1)\end{array}$} & \multicolumn{2}{|c|}{ Coordinates } & \multirow{2}{*}{$\begin{array}{l}\text { Positional } \\
\text { Error } \\
(4)\end{array}$} & \multirow[b]{2}{*}{$\begin{array}{l}\text { FB } \\
(5)\end{array}$} & \multicolumn{2}{|c|}{ Counts } & \multirow[b]{2}{*}{$\begin{array}{l}\text { UHB } \\
(8)\end{array}$} & \multirow{2}{*}{$\begin{array}{c}\text { Band } \\
\text { Ratio } \\
\text { (9) }\end{array}$} & \multirow{2}{*}{$\begin{array}{c}\text { Effective } \\
\Gamma \\
(10)\end{array}$} & \multirow{2}{*}{$\begin{array}{c}\text { Exposure } \\
\text { Exposure }(\mathrm{ks}) \\
\quad(11)\end{array}$} & \multirow[b]{2}{*}{$\begin{array}{l}\text { FB } \\
(12)\end{array}$} & \multicolumn{2}{|c|}{ Flux } & \multirow[b]{2}{*}{$\begin{array}{l}\text { UHB } \\
(15)\end{array}$} & \multirow[b]{2}{*}{$\begin{array}{l}\text { Notes } \\
(16)\end{array}$} \\
\hline & $\begin{array}{c}\alpha_{2000} \\
(2)\end{array}$ & $\begin{array}{c}\delta_{2000} \\
(3)\end{array}$ & & & $\begin{array}{l}\text { SB } \\
(6)\end{array}$ & $\begin{array}{l}\mathrm{HB} \\
(7)\end{array}$ & & & & & & $\begin{array}{l}\mathrm{SB} \\
(13)\end{array}$ & $\begin{array}{l}\mathrm{HB} \\
(14)\end{array}$ & & \\
\hline 193 & 123652.91 & +621444.2 & 0.6 & $58.4 \pm 9.6$ & $49.5 \pm 8.5$ & $11.9 \pm 5.4$ & $<11.2$ & $0.24_{-0.12}^{+0.12}$ & 1.92 & 777.2 & 0.57 & 0.29 & 0.29 & $<0.47$ & $\mathrm{C}$ \\
\hline 194 & $12 \quad 36 \quad 53.41$ & +621139.6 & 0.6 & $19.7 \pm 6.6$ & $18.7 \pm 5.9$ & $<6.7$ & $<4.5$ & $<0.36$ & $1.40^{\mathrm{d}}$ & 935.6 & 0.22 & 0.09 & $<0.15$ & $<0.17$ & $\mathrm{C}$ \\
\hline 195 & 123653.56 & +622018.8 & 0.8 & $43.2 \pm 11.7$ & $<16.6$ & $33.2 \pm 10.3^{\mathrm{a}}$ & $<20.7$ & $>2.04$ & $<0.13$ & 862.4 & 1.01 & $<0.08$ & 1.09 & $<1.01$ & \\
\hline 196 & 123653.63 & $+6211 \quad 15.5$ & 0.6 & $<9.9$ & $5.5 \pm 4.2^{\mathrm{a}}$ & $<3.9$ & $<4.1$ & $<0.72$ & $1.40^{\mathrm{d}}$ & 731.1 & $<0.14$ & 0.03 & $<0.11$ & $<0.19$ & $\mathrm{C}$ \\
\hline 197 & 123654.57 & +621111.0 & 0.6 & $30.7 \pm 7.7$ & $12.1 \pm 5.2$ & $20.6 \pm 6.7$ & $10.6 \pm 5.4^{\mathrm{a}}$ & $1.70_{-0.75}^{+1.38}$ & 0.28 & 744.4 & 0.77 & 0.07 & 0.75 & 0.56 & $\mathrm{C}$ \\
\hline 198 & 123655.46 & +621311.4 & 0.6 & $122.2 \pm 13.0$ & $84.1 \pm 10.6$ & $39.7 \pm 8.2$ & $10.3 \pm 5.2$ & $0.48_{-0.11}^{+0.12}$ & 1.50 & 929.9 & 1.27 & 0.40 & 0.89 & 0.38 & $\mathrm{H}$ \\
\hline 199 & 123655.80 & +621200.7 & 0.6 & $39.0 \pm 8.2$ & $27.0 \pm 6.7$ & $15.0 \pm 6.0^{\mathrm{b}}$ & $<10.1$ & $0.56_{-0.25}^{+0.29}$ & 1.36 & 938.6 & 0.43 & 0.13 & 0.34 & $<0.38$ & $\mathrm{C}$ \\
\hline 200 & 123655.86 & +620807.9 & 0.7 & $27.0 \pm 9.6^{\mathrm{b}}$ & $24.2 \pm 7.5$ & $<16.4$ & $<14.0$ & $<0.68$ & $1.40^{\mathrm{d}}$ & 872.3 & 0.32 & 0.12 & $<0.39$ & $<0.56$ & \\
\hline 201 & 123656.58 & +621513.2 & 0.6 & $24.3 \pm 6.9$ & $<7.7$ & $23.2 \pm 6.8$ & $10.3 \pm 5.2$ & $>3.09$ & $<-0.17$ & 945.2 & 0.60 & $<0.04$ & 0.75 & 0.47 & $\mathrm{C}$ \\
\hline 202 & 123656.64 & +621245.4 & 0.6 & $10.1 \pm 5.3$ & $<13.8$ & $<11.3$ & $<8.1$ & - & $1.40^{\mathrm{d}}$ & 916.9 & 0.11 & $<0.07$ & $<0.26$ & $<0.31$ & $\mathrm{H}$ \\
\hline 203 & 123656.93 & +621301.6 & 0.6 & $96.0 \pm 11.8$ & $73.3 \pm 10.0$ & $23.2 \pm 6.8$ & $7.2 \pm 4.8^{\mathrm{b}}$ & $0.32_{-0.10}^{+0.11}$ & 1.77 & 922.5 & 0.86 & 0.36 & 0.49 & 0.26 & $\mathrm{H}$ \\
\hline 204 & $1236 \quad 56.90$ & +620458.6 & 1.1 & $251.1 \pm 20.8$ & $180.2 \pm 16.2$ & $67.1 \pm 13.6$ & $<24.1$ & $0.38_{-0.08}^{+0.09}$ & 1.67 & 493.5 & 4.47 & 1.64 & 2.73 & $<1.70$ & \\
\hline 205 & $1236 \quad 57.34$ & +621024.8 & 0.6 & $23.0 \pm 7.3$ & $17.6 \pm 6.0$ & $<11.8$ & $<8.7$ & $<0.67$ & $1.40^{\mathrm{d}}$ & 870.8 & 0.27 & 0.09 & $<0.28$ & $<0.34$ & $\mathrm{C}$ \\
\hline 206 & $12 \quad 3657.48$ & $\begin{array}{lll}62 & 12 & 10.1\end{array}$ & 0.6 & $22.2 \pm 6.8$ & $8.9 \pm 4.6^{\mathrm{a}}$ & $12.8 \pm 5.8^{\mathrm{b}}$ & $<9.1$ & $1.44_{-0.82}^{+1.68}$ & $1.40^{\mathrm{d}}$ & 939.2 & 0.24 & 0.04 & 0.29 & $<0.34$ & $\mathrm{H}$ \\
\hline 207 & 123657.61 & +620314.3 & 1.3 & $67.2 \pm 15.7$ & $53.5 \pm 11.3$ & $<28.7$ & $<23.3$ & $<0.55$ & $>1.38$ & 354.4 & 1.96 & 0.67 & $<1.73$ & $<2.42$ & \\
\hline 208 & 123657.90 & +622128.1 & 0.9 & $420.6 \pm 25.1$ & $302.6 \pm 20.0$ & $122.3 \pm 15.7$ & $45.4 \pm 11.3^{\mathrm{c}}$ & $0.41_{-0.06}^{+0.06}$ & 1.61 & 787.2 & 4.86 & 1.72 & 3.17 & 2.04 & $\mathrm{O}$ \\
\hline 209 & 123658.42 & +620958.5 & 0.6 & $20.0 \pm 7.3^{\mathrm{a}}$ & $20.4 \pm 6.4$ & $<11.3$ & $<10.6$ & $<0.56$ & $1.40^{\mathrm{d}}$ & 880.6 & 0.23 & 0.10 & $<0.27$ & $<0.41$ & $\mathrm{C}$ \\
\hline 210 & 123658.63 & +620403.3 & 1.2 & $386.7 \pm 24.4$ & $285.4 \pm 19.7$ & $95.7 \pm 14.9$ & $35.4 \pm 10.9$ & $0.34_{-0.06}^{+0.06}$ & 1.74 & 378.5 & 8.63 & 3.40 & 5.01 & 3.25 & \\
\hline 211 & 123658.83 & +621022.3 & 0.6 & $42.6 \pm 8.9$ & $<10.3$ & $39.9 \pm 8.6$ & $<13.0$ & $>3.87$ & $<-0.34$ & 882.5 & 1.20 & $<0.05$ & 1.41 & $<0.63$ & $\mathrm{C}$ \\
\hline 212 & 123658.85 & +621434.9 & 0.6 & $697.3 \pm 29.1$ & $482.0 \pm 23.7$ & $231.2 \pm 17.3$ & $78.7 \pm 10.8$ & $0.49_{-0.04}^{+0.04}$ & 1.48 & 932.4 & 7.31 & 2.30 & 5.17 & 2.92 & $\mathrm{C}$ \\
\hline 213 & 123659.20 & +621832.3 & 0.6 & $40.8 \pm 9.4$ & $19.0 \pm 6.5$ & $22.8 \pm 7.7$ & $<12.9$ & $1.22_{-0.51}^{+0.75}$ & 0.61 & 746.0 & 0.86 & 0.11 & 0.77 & $<0.68$ & \\
\hline 214 & 123659.19 & +622523.3 & 1.4 & $503.4 \pm 30.4$ & $376.4 \pm 23.3$ & $118.6 \pm 19.8$ & $<36.3$ & $0.33_{-0.06}^{+0.06}$ & 1.75 & 438.8 & 9.60 & 3.84 & 5.48 & $<3.09$ & NP \\
\hline 215 & 123659.81 & +621933.4 & 0.7 & $168.6 \pm 16.3$ & $134.6 \pm 13.7$ & $46.3 \pm 10.4$ & $23.9 \pm 8.3^{\mathrm{b}}$ & $\begin{array}{l}0.35_{-0.09}^{+0.09} \\
\end{array}$ & 1.72 & 863.9 & 1.66 & 0.70 & 1.07 & 0.95 & \\
\hline 216 & 123700.46 & +621508.9 & 0.6 & $99.1 \pm 11.9$ & $60.3 \pm 9.3$ & $43.9 \pm 8.6$ & $14.9 \pm 5.8$ & $0.74_{-0.17}^{+0.20}$ & 1.11 & 720.0 & 1.66 & 0.37 & 1.38 & 0.75 & C \\
\hline 217 & 123701.18 & +620627.7 & 0.9 & $171.3 \pm 17.8$ & $49.6 \pm 10.2$ & $122.6 \pm 15.3$ & $58.3 \pm 11.6$ & $2.50_{-0.53}^{+0.72}$ & -0.03 & 848.6 & 4.37 & 0.25 & 4.23 & 2.94 & \\
\hline 218 & 123701.50 & +621847.3 & 0.6 & $24.4 \pm 8.6^{\mathrm{b}}$ & $21.4 \pm 6.8$ & $<12.4$ & $<10.4$ & $<0.59$ & $1.40^{\mathrm{d}}$ & 788.9 & 0.32 & 0.12 & $<0.33$ & $<0.47$ & \\
\hline 219 & $\begin{array}{lll}12 & 37 & 01.73\end{array}$ & +620720.6 & 0.8 & $37.1 \pm 11.2$ & $<15.6$ & $31.2 \pm 10.1$ & $<19.7$ & $>2.03$ & $<0.13$ & 848.5 & 0.88 & $<0.08$ & 1.04 & $<0.96$ & \\
\hline 220 & 123702.04 & +621122.4 & 0.6 & $13.8 \pm 6.1^{\mathrm{b}}$ & $11.3 \pm 5.1$ & $<8.1$ & $<4.9$ & $<0.72$ & $1.40^{\mathrm{d}}$ & 918.1 & 0.15 & 0.05 & $<0.18$ & $<0.18$ & $\mathrm{C}$ \\
\hline 221 & 123702.39 & +621925.8 & 0.7 & $241.0 \pm 18.8$ & $142.4 \pm 14.0$ & $99.3 \pm 13.1$ & $25.4 \pm 8.3^{\mathrm{a}}$ & $0.71_{-0.11}^{+0.12}$ & 1.15 & 803.0 & 3.54 & 0.78 & 2.78 & 1.17 & NP \\
\hline 222 & 123702.60 & +621244.0 & 0.6 & $32.1 \pm 7.7$ & $22.8 \pm 6.3$ & $<10.8$ & $<10.3$ & $<0.48$ & $1.40^{\mathrm{d}}$ & 939.4 & 0.35 & 0.11 & $<0.24$ & $<0.39$ & C \\
\hline 223 & 123702.71 & +621543.7 & 0.6 & $1668.0 \pm 42.2$ & $886.7 \pm 31.0$ & $792.7 \pm 29.6$ & $285.3 \pm 18.5$ & $0.90_{-0.05}^{+0.05}$ & 0.92 & 903.2 & 24.74 & 4.30 & 20.51 & 11.49 & $\mathrm{C}$ \\
\hline 224 & 123702.82 & +621601.3 & 0.6 & $193.4 \pm 16.1$ & $134.7 \pm 13.2$ & $65.8 \pm 10.2$ & $26.9 \pm 7.2$ & $0.49_{-0.09}^{+0.09}$ & 1.48 & 901.1 & 2.10 & 0.67 & 1.51 & 1.01 & $\mathrm{C}$ \\
\hline 225 & 123703.56 & +621632.4 & 0.6 & $38.0 \pm 8.4$ & $<11.8$ & $28.3 \pm 7.6$ & $<13.3$ & $>2.40$ & $<0.00$ & 929.8 & 0.87 & $<0.06$ & 0.88 & $<0.59$ & C \\
\hline 226 & $12 \quad 3704.08$ & +621155.2 & 0.6 & $14.3 \pm 6.0$ & $<9.7$ & $11.2 \pm 5.6^{\mathrm{b}}$ & $<10.5$ & $>1.16$ & $1.40^{\mathrm{d}}$ & 918.2 & 0.16 & $<0.05$ & 0.26 & $<0.39$ & $\mathrm{C}, \mathrm{NP}$ \\
\hline 227 & 123704.08 & +620755.0 & 0.7 & $634.2 \pm 28.6$ & $472.3 \pm 23.8$ & $160.2 \pm 15.8$ & $48.2 \pm 10.0$ & $0.34_{-0.04}^{+0.04}$ & 1.73 & 875.4 & 6.13 & 2.43 & 3.60 & 1.86 & \\
\hline 228 & 123704.62 & +621652.0 & 0.6 & $181.4 \pm 15.9$ & $135.9 \pm 13.4$ & $50.7 \pm 9.4$ & $14.1 \pm 6.2^{\mathrm{b}}$ & $0.37_{-0.08}^{+0.08}$ & 1.67 & 921.5 & 1.72 & 0.66 & 1.09 & 0.51 & $\mathrm{C}$ \\
\hline 229 & 123704.66 & +621429.0 & 0.6 & $20.3 \pm 6.6$ & $<10.0$ & $12.7 \pm 5.7^{\mathrm{b}}$ & $<9.5$ & $>1.29$ & $1.40^{\mathrm{d}}$ & 922.4 & 0.23 & $<0.05$ & 0.29 & $<0.36$ & $\mathrm{C}$ \\
\hline 230 & 123704.86 & +621601.6 & 0.6 & $647.9 \pm 28.1$ & $416.0 \pm 22.1$ & $249.6 \pm 18.0$ & $92.9 \pm 11.7$ & $0.60_{-0.05}^{+0.06}$ & 1.30 & 902.1 & 7.77 & 2.04 & 5.94 & 3.58 & $\mathrm{C}$ \\
\hline
\end{tabular}


Table 3-Continued

\begin{tabular}{|c|c|c|c|c|c|c|c|c|c|c|c|c|c|c|c|c|}
\hline \multirow[b]{2}{*}{$\begin{array}{l}\# \\
(1)\end{array}$} & \multicolumn{2}{|c|}{ Coordinates } & \multirow{2}{*}{$\begin{array}{l}\text { Positional } \\
\text { Error } \\
(4)\end{array}$} & \multicolumn{4}{|c|}{ Counts } & \multirow{2}{*}{$\begin{array}{c}\text { Band } \\
\text { Ratio } \\
\text { (9) }\end{array}$} & \multirow{2}{*}{$\begin{array}{c}\text { Effective } \\
\Gamma \\
(10)\end{array}$} & \multirow{2}{*}{$\begin{array}{c}\text { Exposure } \\
\text { Exposure (ks) } \\
(11)\end{array}$} & \multicolumn{4}{|c|}{ Flux } & \multirow{2}{*}{\multicolumn{2}{|c|}{$\begin{array}{c}\text { Notes } \\
(16)\end{array}$}} \\
\hline & $\begin{array}{c}\alpha_{2000} \\
(2)\end{array}$ & $\begin{array}{c}\delta_{2000} \\
(3)\end{array}$ & & $\begin{array}{l}\text { FB } \\
(5)\end{array}$ & $\begin{array}{l}\text { SB } \\
(6)\end{array}$ & $\begin{array}{l}\mathrm{HB} \\
(7)\end{array}$ & $\begin{array}{l}\text { UHB } \\
(8)\end{array}$ & & & & $\begin{array}{l}\text { FB } \\
(12)\end{array}$ & $\begin{array}{l}\text { SB } \\
(13)\end{array}$ & $\begin{array}{c}\text { HB } \\
(14)\end{array}$ & $\begin{array}{l}\text { UHB } \\
(15)\end{array}$ & & \\
\hline 231 & 123705.10 & +621634.8 & 0.6 & $87.2 \pm 11.7$ & $43.9 \pm 8.3$ & $42.8 \pm 8.8$ & $15.8 \pm 6.3$ & $0.98_{-0.25}^{+0.30}$ & 0.83 & 929.1 & 1.32 & 0.21 & 1.10 & 0.63 & $\mathrm{C}$ & \\
\hline 232 & 123705.96 & +620131.1 & 1.5 & $201.7 \pm 20.7$ & $113.2 \pm 14.1$ & $83.7 \pm 15.3^{\mathrm{bc}}$ & $<29.5$ & $0.76_{-0.16}^{+0.18}$ & 1.08 & 281.7 & 8.76 & 1.77 & 6.86 & $<4.13$ & $\mathrm{O}$ & \\
\hline 233 & 123706.21 & $+62 \quad 2136.2$ & 0.9 & $1266.9 \pm 42.2$ & $983.6 \pm 36.1$ & $289.5 \pm 22.3$ & $91.9 \pm 15.1$ & $0.30_{-0.03}^{+0.03}$ & 1.81 & 823.4 & 12.47 & 5.40 & 6.91 & 3.88 & $\mathrm{~s}$ & \\
\hline 234 & 123706.67 & +622227.6 & 1.0 & $47.8 \pm 16.8^{\mathrm{a}}$ & $<27.1$ & $<36.0$ & $<29.9$ & -0.03 & $1.40^{\mathrm{d}}$ & 785.1 & 0.62 & $<0.15$ & $<0.98$ & $<1.40$ & & \\
\hline 235 & 123706.68 & +622550.1 & 1.4 & $1261.4 \pm 46.9$ & $840.8 \pm 35.7$ & $391.6 \pm 31.7$ & $77.5 \pm 22.1$ & $0.49_{-0.04}^{+0.05}$ & 1.47 & 264.0 & 47.00 & 14.13 & 32.29 & 11.63 & NP & \\
\hline 236 & $1237 \quad 06.81$ & +620722.6 & 0.8 & $147.9 \pm 16.3$ & $76.0 \pm 11.4$ & $76.3 \pm 12.8$ & $27.2 \pm 9.3^{\mathrm{b}}$ & $1.02_{-0.22}^{+0.25}$ & 0.79 & 877.8 & 2.42 & 0.38 & 2.10 & 1.19 & & \\
\hline 237 & 123706.85 & +621702.3 & 0.6 & $3677.9 \pm 62.1$ & $2868.1 \pm 54.8$ & $818.1 \pm 30.3$ & $244.6 \pm 17.6$ & $0.29_{-0.01}^{+0.22}$ & 1.84 & 918.3 & 31.94 & 14.17 & 17.15 & 8.72 & $\mathrm{C}$ & \\
\hline 238 & 123707.06 & +620830.6 & 0.7 & $51.9 \pm 11.0$ & $32.6 \pm 8.1$ & $<19.5$ & $<17.5$ & $<0.60$ & $>1.30$ & 861.1 & 0.65 & 0.17 & $<0.49$ & $<0.72$ & & \\
\hline 239 & 123707.14 & +621642.4 & 0.6 & $7.8 \pm 3.7^{\mathrm{b}}$ & $7.8 \pm 3.3$ & $<5.5$ & $<5.3$ & $<0.71$ & $1.40^{\mathrm{d}}$ & 925.9 & 0.09 & 0.04 & $<0.13$ & $<0.20$ & $\mathrm{C}, \mathrm{M}$ & \\
\hline 240 & 123707.23 & +621408.0 & 0.6 & $54.7 \pm 9.3$ & $24.2 \pm 6.4$ & $32.2 \pm 7.7$ & $14.7 \pm 5.9^{\mathrm{b}}$ & $1.35_{-0.43}^{+0.58}$ & 0.50 & 910.0 & 1.00 & 0.12 & 0.92 & 0.63 & $\mathrm{C}$ & \\
\hline 241 & $1237 \quad 07.47$ & +621955.9 & 0.8 & $118.4 \pm 14.8$ & $86.5 \pm 11.7$ & $38.1 \pm 10.2$ & $<19.3$ & $0.45_{-0.13}^{+0.14}$ & 1.54 & 765.8 & 1.46 & 0.50 & 1.03 & $<0.89$ & & \\
\hline 242 & 123707.49 & +622148.3 & 1.0 & $2239.6 \pm 55.2$ & $1615.8 \pm 45.1$ & $640.7 \pm 34.1$ & $188.3 \pm 22.9$ & $0.41_{-0.02}^{+0.02}$ & 1.62 & 812.9 & 24.91 & 8.89 & 16.11 & 8.26 & $\mathrm{~S}$ & \\
\hline 243 & 123707.66 & +620534.0 & 1.0 & $53.2 \pm 13.0$ & $37.5 \pm 9.4$ & $14.8 \pm 9.9$ & $<20.5$ & $0.40_{-0.28}^{+0.302}$ & 1.63 & 390.7 & 1.22 & 0.43 & 0.76 & $<1.81$ & & 1 \\
\hline 244 & 123707.75 & +621616.2 & 0.6 & $17.7 \pm 6.8$ & $12.2 \pm 5.4^{\mathrm{b}}$ & $<12.2$ & $<9.7$ & $<1.00$ & $1.40^{\mathrm{d}}$ & 933.0 & 0.19 & 0.06 & $<0.27$ & $<0.36$ & $\mathrm{C}$ & $\infty$ \\
\hline 245 & 123707.94 & +621605.8 & 0.6 & $32.1 \pm 8.0$ & $<10.5$ & $23.9 \pm 7.1$ & $12.4 \pm 5.9^{\mathrm{b}}$ & $>2.29$ & $<0.04$ & 930.7 & 0.72 & $<0.05$ & 0.74 & 0.55 & C & । \\
\hline 246 & $\begin{array}{lll}12 & 37 & 08.31\end{array}$ & +621055.9 & 0.6 & $16.4 \pm 6.9^{\mathrm{b}}$ & $13.3 \pm 5.6$ & $<10.6$ & $<9.5$ & $<0.80$ & $1.40^{\mathrm{d}}$ & 927.7 & 0.18 & 0.06 & $<0.24$ & $<0.35$ & $\mathrm{C}$ & \\
\hline 247 & 123708.66 & $\begin{array}{r}621501.5\end{array}$ & 0.6 & $26.7 \pm 7.2$ & $19.0 \pm 5.9$ & $<11.4$ & $<6.7$ & $<0.60$ & $1.40^{\mathrm{d}}$ & 897.7 & 0.30 & 0.09 & $<0.27$ & $<0.26$ & C & \\
\hline 248 & 123708.94 & $\begin{array}{r}620518.4\end{array}$ & 1.0 & $50.7 \pm 13.0$ & $27.3 \pm 8.8$ & $23.6 \pm 10.7$ & $<21.2$ & $0.88^{+0.58}$ & 0.94 & 388.8 & 1.72 & 0.31 & 1.42 & $<2.06$ & & \\
\hline 249 & 123709.30 & +622214.1 & 1.0 & $522.3 \pm 28.9$ & $301.0 \pm 21.0$ & $216.3 \pm 20.6$ & $63.0 \pm 14.0$ & $0.74_{-0.08}^{+0.40}$ & 1.11 & 784.0 & 8.01 & 1.69 & 6.29 & 3.06 & & \\
\hline 250 & 123709.45 & +620837.1 & 0.7 & $50.5 \pm 22.0$ & $24.4 \pm 15.6$ & $10.1 \pm 15.7^{\mathrm{a}}$ & $<17.3$ & $0.42_{-0.67}^{+0.08}$ & 1.60 & 857.6 & 0.54 & 0.13 & 0.24 & $<0.69$ & $\mathrm{~s}$ & \\
\hline 251 & 123709.83 & +620800.8 & 0.8 & $1331.7 \pm 39.7$ & $957.5 \pm 33.1$ & $373.0 \pm 23.4$ & $90.6 \pm 14.5$ & $0.39_{-0.03}^{+0.03}$ & 1.64 & 846.6 & 14.04 & 5.07 & 8.85 & 3.67 & & \\
\hline 252 & 123709.86 & +620833.6 & 0.7 & $21.1 \pm 10.1$ & $<17.8$ & $<21.1$ & $<15.7$ & -0.03 & $1.40^{\mathrm{d}}$ & 851.2 & 0.25 & $<0.09$ & $<0.52$ & $<0.65$ & $\mathrm{~S}$ & \\
\hline 253 & 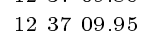 & $\begin{array}{r}+622259.1\end{array}$ & 1.1 & $154.0 \pm 20.7$ & $33.0 \pm 12.0$ & $114.2 \pm 17.7$ & $55.9 \pm 13.9^{\mathrm{a}}$ & $3.57_{-110}^{+2.11}$ & -0.27 & 760.5 & 4.91 & 0.19 & 4.73 & 3.40 & & \\
\hline 254 & $1237 \quad 10.14$ & +620547.3 & 1.0 & $50.0 \pm 12.7$ & $40.3 \pm 9.5$ & $<22.3$ & $<15.1$ & $<0.56$ & $>1.36$ & 392.1 & 1.33 & 0.46 & $<1.22$ & $<1.37$ & & \\
\hline 255 & 123711.03 & $\begin{array}{r}622327.5 \\
\end{array}$ & 1.2 & $375.1 \pm 26.4$ & $300.3 \pm 21.0$ & $88.3 \pm 17.3$ & $<34.7$ & $0.30_{-0.06}^{+0.06}$ & 1.81 & 743.7 & 4.10 & 1.82 & 2.36 & $<1.67$ & & \\
\hline 256 & $1237 \quad 11.17$ & +620713.2 & 0.9 & $154.2 \pm 16.7$ & $101.8 \pm 12.6$ & $51.4 \pm 11.7$ & $<22.7$ & $0.51_{-0.13}^{+0.14}$ & 1.44 & 784.7 & 1.96 & 0.58 & 1.38 & $<1.03$ & & \\
\hline 257 & $12 \quad 37 \quad 11.37$ & +621331.0 & 0.6 & $26.1 \pm 7.2$ & $14.3 \pm 5.4$ & $14.4 \pm 6.0$ & $<10.7$ & $1.02_{-0.51}^{-0.13}$ & $1.40^{\mathrm{d}}$ & 908.1 & 0.29 & 0.07 & 0.34 & $<0.41$ & $\mathrm{C}$ & \\
\hline 258 & $1237 \quad 11.79$ & +621547.6 & 0.6 & $50.7 \pm 9.4$ & $28.4 \pm 7.1$ & $25.4 \pm 7.3$ & $<10.4$ & $0.90_{-0.31}^{+0.39}$ & 0.92 & 929.6 & 0.73 & 0.13 & 0.64 & $<0.41$ & $\mathrm{C}$ & \\
\hline 259 & 123711.97 & +621325.2 & 0.6 & $22.2 \pm 6.9$ & $<9.7$ & $20.4 \pm 6.7$ & $<11.3$ & $>2.14$ & $<0.09$ & 814.9 & 0.56 & $<0.05$ & 0.72 & $<0.57$ & $\mathrm{C}$ & \\
\hline 260 & $12 \quad 37 \quad 12.11$ & $\begin{array}{r}621211.3 \\
+62\end{array}$ & 0.6 & $15.2 \pm 6.3$ & $<9.7$ & $10.3 \pm 5.7^{\mathrm{b}}$ & $<10.8$ & $>1.07$ & $1.40^{\mathrm{d}}$ & 811.3 & 0.19 & $<0.05$ & 0.27 & $<0.46$ & C & \\
\hline 261 & $1237 \quad 12.12$ & +621753.9 & 0.6 & $213.7 \pm 17.3$ & $167.9 \pm 14.7$ & $51.7 \pm 10.0$ & $<16.5$ & $0.31+0.07$ & 1.79 & 905.3 & 1.93 & 0.84 & 1.12 & $<0.61$ & & \\
\hline 262 & 123712.66 & +621342.5 & 0.6 & $13.7 \pm 5.9^{\mathrm{a}}$ & $<8.5$ & $<11.4$ & $8.2 \pm 5.1^{\mathrm{b}}$ & -0.07 & $1.40^{\mathrm{d}}$ & 865.4 & 0.16 & $<0.04$ & $<0.28$ & 0.33 & $\mathrm{C}$ & \\
\hline 263 & $1237 \quad 13.70$ & $\begin{array}{r}+621545.7\end{array}$ & 0.6 & $19.8 \pm 7.1$ & $<9.1$ & $17.7 \pm 6.7^{\mathrm{b}}$ & $13.7 \pm 6.0^{\mathrm{b}}$ & $>1.94$ & $<0.16$ & 929.7 & 0.42 & $<0.04$ & 0.53 & 0.59 & $\mathrm{C}$ & \\
\hline 264 & 123713.81 & +621424.5 & 0.6 & $77.4 \pm 10.9$ & $26.0 \pm 6.7$ & $53.9 \pm 9.3$ & $20.6 \pm 6.6$ & $2.08_{-0.56}^{+0.81}$ & 0.11 & 895.9 & 1.75 & 0.13 & 1.69 & 0.93 & $\mathrm{C}$ & \\
\hline 265 & $1237 \quad 13.89$ & +621458.0 & 0.6 & $27.6 \pm 7.6$ & $<12.0$ & $24.4 \pm 7.1$ & $15.0 \pm 6.0$ & $>2.04$ & $<0.12$ & 862.4 & 0.64 & $<0.06$ & 0.80 & 0.70 & $\mathrm{C}$ & \\
\hline 266 & 123713.92 & +621826.8 & 0.7 & $113.4 \pm 13.9$ & $81.8 \pm 11.1$ & $37.1 \pm 9.4$ & $<16.4$ & $0.46_{-0.13}^{+0.14}$ & 1.52 & 848.9 & 1.27 & 0.43 & 0.90 & $<0.67$ & & \\
\hline 267 & 123714.07 & +620916.7 & 0.7 & $333.2 \pm 21.2$ & $223.7 \pm 16.8$ & $111.2 \pm 13.3$ & $47.1 \pm 9.7$ & $0.50_{-0.07}^{+0.13}$ & 1.46 & 898.9 & 3.67 & 1.11 & 2.58 & 1.83 & $\mathrm{C}$ & \\
\hline 268 & $1237 \quad 14.31$ & +621208.2 & 0.6 & $64.9 \pm 10.3$ & $45.4 \pm 8.3$ & $18.7 \pm 6.7$ & $<10.5$ & $0.42_{-0.16}^{+0.18}$ & 1.60 & 722.0 & 0.82 & 0.28 & 0.52 & $<0.49$ & $\mathrm{C}$ & \\
\hline 269 & $1237 \quad 14.33$ & +621221.0 & 0.6 & $13.0 \pm 6.2^{\mathrm{b}}$ & $11.6 \pm 5.2$ & $<8.7$ & $<7.7$ & $\begin{array}{l}<0.76 \\
<0.16\end{array}$ & $1.40^{\mathrm{d}}$ & 789.5 & 0.17 & 0.07 & $<0.23$ & $<0.34$ & $\mathrm{C}, \mathrm{NP}$ & \\
\hline
\end{tabular}


Table 3-Continued

\begin{tabular}{|c|c|c|c|c|c|c|c|c|c|c|c|c|c|c|c|}
\hline \multirow[b]{2}{*}{$\begin{array}{l}\# \\
(1)\end{array}$} & \multicolumn{2}{|c|}{ Coordinates } & \multirow{2}{*}{$\begin{array}{l}\text { Positional } \\
\text { Error } \\
(4)\end{array}$} & \multirow[b]{2}{*}{$\begin{array}{l}\text { FB } \\
\text { (5) }\end{array}$} & \multicolumn{2}{|c|}{ Counts } & \multirow[b]{2}{*}{$\begin{array}{l}\text { UHB } \\
(8)\end{array}$} & \multirow{2}{*}{$\begin{array}{l}\text { Band } \\
\text { Ratio } \\
(9)\end{array}$} & \multirow{2}{*}{$\begin{array}{c}\text { Effective } \\
\Gamma \\
(10)\end{array}$} & \multirow{2}{*}{$\begin{array}{c}\text { Exposure } \\
\text { Exposure (ks) } \\
(11)\end{array}$} & \multirow[b]{2}{*}{$\begin{array}{l}\text { FB } \\
(12)\end{array}$} & \multicolumn{3}{|c|}{ Flux } & \multirow[b]{2}{*}{$\begin{array}{l}\text { Notes } \\
(16)\end{array}$} \\
\hline & $\begin{array}{c}\alpha_{2000} \\
(2)\end{array}$ & $\begin{array}{c}\delta_{2000} \\
(3)\end{array}$ & & & $\begin{array}{l}\text { SB } \\
(6)\end{array}$ & $\begin{array}{l}\text { HB } \\
(7)\end{array}$ & & & & & & $\begin{array}{l}\text { SB } \\
(13)\end{array}$ & $\begin{array}{l}\mathrm{HB} \\
(14)\end{array}$ & $\begin{array}{l}\text { UHB } \\
(15)\end{array}$ & \\
\hline 270 & 123714.50 & +621839.0 & 0.7 & $20.9 \pm 9.4^{\mathrm{a}}$ & $<13.3$ & $<20.5$ & $<17.7$ & - & $1.40^{\mathrm{d}}$ & 852.1 & 0.25 & $<0.07$ & $<0.51$ & $<0.74$ & \\
\hline 271 & $1237 \quad 14.59$ & +620503.5 & 1.1 & $139.3 \pm 17.0^{\mathrm{c}}$ & $24.4 \pm 8.8$ & $117.6 \pm 15.2$ & $46.1 \pm 11.1^{\mathrm{c}}$ & $4.88_{-1.44}^{+2.84}$ & -0.57 & 370.4 & 10.38 & 0.29 & 10.58 & 5.77 & $\mathrm{O}$ \\
\hline 272 & $1237 \quad 14.75$ & +621616.9 & 0.6 & $300.2 \pm 19.8$ & $203.9 \pm 16.0$ & $101.0 \pm 12.3$ & $32.2 \pm 8.0$ & $0.50_{-0.07}^{+0.07}$ & 1.46 & 924.1 & 3.21 & 0.98 & 2.27 & 1.19 & $\mathrm{C}$ \\
\hline 273 & $1237 \quad 15.24$ & +620231.3 & 1.4 & $275.4 \pm 24.1$ & $165.5 \pm 16.6$ & $97.4 \pm 17.6$ & $51.3 \pm 14.0^{\mathrm{b}}$ & $0.60_{-0.12}^{+0.13}$ & 1.30 & 328.8 & 9.08 & 2.23 & 6.49 & 5.88 & \\
\hline 274 & 123715.96 & +621213.1 & 0.6 & $109.7 \pm 12.7$ & $13.2 \pm 5.5$ & $100.0 \pm 12.1$ & $53.9 \pm 9.4$ & $7.66_{-2.43}^{+5.53}$ & -0.95 & 807.5 & 4.40 & 0.07 & 4.49 & 3.17 & $\mathrm{C}$ \\
\hline 275 & 123715.94 & +620323.8 & 1.3 & $228.3 \pm 22.3$ & $157.7 \pm 16.3$ & $69.1 \pm 15.9^{\mathrm{b}}$ & $<29.6$ & $0.45_{-0.11}^{+0.12}$ & 1.55 & 374.3 & 5.74 & 1.88 & 3.82 & $<2.85$ & \\
\hline 276 & $1237 \quad 16.20$ & +620708.0 & 0.9 & $641.1 \pm 33.8$ & $503.7 \pm 26.1$ & $127.0 \pm 22.5$ & $57.8 \pm 16.3$ & $0.26_{-0.05}^{+0.05}$ & 1.90 & 385.4 & 12.85 & 5.88 & 6.32 & 5.02 & $\mathrm{M}$ \\
\hline 277 & $1237 \quad 16.23$ & +621130.3 & 0.6 & $21.1 \pm 7.3^{\mathrm{b}}$ & $18.8 \pm 6.2$ & $<10.9$ & $<6.9$ & $<0.58$ & $1.40^{\mathrm{d}}$ & 868.0 & 0.25 & 0.10 & $<0.26$ & $<0.28$ & $\mathrm{C}$ \\
\hline 278 & $1237 \quad 16.36$ & $+62 \quad 1512.8$ & 0.6 & $15.7 \pm 6.7^{\mathrm{b}}$ & $15.8 \pm 5.8$ & $<9.9$ & $<7.7$ & $<0.63$ & $1.40^{\mathrm{d}}$ & 886.5 & 0.18 & 0.08 & $<0.24$ & $<0.30$ & $\mathrm{C}$ \\
\hline 279 & $1237 \quad 16.51$ & +621643.2 & 0.6 & $33.7 \pm 16.5$ & $6.6 \pm 9.4^{\mathrm{a}}$ & $25.7 \pm 14.1^{\mathrm{b}}$ & $<13.4$ & $3.93_{-3.16}^{+13.39}$ & -0.35 & 876.6 & 0.97 & 0.03 & 0.92 & $<0.67$ & $\mathrm{C}, \mathrm{M}$ \\
\hline 280 & 123716.66 & +621733.4 & 0.6 & $1449.0 \pm 40.2$ & $801.8 \pm 29.9$ & $652.4 \pm 27.9$ & $176.9 \pm 16.2$ & $0.82_{-0.05}^{+0.05}$ & 1.01 & 906.8 & 20.41 & 3.88 & 16.60 & 7.18 & $\mathrm{C}$ \\
\hline 281 & $1237 \quad 16.79$ & +620623.5 & 1.0 & $111.9 \pm 14.9$ & $32.5 \pm 8.9$ & $74.0 \pm 12.4$ & $25.1 \pm 8.9$ & $2.31_{-0.63}^{+0.96}$ & 0.03 & 395.7 & 5.96 & 0.36 & 5.43 & 2.71 & । \\
\hline 282 & $1237 \quad 16.81$ & +620553.0 & 1.0 & $78.0 \pm 14.0^{\mathrm{c}}$ & $54.5 \pm 10.5$ & $20.6 \pm 10.1^{\mathrm{bc}}$ & $<18.8$ & $0.39_{-0.20}^{+0.21}$ & 1.66 & 363.1 & 1.90 & 0.67 & 1.14 & $<1.81$ & $\mathrm{O}$ \\
\hline 283 & $1237 \quad 17.83$ & +621855.7 & 0.7 & $785.9 \pm 31.6$ & $600.6 \pm 26.6$ & $207.0 \pm 17.6$ & $69.8 \pm 11.4$ & $0.35_{-0.03}^{+0.03}$ & 1.72 & 861.0 & 7.79 & 3.14 & 4.78 & 2.77 & NP \\
\hline 284 & $1237 \quad 18.83$ & +620652.0 & 0.9 & $46.3 \pm 11.7$ & $44.6 \pm 9.6$ & $<18.0$ & $<14.9$ & $<0.41$ & $>1.61$ & 396.1 & 1.06 & 0.51 & $<0.92$ & $<1.31$ & \\
\hline 285 & 123719.03 & +621025.6 & 0.6 & $66.9 \pm 11.2$ & $53.3 \pm 9.2$ & $13.9 \pm 7.2^{\mathrm{b}}$ & $<11.6$ & $0.26_{-0.14}^{+0.15}$ & 1.88 & 872.6 & 0.60 & 0.28 & 0.31 & $<0.44$ & $\mathrm{C}$ \\
\hline 286 & $1237 \quad 19.87$ & +620433.0 & 1.2 & $46.0 \pm 14.7$ & $17.0 \pm 9.2^{\mathrm{bc}}$ & $29.2 \pm 12.6$ & $<27.9$ & $1.75_{-0.97}^{+2.16}$ & 0.26 & 381.0 & 2.28 & 0.19 & 2.11 & $<3.07$ & $\mathrm{O}$ \\
\hline 287 & 123719.90 & +620955.2 & 0.6 & $248.3 \pm 18.8$ & $144.8 \pm 13.9$ & $98.3 \pm 12.8$ & $27.0 \pm 8.2$ & $0.69_{-0.11}^{+0.12}$ & 1.18 & 901.1 & 3.19 & 0.71 & 2.42 & 1.09 & $\mathrm{C}$ \\
\hline 288 & $1237 \quad 20.32$ & +621352.8 & 0.6 & $29.7 \pm 7.9$ & $<6.3$ & $30.2 \pm 7.8$ & $13.0 \pm 5.9$ & $>4.77$ & $<-0.54$ & 767.6 & 1.06 & $<0.04$ & 1.29 & 0.74 & $\mathrm{C}$ \\
\hline 289 & $1237 \quad 20.40$ & +621523.5 & 0.6 & $28.2 \pm 8.1$ & $17.6 \pm 6.2^{\mathrm{a}}$ & $<15.3$ & $<9.6$ & $<0.88$ & $1.40^{\mathrm{d}}$ & 928.4 & 0.31 & 0.08 & $<0.35$ & $<0.36$ & $\mathrm{C}, \mathrm{NP}$ \\
\hline 290 & 123721.64 & +621246.5 & 0.6 & $16.9 \pm 7.0^{\mathrm{b}}$ & $13.4 \pm 5.6$ & $<11.8$ & $<7.8$ & $<0.89$ & $1.40^{\mathrm{d}}$ & 858.3 & 0.20 & 0.07 & $<0.29$ & $<0.31$ & C \\
\hline 291 & 123721.87 & +621035.3 & 0.6 & $53.7 \pm 10.4$ & $<12.4$ & $44.9 \pm 9.6$ & $28.2 \pm 8.1$ & $>3.67$ & $<-0.29$ & 835.5 & 1.57 & $<0.07$ & 1.68 & 1.49 & $\mathrm{C}, \mathrm{NP}$ \\
\hline 292 & 123722.38 & +620536.3 & 1.1 & $73.8 \pm 14.6$ & $25.4 \pm 8.9$ & $43.6 \pm 12.1$ & $<23.5$ & $1.76_{-0.67}^{+1.07}$ & 0.25 & 375.9 & 3.72 & 0.29 & 3.20 & $<2.61$ & \\
\hline 293 & 123722.72 & +620935.2 & 0.7 & $111.8 \pm 14.1$ & $59.6 \pm 10.0$ & $50.0 \pm 10.7$ & $<15.7$ & $0.85_{-0.22}^{+0.25}$ & 0.97 & 864.9 & 1.68 & 0.30 & 1.34 & $<0.68$ & $\mathrm{C}$ \\
\hline 294 & 123723.20 & +621538.5 & 0.6 & $144.3 \pm 14.6$ & $90.8 \pm 11.4$ & $52.7 \pm 9.8$ & $18.2 \pm 6.9$ & $0.58_{-0.13}^{+0.14}$ & 1.33 & 918.5 & 1.67 & 0.44 & 1.23 & 0.69 & $\mathrm{C}$ \\
\hline 295 & $12 \quad 3723.53$ & +622112.7 & 1.0 & $130.0 \pm 17.8$ & $86.1 \pm 12.8$ & $44.9 \pm 13.3$ & $<28.1$ & $0.54_{-0.17}^{+0.19}$ & 1.40 & 708.1 & 1.88 & 0.54 & 1.36 & $<1.46$ & \\
\hline 296 & $12 \quad 3724.02$ & +621304.2 & 0.6 & $168.0 \pm 15.3$ & $47.3 \pm 8.5$ & $125.6 \pm 13.6$ & $52.9 \pm 9.4$ & $2.66_{-0.50}^{+0.65}$ & -0.07 & 739.2 & 5.02 & 0.28 & 4.97 & 2.97 & $\mathrm{C}$ \\
\hline 297 & 123724.17 & +621240.3 & 0.6 & $<15.5$ & $11.3 \pm 5.5$ & $<11.6$ & $<11.2$ & $<1.04$ & $1.40^{\mathrm{d}}$ & 766.8 & $<0.21$ & 0.07 & $<0.32$ & $<0.51$ & $\mathrm{C}$ \\
\hline 298 & $1237 \quad 24.29$ & +621359.5 & 0.6 & $222.3 \pm 17.3$ & $82.3 \pm 10.7$ & $147.2 \pm 14.4$ & $50.3 \pm 9.3$ & $1.78_{-0.27}^{+0.32}$ & 0.24 & 833.1 & 5.09 & 0.43 & 4.80 & 2.39 & $\mathrm{C}$ \\
\hline 299 & 123725.06 & +620856.6 & 0.8 & $197.7 \pm 17.6$ & $94.9 \pm 11.9$ & $101.9 \pm 13.4$ & $32.6 \pm 9.1$ & $1.08_{-0.19}^{+0.21}$ & 0.72 & 824.9 & 3.56 & 0.50 & 3.05 & 1.53 & $\mathrm{C}$ \\
\hline 300 & $12 \quad 3725.47$ & +621941.2 & 0.9 & $<36.0$ & $36.0 \pm 9.5^{\mathrm{a}}$ & $<23.2$ & $<18.9$ & $<0.66$ & $>1.22$ & 857.1 & $<0.48$ & 0.19 & $<0.60$ & $<0.82$ & \\
\hline 301 & 123725.52 & $+62 \quad 1707.6$ & 0.6 & $112.8 \pm 13.8$ & $93.8 \pm 11.7$ & $24.1 \pm 8.4^{\mathrm{b}}$ & $<15.4$ & $0.26_{-0.10}^{+0.10}$ & 1.89 & 887.3 & 0.98 & 0.48 & 0.52 & $<0.58$ & $\mathrm{C}$ \\
\hline 302 & 123725.71 & +621648.6 & 0.6 & $57.9 \pm 10.9$ & $49.9 \pm 9.1$ & $<14.5$ & $<11.8$ & $<0.29$ & $>1.83$ & 889.9 & 0.52 & 0.25 & $<0.32$ & $<0.44$ & $\mathrm{C}$ \\
\hline 303 & 123725.75 & +622243.8 & 1.2 & $334.0 \pm 25.2$ & $210.5 \pm 18.3$ & $124.2 \pm 18.2^{\mathrm{b}}$ & $<33.1$ & $0.61_{-0.10}^{+0.11}$ & 1.29 & 688.2 & 5.29 & 1.35 & 3.99 & $<1.84$ & \\
\hline 304 & 123725.80 & +620230.9 & 1.4 & $136.1 \pm 12.8$ & $114.3 \pm 10.7$ & $25.8 \pm 7.0^{\mathrm{a}}$ & $<13.8$ & $0.24_{-0.07}^{+0.07}$ & 1.94 & 40.5 & 25.39 & 12.45 & 12.22 & $<11.85$ & M \\
\hline 305 & $1237 \quad 26.50$ & +620433.3 & 1.2 & $205.4 \pm 20.6$ & $131.6 \pm 14.7$ & $71.2 \pm 14.9^{\mathrm{c}}$ & $<30.0$ & $0.55_{-0.13}^{+0.14}$ & 1.38 & 380.8 & 5.60 & 1.53 & 4.02 & $<2.89$ & $\mathrm{O}, \mathrm{NP}$ \\
\hline 306 & 123726.60 & +622028.3 & 0.9 & $86.0 \pm 15.4$ & $39.9 \pm 10.1^{\mathrm{b}}$ & $49.7 \pm 12.9^{\mathrm{b}}$ & $<27.4$ & $1.28_{-0.42}^{+0.55}$ & 0.55 & 782.3 & 1.79 & 0.22 & 1.65 & $<1.43$ & \\
\hline 307 & 123726.78 & +621747.6 & 0.7 & $79.1 \pm 12.7$ & $34.3 \pm 8.4$ & $48.0 \pm 10.6$ & $21.6 \pm 8.2^{\mathrm{a}}$ & $1.42_{-0.42}^{+0.56}$ & 0.45 & 885.0 & 1.53 & 0.17 & 1.42 & 0.97 & \\
\hline
\end{tabular}


Table 3-Continued

\begin{tabular}{|c|c|c|c|c|c|c|c|c|c|c|c|c|c|c|c|}
\hline \multirow[b]{2}{*}{$\begin{array}{c}\# \\
(1)\end{array}$} & \multicolumn{2}{|c|}{ Coordinates } & \multirow{2}{*}{$\begin{array}{c}\text { Positional } \\
\text { Error } \\
(4)\end{array}$} & \multicolumn{4}{|c|}{ Counts } & \multirow{2}{*}{$\begin{array}{l}\text { Band } \\
\text { Ratio } \\
(9)\end{array}$} & \multirow{2}{*}{$\begin{array}{c}\text { Effective } \\
\Gamma \\
(10)\end{array}$} & \multirow{2}{*}{$\begin{array}{c}\text { Exposure } \\
\text { Exposure (ks) } \\
(11)\end{array}$} & \multicolumn{4}{|c|}{ Flux } & \multirow[b]{2}{*}{$\begin{array}{l}\text { Notes } \\
(16)\end{array}$} \\
\hline & $\begin{array}{c}\alpha_{2000} \\
(2)\end{array}$ & $\begin{array}{c}\delta_{2000} \\
(3)\end{array}$ & & $\begin{array}{l}\text { FB } \\
(5)\end{array}$ & $\begin{array}{l}\text { SB } \\
(6)\end{array}$ & $\begin{array}{l}\mathrm{HB} \\
(7)\end{array}$ & $\begin{array}{l}\text { UHB } \\
(8)\end{array}$ & & & & $\begin{array}{l}\text { FB } \\
(12)\end{array}$ & $\begin{array}{l}\mathrm{SB} \\
(13)\end{array}$ & $\begin{array}{c}\mathrm{HB} \\
(14)\end{array}$ & $\begin{array}{l}\text { UHB } \\
(15)\end{array}$ & \\
\hline 308 & $\begin{array}{lll}12 & 37 & 29.77\end{array}$ & +621236.7 & 0.6 & $19.8 \pm 8.1$ & $<14.2$ & $<14.1$ & $<11.1$ & - & $1.40^{\mathrm{d}}$ & 781.7 & 0.26 & $<0.08$ & $<0.38$ & $<0.49$ & \\
\hline 309 & 123730.85 & +620849.8 & 0.8 & $402.3 \pm 23.2$ & $228.6 \pm 17.0$ & $161.0 \pm 15.7$ & $44.2 \pm 9.7$ & $0.72_{-0.09}^{+0.09}$ & 1.14 & 374.5 & 12.76 & 2.69 & 9.72 & 4.40 & \\
\hline 310 & 123731.10 & +621030.8 & 0.7 & $34.7 \pm 10.0$ & $20.1 \pm 7.2$ & $<19.8$ & $<16.8$ & $<1.00$ & $1.40^{\mathrm{d}}$ & 832.5 & 0.43 & 0.11 & $<0.51$ & $<0.72$ & \\
\hline 311 & 123731.59 & +621305.2 & 0.6 & $28.7 \pm 9.0$ & $16.8 \pm 6.4$ & $<16.8$ & $<13.9$ & $<1.00$ & $1.40^{\mathrm{d}}$ & 864.7 & 0.34 & 0.09 & $<0.41$ & $<0.55$ & \\
\hline 312 & 123731.74 & +621703.5 & 0.7 & $244.7 \pm 18.9$ & $174.9 \pm 15.3$ & $83.1 \pm 12.3$ & $36.0 \pm 9.2$ & $0.48_{-0.08}^{+0.08}$ & 1.49 & 849.9 & 2.80 & 0.92 & 2.03 & 1.47 & \\
\hline 313 & 123732.19 & +621245.9 & 0.6 & $28.1 \pm 9.0^{\mathrm{a}}$ & $15.8 \pm 6.4$ & $<17.7$ & $<13.8$ & $<1.12$ & $1.40^{\mathrm{d}}$ & 861.3 & 0.33 & 0.08 & $<0.43$ & $<0.55$ & \\
\hline 314 & 123734.08 & $+62 \quad 1623.9$ & 0.7 & $51.9 \pm 11.2$ & $<18.2$ & $38.0 \pm 9.8$ & $<18.8$ & $>2.11$ & $<0.10$ & 864.2 & 1.23 & $<0.09$ & 1.25 & $<0.90$ & \\
\hline 315 & 123734.18 & +620931.4 & 0.8 & $24.3 \pm 9.3^{\mathrm{a}}$ & $19.3 \pm 7.1^{\mathrm{a}}$ & $<16.7$ & $<15.1$ & $<0.88$ & $1.40^{\mathrm{d}}$ & 356.8 & 0.70 & 0.24 & $<1.00$ & $<1.54$ & \\
\hline 316 & 123734.55 & +621355.9 & 0.6 & $221.4 \pm 18.0$ & $157.4 \pm 14.4$ & $65.6 \pm 11.2$ & $<18.1$ & $0.42_{-0.08}^{+0.08}$ & 1.60 & 898.1 & 2.25 & 0.79 & 1.47 & $<0.68$ & \\
\hline 317 & 123734.94 & +621916.1 & 0.9 & $40.2 \pm 13.3^{\mathrm{b}}$ & $41.2 \pm 10.0$ & $<23.9$ & $<19.8$ & $<0.59$ & $>1.31$ & 832.1 & 0.52 & 0.22 & $<0.63$ & $<0.87$ & \\
\hline 318 & 123735.13 & +621307.0 & 0.7 & $34.3 \pm 9.9$ & $<11.7$ & $35.6 \pm 9.4$ & $16.6 \pm 7.4^{\mathrm{b}}$ & $>3.05$ & $<-0.16$ & 876.4 & 0.90 & $<0.06$ & 1.21 & 0.80 & \\
\hline 319 & 123735.45 & +621354.0 & 0.7 & $131.6 \pm 14.7$ & $61.0 \pm 9.9$ & $77.4 \pm 11.9$ & $32.8 \pm 8.8$ & $1.27_{-0.26}^{+0.31}$ & 0.56 & 883.7 & 2.41 & 0.30 & 2.22 & 1.43 & \\
\hline 320 & 123735.96 & +621805.6 & 0.8 & $48.9 \pm 12.4$ & $<22.7$ & $<26.1$ & $<20.9$ & -0 & $1.40^{\mathrm{d}}$ & 793.1 & 0.63 & $<0.13$ & $<0.70$ & $<0.94$ & 1 \\
\hline 321 & 123736.58 & $+62 \quad 1051.2$ & 0.8 & $90.8 \pm 13.3$ & $44.2 \pm 9.2$ & $44.4 \pm 10.4$ & $<17.5$ & $1.02_{-0.30}^{+0.36}$ & 0.79 & 850.8 & 1.53 & 0.23 & 1.27 & $<0.79$ & 5 \\
\hline 322 & 123736.89 & +621428.9 & 0.7 & $432.6 \pm 24.0$ & $226.0 \pm 17.0$ & $206.1 \pm 17.2$ & $89.1 \pm 12.2$ & $0.91_{-0.10}^{+0.11}$ & 0.90 & 882.9 & 6.63 & 1.12 & 5.47 & 3.73 & 1 \\
\hline 323 & 123737.09 & +621834.4 & 0.9 & $259.8 \pm 20.7$ & $133.9 \pm 14.3$ & $117.0 \pm 15.3$ & $35.1 \pm 10.6$ & $0.89_{-0.15}^{+0.16}$ & 0.93 & 871.7 & 3.98 & 0.67 & 3.17 & 1.54 & \\
\hline 324 & 123737.82 & +621630.3 & 0.8 & $19.8 \pm 9.7$ & $27.6 \pm 8.1$ & $<14.6$ & $<14.5$ & $<0.54$ & $1.40^{\mathrm{d}}$ & 815.5 & 0.25 & 0.15 & $<0.38$ & $<0.63$ & \\
\hline 325 & 123738.01 & +621713.5 & 0.8 & $50.1 \pm 12.1$ & $21.6 \pm 8.0^{\mathrm{a}}$ & $29.2 \pm 10.1$ & $<21.0$ & $1.38_{-0.60}^{+0.94}$ & 0.48 & 838.9 & 1.01 & 0.11 & 0.91 & $<1.00$ & \\
\hline 326 & 123738.03 & +621308.8 & 0.7 & $114.1 \pm 14.1$ & $35.5 \pm 8.4$ & $86.2 \pm 12.4$ & $39.1 \pm 9.3$ & $2.44_{-0.59}^{+0.84}$ & -0.01 & 868.3 & 2.82 & 0.18 & 2.87 & 1.88 & \\
\hline 327 & 123739.24 & $+62 \quad 2059.8$ & 1.1 & $3332.6 \pm 63.6$ & $2383.4 \pm 52.2$ & $965.1 \pm 38.3$ & $337.5 \pm 26.1$ & $0.42_{-0.02}^{+0.02}$ & 1.60 & 831.7 & 36.60 & 12.82 & 23.93 & 14.70 & NP \\
\hline 328 & 123739.67 & +621337.3 & 0.7 & $95.0 \pm 13.3$ & $71.2 \pm 10.7$ & $24.4 \pm 9.0$ & $<15.9$ & $0.34_{-0.13}^{+0.14}$ & 1.73 & 886.6 & 0.91 & 0.36 & 0.54 & $<0.60$ & \\
\hline 329 & 123739.74 & +621701.6 & 0.8 & $121.4 \pm 15.3$ & $88.4 \pm 11.9$ & $35.3 \pm 10.6$ & $<18.3$ & $0.41_{-0.13}^{+0.14}$ & 1.62 & 830.7 & 1.32 & 0.48 & 0.86 & $<0.76$ & \\
\hline 330 & 123740.98 & +621200.1 & 0.8 & $1735.5 \pm 44.7$ & $1202.9 \pm 36.7$ & $540.1 \pm 26.9$ & $141.0 \pm 16.3$ & $0.45_{-0.03}^{+0.03}$ & 1.54 & 836.3 & 19.62 & 6.43 & 13.22 & 5.83 & \\
\hline 331 & 123741.15 & +621047.4 & 0.8 & $43.2 \pm 10.9$ & $28.6 \pm 8.0$ & $15.0 \pm 8.3$ & $<15.3$ & $0.53_{-0.32}^{+0.36}$ & 1.41 & 502.3 & 0.87 & 0.25 & 0.63 & $<1.07$ & NP \\
\hline 332 & 123741.39 & $+62 \quad 1251.1$ & 0.7 & $39.0 \pm 10.8$ & $15.1 \pm 7.2$ & $24.2 \pm 9.2^{\mathrm{b}}$ & $<18.8$ & $1.62_{-0.81}^{+1.59}$ & 0.32 & 866.7 & 0.82 & 0.08 & 0.75 & $<0.87$ & \\
\hline 333 & 123742.33 & +621518.2 & 0.8 & $118.8 \pm 14.9$ & $71.7 \pm 10.9$ & $49.3 \pm 11.0$ & $<21.8$ & $0.69_{-0.18}^{+0.20}$ & 1.18 & 874.7 & 1.58 & 0.36 & 1.24 & $<0.90$ & \\
\hline 334 & 123742.64 & $+62 \quad 1812.2$ & 0.9 & $1902.0 \pm 48.1$ & $1389.3 \pm 39.9$ & $520.0 \pm 28.6$ & $178.4 \pm 19.7$ & $0.38_{-0.02}^{+0.02}$ & 1.66 & 839.2 & 19.99 & 7.42 & 12.49 & 7.40 & \\
\hline 335 & 123742.80 & +620752.7 & 1.0 & $22.8 \pm 12.0^{\mathrm{b}}$ & $12.6 \pm 8.1^{\mathrm{a}}$ & $<23.3$ & $<18.5$ & $<1.90$ & $1.40^{\mathrm{d}}$ & 335.1 & 0.70 & 0.17 & $<1.49$ & $<2.02$ & \\
\hline 336 & 123743.62 & +620539.2 & 1.2 & $60.2 \pm 15.7$ & $35.4 \pm 10.1$ & $23.6 \pm 12.9$ & $<26.3$ & $0.69_{-0.41}^{+0.47}$ & 1.18 & 380.9 & 1.83 & 0.41 & 1.40 & $<2.65$ & \\
\hline 337 & 123744.53 & +620641.4 & 1.1 & $<36.1$ & $13.8 \pm 9.2^{\mathrm{a}}$ & $<28.5$ & $<24.5$ & $\begin{array}{l}<2.14 \\
<.41\end{array}$ & $1.40^{\mathrm{d}}$ & 384.4 & $<0.96$ & 0.16 & $<1.60$ & $<2.37$ & \\
\hline 338 & 123744.91 & $+6207 \quad 19.5$ & 1.1 & $358.2 \pm 23.7$ & $220.0 \pm 17.7$ & $128.8 \pm 16.0$ & $45.3 \pm 11.3$ & $0.60_{-0.09}^{+0.09}$ & 1.30 & 352.8 & 11.00 & 2.75 & 8.01 & 4.79 & \\
\hline 339 & 123746.25 & +621035.6 & 0.9 & $74.0 \pm 12.8$ & $34.1 \pm 8.3$ & $38.7 \pm 10.2^{\mathrm{a}}$ & $<20.4$ & $\begin{array}{l}1.15_{-0.38}^{+0.48} \\
\end{array}$ & 0.67 & 386.6 & 2.94 & 0.38 & 2.50 & $<2.06$ & \\
\hline 340 & 123746.74 & +620713.7 & 1.1 & $59.9 \pm 15.0^{\mathrm{a}}$ & $40.1 \pm 10.6$ & $<30.5$ & $<25.7$ & $<0.79$ & $>1.05$ & 346.7 & 2.15 & 0.50 & $<2.04$ & $<2.85$ & \\
\hline 341 & 123748.09 & $+62 \quad 2126.1$ & 1.2 & $100.4 \pm 16.7^{\mathrm{a}}$ & $53.0 \pm 11.2^{\mathrm{b}}$ & $50.0 \pm 13.4^{\mathrm{a}}$ & $<29.3$ & $0.97_{-0.31}^{+0.37}$ & 0.84 & 471.1 & 2.98 & 0.49 & 2.59 & $<2.52$ & \\
\hline 342 & 123750.19 & +621358.7 & 0.9 & $47.9 \pm 12.7$ & $<19.1$ & $42.1 \pm 11.4$ & $28.7 \pm 9.8^{\mathrm{b}}$ & $>2.22$ & $<0.06$ & 856.3 & 1.16 & $<0.10$ & 1.40 & 1.40 & \\
\hline 343 & 123752.70 & +621627.8 & 0.9 & $3412.0 \pm 62.6$ & $2276.5 \pm 50.6$ & $1131.9 \pm 38.3$ & $371.9 \pm 24.7$ & $0.50_{-0.02}^{+0.02}$ & 1.46 & 813.2 & 41.54 & 12.51 & 29.05 & 16.17 & \\
\hline 344 & 123752.71 & $+6207 \quad 20.1$ & 1.2 & $128.4 \pm 17.7$ & $62.8 \pm 11.9$ & $60.3 \pm 13.8$ & $<27.0$ & $0.99_{-0.28}^{+0.32}$ & 0.82 & 331.1 & 5.48 & 0.83 & 4.49 & $<3.31$ & \\
\hline 345 & 123754.28 & +621059.4 & 1.0 & $50.7 \pm 12.4$ & $<15.7$ & $47.6 \pm 11.4$ & $30.3 \pm 9.7$ & $>3.08$ & $<-0.17$ & 392.6 & 2.98 & $<0.17$ & 3.66 & 3.36 & \\
\hline
\end{tabular}


Table 3-Continued

\begin{tabular}{|c|c|c|c|c|c|c|c|c|c|c|c|c|c|c|c|}
\hline \multirow[b]{2}{*}{$\begin{array}{c}\# \\
(1)\end{array}$} & \multicolumn{2}{|c|}{ Coordinates } & \multirow{2}{*}{$\begin{array}{c}\text { Positional } \\
\text { Error } \\
(4)\end{array}$} & \multicolumn{4}{|c|}{ Counts } & \multirow{2}{*}{$\begin{array}{c}\text { Band } \\
\text { Ratio } \\
(9)\end{array}$} & \multirow{2}{*}{$\begin{array}{c}\text { Effective } \\
\Gamma \\
(10)\end{array}$} & \multirow{2}{*}{$\begin{array}{c}\text { Exposure } \\
\text { Exposure (ks) } \\
\quad(11)\end{array}$} & \multirow[b]{2}{*}{$\begin{array}{c}\text { FB } \\
(12)\end{array}$} & \multicolumn{3}{|c|}{ Flux } & \multirow[b]{2}{*}{$\begin{array}{c}\text { Notes } \\
(16)\end{array}$} \\
\hline & $\begin{array}{c}\alpha_{2000} \\
(2)\end{array}$ & $\begin{array}{c}\delta_{2000} \\
(3)\end{array}$ & & $\begin{array}{l}\text { FB } \\
(5)\end{array}$ & $\begin{array}{l}\text { SB } \\
(6)\end{array}$ & $\begin{array}{l}\mathrm{HB} \\
(7)\end{array}$ & $\begin{array}{l}\text { UHB } \\
(8)\end{array}$ & & & & & $\begin{array}{l}\text { SB } \\
(13)\end{array}$ & $\begin{array}{l}\mathrm{HB} \\
(14)\end{array}$ & $\begin{array}{l}\text { UHB } \\
(15)\end{array}$ & \\
\hline 346 & $1237 \quad 54.53$ & +621008.8 & 1.0 & $46.0 \pm 12.6$ & $31.0 \pm 8.7$ & $<23.5$ & $<18.7$ & $<0.77$ & $>1.07$ & 388.9 & 1.46 & 0.35 & $<1.38$ & $<1.80$ & \\
\hline 347 & 123754.61 & +621842.3 & 1.1 & $36.3 \pm 15.7^{\mathrm{b}}$ & $31.0 \pm 10.6$ & $<31.3$ & $<24.9$ & $<1.04$ & $>0.77$ & 827.2 & 0.64 & 0.16 & $<0.93$ & $<1.19$ & \\
\hline 348 & $1237 \quad 57.33$ & +621627.9 & 1.0 & $510.0 \pm 28.0$ & $402.6 \pm 23.4$ & $106.9 \pm 16.3$ & $<28.9$ & $0.27_{-0.04}^{+0.04}$ & 1.87 & 834.3 & 4.78 & 2.19 & 2.46 & $<1.18$ & NP \\
\hline 349 & 123759.63 & $+62 \quad 1102.1$ & 1.0 & $5564.1 \pm 77.6$ & $4100.2 \pm 66.0$ & $1469.4 \pm 42.2$ & $436.7 \pm 25.3$ & $0.36_{-0.01}^{+0.01}$ & 1.70 & 388.3 & 123.90 & 47.51 & 75.37 & 38.81 & NP \\
\hline 350 & 123800.77 & +620839.7 & 1.2 & $55.9 \pm 15.0$ & $<22.8$ & $47.2 \pm 13.3^{\mathrm{c}}$ & $<27.2$ & $>2.13$ & $<0.09$ & 369.0 & 3.10 & $<0.27$ & 3.70 & $<3.24$ & $\mathrm{O}, \mathrm{NP}$ \\
\hline 351 & $\begin{array}{lll}12 & 38 & 00.97\end{array}$ & +621336.1 & 1.0 & $6511.8 \pm 83.8$ & $5280.5 \pm 74.6$ & $1269.1 \pm 39.9$ & $389.9 \pm 24.8$ & $0.24_{-0.01}^{+0.01}$ & 1.93 & 599.8 & 82.38 & 40.15 & 40.12 & 21.69 & \\
\hline 352 & $1238 \quad 03.14$ & +621104.3 & 1.1 & $129.3 \pm 13.9$ & $80.4 \pm 10.2$ & $48.0 \pm 10.1$ & $<24.1$ & $0.61_{-0.14}^{+0.15}$ & 1.30 & 385.0 & 3.64 & 0.92 & 2.70 & $<2.27$ & $\mathrm{M}$ \\
\hline 353 & 123804.47 & +621524.8 & 1.1 & $126.8 \pm 18.3$ & $50.7 \pm 11.6^{\mathrm{b}}$ & $75.1 \pm 15.1$ & $<31.1$ & $1.50_{-0.41}^{+0.54}$ & 0.40 & 814.2 & 2.74 & 0.27 & 2.45 & $<1.57$ & \\
\hline 354 & 123805.25 & +620739.1 & 1.3 & $158.1 \pm 19.3$ & $86.6 \pm 13.6$ & $80.7 \pm 14.9$ & $<31.0$ & $0.96_{-0.22}^{+0.25}$ & 0.85 & 330.2 & 6.64 & 1.15 & 5.96 & $<3.82$ & \\
\hline 355 & 123806.11 & +621046.9 & 1.1 & $114.9 \pm 16.9$ & $37.2 \pm 10.2$ & $74.3 \pm 14.1$ & $25.6 \pm 10.4^{\mathrm{b}}$ & $2.03_{-0.58}^{+0.86}$ & 0.13 & 377.5 & 6.12 & 0.43 & 5.58 & 2.88 & \\
\hline 356 & $12 \quad 38 \quad 06.92$ & +621243.1 & 1.1 & $68.0 \pm 14.2$ & $68.9 \pm 11.6$ & $<24.0$ & $<20.7$ & $<0.35$ & $>1.72$ & 378.2 & 1.53 & 0.82 & $<1.25$ & $<1.86$ & \\
\hline 357 & $1238 \quad 10.57$ & +621728.8 & 1.2 & $353.3 \pm 25.7$ & $48.8 \pm 12.2$ & $308.3 \pm 23.3$ & $124.1 \pm 16.7$ & $6.46_{-1.38}^{+2.21}$ & -0.87 & 695.8 & 15.91 & 0.31 & 16.00 & 8.92 & \\
\hline 358 & 123813.38 & +620858.3 & 1.3 & $116.2 \pm 19.7$ & $47.4 \pm 12.6$ & $66.3 \pm 15.9$ & $<32.9$ & $1.44_{-0.46}^{+0.63}$ & 0.44 & 345.3 & 5.81 & 0.60 & 5.13 & $<4.03$ & I \\
\hline 359 & 123813.46 & $+62 \quad 1134.1$ & 1.2 & $32.9 \pm 14.1$ & $<22.6$ & $15.4 \pm 11.8^{b}$ & $9.0 \pm 9.9^{\mathrm{b}}$ & $\begin{array}{l}-0.46 \\
>0.69\end{array}$ & $<1.17$ & 369.7 & 1.04 & $<0.27$ & 0.93 & 0.90 & $\mapsto$ \\
\hline 360 & $1238 \quad 16.79$ & +621054.4 & 1.3 & $54.6 \pm 16.3^{\mathrm{b}}$ & $52.5 \pm 11.8$ & $<30.1$ & $<24.5$ & $<0.59$ & $>1.33$ & 361.3 & 1.61 & 0.64 & $<1.81$ & $<2.51$ & - \\
\hline 361 & $1238 \quad 17.48$ & +621608.9 & 1.2 & $110.0 \pm 18.8$ & $84.8 \pm 13.7$ & $<34.5$ & $<28.4$ & $<0.42$ & $>1.60$ & 593.7 & 1.69 & 0.64 & $<1.19$ & $<1.72$ & \\
\hline 362 & 123822.38 & $+6214 \quad 16.7$ & 1.3 & $1178.9 \pm 42.8$ & $618.1 \pm 29.7$ & $560.8 \pm 32.3$ & $167.7 \pm 22.5$ & $0.92_{-0.07}^{+0.07}$ & 0.89 & 364.5 & 43.88 & 7.42 & 36.40 & 17.69 & NP \\
\hline 363 & 123823.08 & +621038.9 & 1.3 & $82.3 \pm 19.3$ & $54.4 \pm 12.8$ & $25.1 \pm 15.1$ & $<32.0$ & $0.47_{-0.30}^{+0.32}$ & 1.50 & 329.3 & 2.41 & 0.73 & 1.59 & $<3.55$ & $\mathrm{NP}$ \\
\hline 364 & 123823.50 & +620943.6 & 1.4 & $176.4 \pm 21.4$ & $96.7 \pm 14.6$ & $70.8 \pm 15.8$ & $<32.6$ & $0.75_{-0.19}^{+0.21}$ & 1.10 & 306.3 & 6.98 & 1.40 & 5.30 & $<4.15$ & NP \\
\hline 365 & $1238 \quad 23.83$ & +621517.0 & 1.3 & $70.1 \pm 17.0$ & $27.4 \pm 10.2^{\mathrm{bc}}$ & $39.4 \pm 14.2$ & $<28.4$ & $1.46_{-0.66}^{+1.02}$ & 0.42 & 316.2 & 3.86 & 0.38 & 3.29 & $<3.69$ & $\mathrm{O}$ \\
\hline 366 & $1238 \quad 28.40$ & +620903.3 & 1.5 & $88.8 \pm 14.1$ & $64.9 \pm 10.8$ & $<23.2$ & $<15.7$ & $<0.38$ & $>1.67$ & 56.8 & 13.69 & 5.05 & $<8.32$ & $<10.18$ & \\
\hline 367 & 123831.27 & $+62 \quad 1222.7$ & 1.4 & $88.0 \pm 20.1$ & $51.0 \pm 12.6$ & $<40.7$ & $<31.6$ & $<0.82$ & $>1.01$ & 345.9 & 3.24 & 0.65 & $<2.74$ & $<3.55$ & \\
\hline 368 & 123834.12 & +621437.8 & 1.4 & $68.9 \pm 16.7$ & $18.3 \pm 9.4^{\mathrm{c}}$ & $43.8 \pm 14.1^{\mathrm{a}}$ & $<29.7$ & $2.41_{-1.13}^{+2.64}$ & -0.00 & 219.6 & 6.71 & 0.37 & 5.84 & $<5.96$ & $\mathrm{O}, \mathrm{NP}$ \\
\hline 369 & 123841.03 & +621201.5 & 1.5 & $254.6 \pm 23.8$ & $152.8 \pm 16.4$ & $93.9 \pm 17.0^{\mathrm{a}}$ & $<34.8$ & $0.63_{-013}^{+0.14}$ & 1.26 & 337.0 & 8.36 & 2.01 & 6.16 & $<3.97$ & \\
\hline 370 & 123845.53 & +621000.8 & 1.7 & $219.7 \pm 18.0$ & $175.7 \pm 15.4$ & $35.0 \pm 8.9$ & $<13.0$ & $0.21_{-0.05}^{+0.06}$ & 2.00 & 37.3 & 42.94 & 21.46 & 17.91 & $<12.33$ & \\
\hline
\end{tabular}

${ }^{\mathrm{a}}$ Source was detected with the restricted ACIS grade set with a probability threshold of $10^{-7}$.

${ }^{\mathrm{b}}$ Source was detected with the standard $A S C A$ grade set with a probability threshold of $10^{-5}$

${ }^{\mathrm{c}}$ Source positional offset $>2.5^{\prime \prime}$ from the primary source position.

${ }^{\mathrm{d}}$ Photon index set to given value due to low number of counts. 
$-1-$ 
Table 6. Supplementary Chandra Point-Source Catalog

\begin{tabular}{|c|c|c|c|c|c|c|c|c|c|c|c|c|c|c|c|}
\hline \multirow[b]{2}{*}{$\begin{array}{c}\# \\
(1)\end{array}$} & \multicolumn{2}{|c|}{ Coordinates } & \multirow{2}{*}{$\begin{array}{l}\text { Positional } \\
\text { Error } \\
(4)\end{array}$} & \multicolumn{4}{|c|}{ Counts } & \multirow{2}{*}{$\begin{array}{c}\text { Band } \\
\text { Ratio } \\
(9)\end{array}$} & \multirow{2}{*}{$\begin{array}{c}\text { Effective } \\
\Gamma \\
(10)\end{array}$} & \multirow{2}{*}{$\begin{array}{c}\text { Exposure } \\
\text { Exposure }(\mathrm{ks}) \\
\quad(11)\end{array}$} & \multirow[b]{2}{*}{$\begin{array}{c}\mathrm{FB} \\
(12)\end{array}$} & \multicolumn{3}{|c|}{ Flux } & \multirow[b]{2}{*}{$\begin{array}{c}\text { Notes } \\
(16)\end{array}$} \\
\hline & $\begin{array}{l}\alpha_{2000} \\
(2)\end{array}$ & $\begin{array}{c}\delta_{2000} \\
(3)\end{array}$ & & $\begin{array}{l}\text { FB } \\
(5)\end{array}$ & $\begin{array}{l}\text { SB } \\
(6)\end{array}$ & $\begin{array}{l}\mathrm{HB} \\
(7)\end{array}$ & $\begin{array}{l}\text { UHB } \\
(8)\end{array}$ & & & & & $\begin{array}{l}\mathrm{SB} \\
(13)\end{array}$ & $\begin{array}{l}\mathrm{HB} \\
(14)\end{array}$ & $\begin{array}{l}\text { UHB } \\
(15)\end{array}$ & \\
\hline 1 & 123518.54 & +621553.6 & 1.2 & $43.7 \pm 15.6^{\mathrm{b}}$ & $27.8 \pm 11.1$ & $<29.9$ & $<23.2$ & $<1.12$ & $1.40^{\mathrm{e}}$ & 439.1 & 1.02 & 0.28 & $<1.47$ & $<1.96$ & \\
\hline 2 & $1235 \quad 38.43$ & $\begin{array}{r}+621932.7 \\
\end{array}$ & 1.2 & $65.8 \pm 14.8$ & $36.1 \pm 10.2^{\mathrm{d}}$ & $<30.2$ & $<23.1$ & $<0.86$ & $>0.96$ & 465.5 & 1.85 & 0.34 & $<1.53$ & $<1.92$ & $\mathrm{O}, \mathrm{NP}$ \\
\hline 3 & 123641.68 & $+62 \quad 2437.3$ & 1.3 & $97.0 \pm 19.2$ & $65.7 \pm 13.2$ & $<39.3$ & $<30.8$ & $<0.63$ & $>1.26$ & 552.9 & 1.94 & 0.52 & $<1.60$ & $<2.18$ & NP \\
\hline 4 & 123643.74 & $\begin{array}{r}622554.2 \\
\end{array}$ & 1.4 & $103.1 \pm 23.9$ & $<35.5$ & $92.6 \pm 20.8^{\mathrm{d}}$ & $48.2 \pm 16.6^{\mathrm{d}}$ & $>2.80$ & $<-0.10$ & 554.6 & 4.17 & $<0.28$ & 5.23 & 4.24 & $\mathrm{O}, \mathrm{NP}$ \\
\hline 5 & 123644.91 & +622650.1 & 1.5 & $279.2 \pm 27.6$ & $157.9 \pm 18.2^{\mathrm{d}}$ & $113.9 \pm 21.0^{\mathrm{d}}$ & $<42.7$ & $0.78_{-0.16}^{+0.18}$ & 1.06 & 534.6 & 6.46 & 1.29 & 5.13 & $<3.41$ & $\mathrm{O}$ \\
\hline 6 & 123705.56 & +622456.6 & 1.3 & $93.8 \pm 20.0$ & $36.4 \pm 12.4^{\mathrm{d}}$ & $54.7 \pm 16.6^{\mathrm{d}}$ & $<35.3$ & $1.55_{-0.61}^{+0.93}$ & 0.36 & 419.9 & 4.00 & 0.38 & 3.56 & $<3.68$ & $\mathrm{O}$ \\
\hline 7 & 123738.93 & +622232.3 & 1.2 & $<40.1$ & $40.1 \pm 10.3$ & $<28.5$ & $<23.3$ & $\begin{array}{l}-0.73 \\
<0.61\end{array}$ & $>1.12$ & 339.9 & $<1.41$ & 0.52 & $<1.93$ & $<2.72$ & NP \\
\hline 8 & $1238 \quad 16.27$ & $+62 \quad 1759.2$ & 1.3 & $<44.0$ & $25.7 \pm 10.8$ & $<33.3$ & $<27.5$ & $<1.35$ & $1.40^{\mathrm{e}}$ & 465.1 & $<0.97$ & 0.24 & $<1.55$ & $<2.26$ & NP \\
\hline 9 & $1238 \quad 16.90$ & +620900.9 & 1.3 & $147.6 \pm 20.6$ & $71.9 \pm 13.7$ & $79.4 \pm 16.1$ & $<32.0$ & $1.13_{-0.29}^{+0.35}$ & 0.68 & 324.7 & 6.92 & 0.97 & 6.18 & $<4.06$ & \\
\hline 10 & 123819.93 & +620932.9 & 1.3 & $87.2 \pm 19.4$ & $35.9 \pm 12.6^{\mathrm{d}}$ & $42.6 \pm 15.3^{\mathrm{d}}$ & $<31.3$ & $1.21_{-0.54}^{+0.79}$ & 0.61 & 353.6 & 3.89 & 0.45 & 3.09 & $<3.67$ & $\mathrm{O}$ \\
\hline 11 & 123829.73 & +621016.7 & 1.4 & $<46.6$ & $44.6 \pm 11.9$ & $<34.3$ & $<28.2$ & $<0.79$ & $>1.05$ & 247.0 & $<2.36$ & 0.79 & $<3.22$ & $<4.47$ & NP \\
\hline
\end{tabular}

${ }^{a}$ Source was detected with the restricted ACIS grade set with a probability threshold of $10^{-7}$.

${ }^{\mathrm{b}}$ Source was detected with the standard $A S C A$ grade set with a probability threshold of $10^{-5}$

${ }^{\mathrm{c}}$ Source positional offset $>2.5^{\prime \prime}$ from the primary source position.

${ }^{\mathrm{d}}$ Photon index set to given value due to low number of counts. 